\title{
Diastereoselective Intermolecular Cobalt-Catalyzed Reductive Aldol Reactions of $\alpha, \beta$-Unsaturated Amides With Ketones
}

\author{
Ralph J. R. Lumby, Pekka M. Joensuu, and Hon Wai Lam* \\ School of Chemistry, University of Edinburgh, Joseph Black Building, The King's Buildings, West \\ Mains Road, Edinburgh, EH9 3JJ, United Kingdom
}

\section{Supporting Information}

Contents

General Information

Preparation of $\beta$-Substituted $\alpha, \beta$-Unsaturated Morpholine Amide Substrates

Reductive Aldol Reactions Using N,N-Dimethylacrylamide

Reductive Aldol Reactions Using 4-Acryloylmorpholine

Reductive Aldol Reactions Using $\beta$-Substituted $\alpha, \beta$-Unsaturated Morpholine Amides

Reductive Aldol Reactions Using $\alpha, \beta$-Unsaturated Morpholine Amide 12

Stereochemical Determinations

NMR Spectra

\section{General Information}

All non-aqueous reactions were carried out under a nitrogen atmosphere in oven-dried apparatus. $\mathrm{CH}_{2} \mathrm{Cl}_{2}$ and THF were dried and purified by passage through activated alumina columns using a solvent purification system from www.glasscontour.com 'Petrol' refers to that fraction of light petroleum ether boiling in the range $40-60{ }^{\circ} \mathrm{C}$. Crotonoyl chloride was distilled from $\mathrm{CaH}_{2}$. Commercially available $\mathrm{CoCl}_{2}$ was dried by heating under vacuum until it turned from purple to blue. All other commercially available reagents were used as received. Thin layer chromatography (TLC) was performed on Merck DF-Alufoilien $60 \mathrm{~F}_{254} 0.2 \mathrm{~mm}$ precoated plates. Product spots were visualized by UV light at $254 \mathrm{~nm}$, and subsequently developed using potassium permanganate or ceric ammonium molybdate solution as appropriate. Flash column chromatography was carried out using silica gel 
(Fisher Scientific $60 \AA$ particle size 35-70 micron) employing the method of Still and co-workers. ${ }^{1}$ Melting points were recorded on a Gallenkamp melting point apparatus and are uncorrected. Infra-red spectra were recorded on a Jasco FT/IR-460 Plus instrument as a thin film on sodium chloride plates or as a dilute solution in $\mathrm{CHCl}_{3} .{ }^{1} \mathrm{H}$ NMR spectra were recorded on a Bruker DPX360 (360 MHz) spectrometer or a Bruker ARX250 (250 MHz) spectrometer. Chemical shifts $(\delta)$ are quoted in parts per million (ppm) downfield of tetramethylsilane, using residual protonated solvent as internal standard $\left(\mathrm{CDCl}_{3}\right.$ at $\left.7.27 \mathrm{ppm}\right)$. Abbreviations used in the description of resonances are: $\mathrm{s}$ (singlet), $\mathrm{d}(\mathrm{doublet}), \mathrm{t}$ (triplet), q, (quartet), app (apparent), br (broad). Coupling constants $(J$ ) are quoted to the nearest 0.1 Hz. Proton-decoupled ${ }^{13} \mathrm{C}$ NMR spectra were recorded on a Bruker DPX360 (90.6 MHz) spectrometer or a Bruker ARX250 (62.9 MHz) spectrometer. Chemical shifts $(\delta)$ are quoted in parts per million $(\mathrm{ppm})$ downfield of tetramethylsilane, using deuterated solvent as internal standard $\left(\mathrm{CDCl}_{3}\right.$ at 77.0 ppm). Assignments were made using the DEPT sequence with secondary pulses at $90^{\circ}$ and $135^{\circ}$. High resolution mass spectra were recorded on a Finnigan MAT 900 XLT spectrometer using the electrospray (ES) positive ion mode at the EPSRC National Mass Spectrometry Service Centre, University of Wales Swansea, Stated calculated mass values refer to that of the ion (i.e. the actual species being detected), not that of the neutral parent compound.

\section{Preparation of $\beta$-Substituted $\alpha, \beta$-Unsaturated Morpholine Amide Substrates}

$(E)-1-M o r p h o l i n-4-y l b u t-2-e n-1-o n e$
$(10 a)$${ }^{2}$ Prepared according to a previously

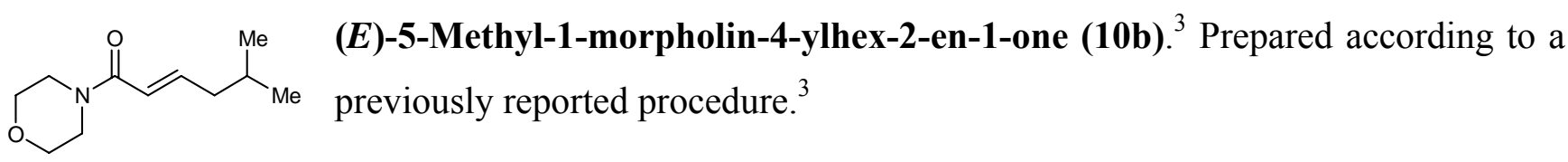<smiles>O=C(/C=C/CCc1ccccc1)N1CCOCC1</smiles>

(E)-1-Morpholin-4-yl-5-phenyl-pent-2-en-1-one (10c). ${ }^{4}$ Prepared according to a previously reported procedure. ${ }^{4}$

1. Still, W. C.; Kahn, M.; Mitra, A. J. Org. Chem. 1978, 43, 2923-2925.

2. Ando, K.; Tsuji, E.; Ando, Y.; Kunitomo, J.-i.; Kobayashi, R.; Yokomizo, T.; Shimizu, T.; Yamashita, M.; Ohta, S.; Nabe, T.; Kohno, S.; Ohishi, Y. Org. Biomol. Chem. 2005, 3, 2129-2139.

3. Janecki, T.; Bodalski, R.; Wieczorek, M.; Bujacz, G. Tetrahedron 1995, 51, 1721-1740.

4. Tosaki, S.-y.; Horiuchi, Y.; Nemoto, T.; Ohshima, T.; Shibasaki, M. Chem. Eur. J. 2004, 10, $1527-1544$. 
(E)-1-Morpholin-4-yl-3-phenylpropenone (10d). ${ }^{5}$ Prepared according to a $\overbrace{\mathrm{O}}^{\mathrm{Ph}}$ previously reported procedure. ${ }^{5}$

(E)-1-Morpholin-4-yl-3-naphthalen-2-ylpropenone (10e)

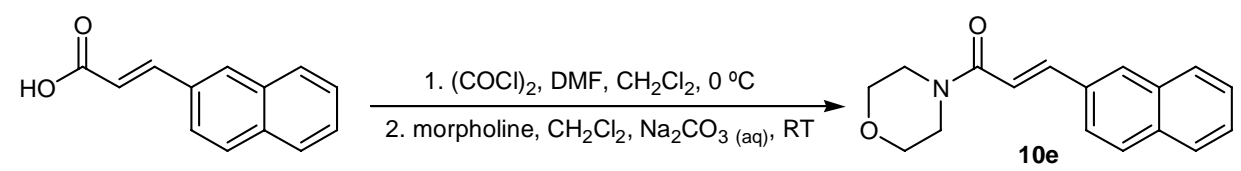

To a solution of (2-naphthyl)acrylic acid (1.49 g, $7.50 \mathrm{mmol})$ and DMF (0.16 mL, $2.06 \mathrm{mmol})$ in $\mathrm{CH}_{2} \mathrm{Cl}_{2}(14 \mathrm{~mL})$ at $0{ }^{\circ} \mathrm{C}$ was added oxalyl chloride $(712 \mu \mathrm{L}, 8.25 \mathrm{mmol})$ dropwise over 2 min and the mixture was stirred at $0{ }^{\circ} \mathrm{C}$ for $1 \mathrm{~h}$. The resulting acid chloride solution was then transferred via cannula to a vigorously stirred mixture of morpholine $(0.44 \mathrm{~mL}, 5.00 \mathrm{mmol})$ in $\mathrm{CH}_{2} \mathrm{Cl}_{2}(5 \mathrm{~mL})$ and saturated aqueous $\mathrm{Na}_{2} \mathrm{CO}_{3}$ solution $(5 \mathrm{~mL})$ and the reaction was stirred for $2 \mathrm{~h}$. The mixture was partitioned between saturated aqueous $\mathrm{NaHCO}_{3}$ solution $(30 \mathrm{~mL})$ and $\mathrm{CH}_{2} \mathrm{Cl}_{2}(30 \mathrm{~mL})$, and the aqueous layer was separated and extracted with $\mathrm{CH}_{2} \mathrm{Cl}_{2}(3 \times 20 \mathrm{~mL})$. The combined organic layers were dried $\left(\mathrm{MgSO}_{4}\right)$ and concentrated in vacuo. Purification of the residue by column chromatography (70\% EtOAc/petrol) gave the $\alpha$, $\beta$-unsaturated morpholine amide $10 \mathrm{e}(1.28 \mathrm{~g}, 96 \%)$ as a white solid. m.p. $146-148{ }^{\circ} \mathrm{C}$; IR $\left(\mathrm{CHCl}_{3}\right)$ 2985, 2860, $1644(\mathrm{C}=\mathrm{O}), 1591(\mathrm{C}=\mathrm{C}), 1442,1269,1115,982,819,727 \mathrm{~cm}^{-1} ;{ }^{1} \mathrm{H}$ NMR $(360$ $\left.\mathrm{MHz} \mathrm{CDCl}_{3}\right) \delta 7.90(1 \mathrm{H}, \mathrm{s}, \operatorname{ArH}), 7.86(1 \mathrm{H}, \mathrm{d}, J=15.4 \mathrm{~Hz}, \operatorname{ArCH}=), 7.85-7.79(3 \mathrm{H}, \mathrm{m}, \operatorname{ArH}), 7.65$ $(1 \mathrm{H}, \mathrm{dd}, J=8.6,1.6 \mathrm{~Hz}, \operatorname{ArH}), 7.50-7.46(2 \mathrm{H}, \mathrm{m}, \mathrm{ArH}), 6.95(1 \mathrm{H}, \mathrm{d}, J=15.4 \mathrm{~Hz}, \mathrm{ArCH}=\mathrm{CH}), 3.72-$ $3.70\left(8 \mathrm{H}, \mathrm{m}, 2\right.$ x $\left.\mathrm{OCH}_{2} \mathrm{CH}_{2} \mathrm{~N}\right) ;{ }^{13} \mathrm{C}$ NMR (62.9 $\left.\mathrm{MHz} \mathrm{CDCl}_{3}\right) \delta 165.4(\mathrm{C}), 143.0(\mathrm{CH}), 133.8(\mathrm{C}), 133.2$ (C), $132.4(\mathrm{C}), 129.1(\mathrm{CH}), 128.4(\mathrm{CH}), 128.3(\mathrm{CH}), 127.6(\mathrm{CH}), 126.8(\mathrm{CH}), 126.5(\mathrm{CH}), 123.4(\mathrm{CH})$, 116.6 (CH), $66.7\left(2 \times \mathrm{CH}_{2}\right), 46.1\left(\mathrm{CH}_{2}\right), 42.3\left(\mathrm{CH}_{2}\right)$; HRMS (ES) Exact mass calcd for $\mathrm{C}_{17} \mathrm{H}_{18} \mathrm{NO}_{2}$ $[\mathrm{M}+\mathrm{H}]^{+}:$268.1332, found: 268.1334 .

\section{(E)-3-Furan-2-yl-1-morpholin-4-ylpropenone (10f)}

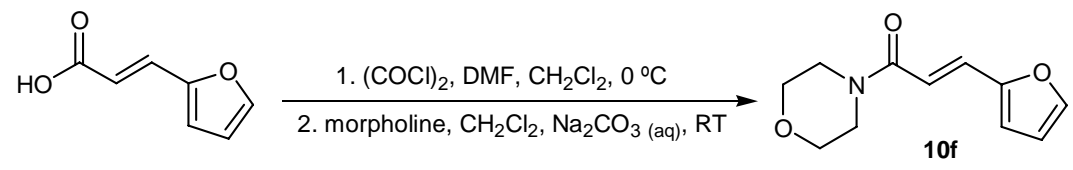

To a solution of (2-furyl)acrylic acid (2.07 g, $15.0 \mathrm{mmol})$ and DMF (0.32 mL, $4.13 \mathrm{mmol})$ in $\mathrm{CH}_{2} \mathrm{Cl}_{2}$ $(27 \mathrm{~mL})$ at $0{ }^{\circ} \mathrm{C}$ was added oxalyl chloride $(1.42 \mathrm{~mL}, 16.5 \mathrm{mmol})$ dropwise over 2 min and the mixture was stirred at $0{ }^{\circ} \mathrm{C}$ for $1 \mathrm{~h}$. The resulting acid chloride solution was then transferred via cannula to a vigorously stirred mixture of morpholine $(0.87 \mathrm{~mL}, 10.0 \mathrm{mmol})$ in $\mathrm{CH}_{2} \mathrm{Cl}_{2}(10 \mathrm{~mL})$ and saturated

5. Badioli, Michele; Ballini, Roberto; Bartolacci, Massimo; Bosica, Giovanna; Torregiani, Elisabetta; Marcantoni, Enrico. J. Org. Chem. 2002, 67, 8938-8942. 
aqueous $\mathrm{Na}_{2} \mathrm{CO}_{3}$ solution $(10 \mathrm{~mL})$ and the reaction was stirred for $4 \mathrm{~h}$. The mixture was partitioned between saturated aqueous $\mathrm{NaHCO}_{3}$ solution $(50 \mathrm{~mL})$ and $\mathrm{CH}_{2} \mathrm{Cl}_{2}(50 \mathrm{~mL})$, and the aqueous layer was separated and extracted with $\mathrm{CH}_{2} \mathrm{Cl}_{2}(3 \times 30 \mathrm{~mL})$. The combined organic layers were dried $\left(\mathrm{MgSO}_{4}\right)$ and concentrated in vacuo. Purification of the residue by column chromatography ( $70 \% \mathrm{EtOAc} /$ petrol) gave the $\alpha, \beta$-unsaturated morpholine amide $10 f(1.96 \mathrm{~g}, 95 \%)$ as a white solid. m.p. $106-108{ }^{\circ} \mathrm{C}$; IR $\left(\mathrm{CHCl}_{3}\right)$ 2963, 2864, $1653(\mathrm{C}=\mathrm{O}), 1458,1362,1258,1113,962,811,739 \mathrm{~cm}^{-1} ;{ }^{1} \mathrm{H}$ NMR $(360 \mathrm{MHz}$, $\left.\mathrm{CDCl}_{3}\right) \delta 7.44(1 \mathrm{H}, \mathrm{d}, J=15.1 \mathrm{~Hz}, \mathrm{O}=\mathrm{CCH}=\mathrm{CH}), 7.41-7.40(1 \mathrm{H}, \mathrm{m}, \mathrm{CH}), 6.73(1 \mathrm{H}, \mathrm{d}, J=15.1 \mathrm{~Hz}$, $\mathrm{O}=\mathrm{CCH}), 6.52(1 \mathrm{H}, \mathrm{d}, J=3.4 \mathrm{~Hz}, \mathrm{CH}), 6.42(1 \mathrm{H}, \mathrm{dd}, J=3.4,1.8 \mathrm{~Hz}, \mathrm{CH}), 3.86-3.48(8 \mathrm{H}, \mathrm{m}, 2 \mathrm{x}$ $\left.\mathrm{OCH}_{2} \mathrm{CH}_{2} \mathrm{~N}\right) ;{ }^{13} \mathrm{C}$ NMR $\left(62.9 \mathrm{MHz} \mathrm{CDCl}_{3}\right) \delta 165.2(\mathrm{C}), 151.4(\mathrm{C}), 143.8(\mathrm{CH}), 129.8(\mathrm{CH}), 113.9(2 \mathrm{x}$ $\mathrm{CH}), 112.1(\mathrm{CH}), 66.7\left(2 \times \mathrm{CH}_{2}\right), 46.1\left(\mathrm{CH}_{2}\right), 42.5\left(\mathrm{CH}_{2}\right)$; HRMS (ES) Exact mass calcd for $\mathrm{C}_{11} \mathrm{H}_{14} \mathrm{NO}_{3}[\mathrm{M}+\mathrm{H}]^{+}: 208.0968$, found: 208.0972.

$\left(\right.$ E)-4-Morpholin-4-yl-4-oxobut-2-enoic acid ethyl ester (12). ${ }^{6}$ Prepared
$\mathrm{CO}_{2} \mathrm{Et}$

Reductive Aldol Reactions Using N,N-Dimethylacrylamide: General Procedure A

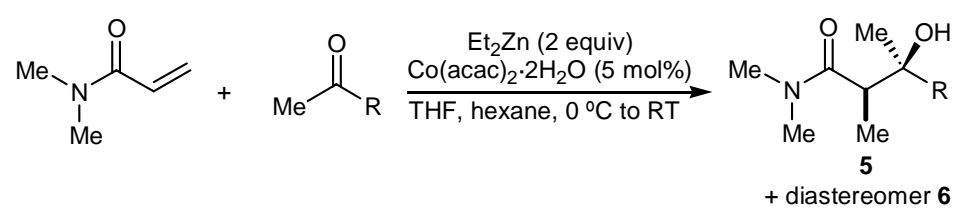

To a solution $\mathrm{Co}(\mathrm{acac})_{2} \cdot 2 \mathrm{H}_{2} \mathrm{O}(12.9 \mathrm{mg}, 0.05 \mathrm{mmol}), N, N$-dimethylacrylamide $(103 \mu \mathrm{L}, 1.00 \mathrm{mmol})$ and the appropriate ketone $(1.10 \mathrm{mmol})$ in THF $(10 \mathrm{~mL})$ at $0{ }^{\circ} \mathrm{C}$ was added $\mathrm{Et}_{2} \mathrm{Zn}(1 \mathrm{M}$ solution in hexane, $2.0 \mathrm{~mL}, 2.0 \mathrm{mmol}$ ) dropwise over $1 \mathrm{~min}$. The reaction was stirred at $0{ }^{\circ} \mathrm{C}$ until complete consumption of the acrylamide as observed by TLC analysis. The reaction was quenched carefully with saturated aqueous $\mathrm{NH}_{4} \mathrm{Cl}$ solution ( $3 \mathrm{~mL}$ ) and the resulting mixture was stirred for 15 minutes. Further saturated aqueous $\mathrm{NH}_{4} \mathrm{Cl}$ solution $\left(30 \mathrm{~mL}\right.$ ) was added, and the mixture was then extracted with $\mathrm{CH}_{2} \mathrm{Cl}_{2}$ $(3 \times 30 \mathrm{~mL})$. The combined organic layers were dried $\left(\mathrm{MgSO}_{4}\right)$ and concentrated in vacuo. Purification of the residue by column chromatography (EtOAc/petrol) afforded the aldol product(s).

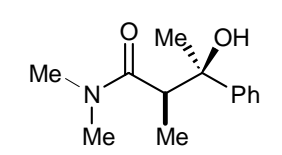

( \pm )-(2R,3R)-3-Hydroxy- $N, N, 2$-trimethyl-3-phenylbutanamide

(5a). ${ }^{7}$ The title compound was prepared according to General Procedure A from acetophenone (127 $\mu \mathrm{L}, 1.10 \mathrm{mmol})$ for a reaction time of $2 \mathrm{~h}$ and purified by column chromatography ( $20 \%$ EtOAc/petrol)

6. Campbell, P. G.; Sumrell, G.; Schramm, C. H. J. Org. Chem. 1961, 26, 697-700.

7. Taniguchi, M.; Hideaki, F.; Koichiro, O.; Kiitiro, U. Tetrahedron Lett. 1992, 33, 4353-4356. 
to give a white solid (166 mg, 75\%) that displayed spectral data consistent with those reported previously. $^{7}$

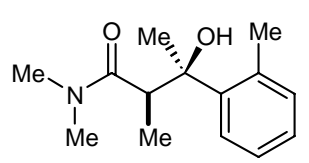

( \pm )-(2R,3R)-3-Hydroxy- $N, N, 2-$ trimethyl-3-(2-methylphenyl)butanamide

(5b). The title compound was prepared according to General Procedure A from 2'methylacetophenone $(144 \mu \mathrm{L}, 1.10 \mathrm{mmol})$ for a reaction time of $4 \mathrm{~h}$ and purified by column chromatography (20\% EtOAc/petrol) to give a white solid (157 mg, 68\%). m.p. $61-63{ }^{\circ} \mathrm{C}$; IR $\left(\mathrm{CHCl}_{3}\right) 3324(\mathrm{OH}), 2973,2934,1619(\mathrm{C}=\mathrm{O}), 1463,1415,1399,1059,765,729 \mathrm{~cm}^{-1} ;{ }^{1} \mathrm{H}$ NMR $(360$ $\left.\mathrm{MHz}, \mathrm{CDCl}_{3}\right) \delta 7.73(1 \mathrm{H}, \mathrm{d}, J=7.7 \mathrm{~Hz}, \mathrm{ArH}), 7.23-7.11(3 \mathrm{H}, \mathrm{m}, \operatorname{ArH}), 6.01(1 \mathrm{H}$, br s, OH), $3.38(1 \mathrm{H}$, q, $\left.J=7.0 \mathrm{~Hz}, \mathrm{CH}_{3} \mathrm{CH}\right), 3.17\left(3 \mathrm{H}, \mathrm{s}, \mathrm{NCH}_{3}\right), 3.05\left(3 \mathrm{H}, \mathrm{s}, \mathrm{NCH}_{3}\right), 2.53\left(3 \mathrm{H}, \mathrm{s}, \mathrm{ArCH}_{3}\right), 1.61(3 \mathrm{H}, \mathrm{s}$, $\left.\mathrm{CH}_{3} \mathrm{COH}\right), 0.94\left(3 \mathrm{H}, \mathrm{d}, J=7.0 \mathrm{~Hz}, \mathrm{CH}_{3} \mathrm{CH}\right) ;{ }^{13} \mathrm{C} \mathrm{NMR}\left(62.9 \mathrm{MHz} \mathrm{CDCl}_{3}\right) \delta 177.7$ (C), $143.4(\mathrm{C})$, $133.4(\mathrm{C}), 132.3(\mathrm{CH}), 127.1(\mathrm{CH}), 126.6(\mathrm{CH}), 125.8(\mathrm{CH}), 75.7(\mathrm{C}), 41.1(\mathrm{CH}), 37.6\left(\mathrm{CH}_{3}\right), 35.6$ $\left(\mathrm{CH}_{3}\right), 28.9\left(\mathrm{CH}_{3}\right), 22.8\left(\mathrm{CH}_{3}\right), 12.9\left(\mathrm{CH}_{3}\right)$; HRMS (ES) Exact mass calcd for $\mathrm{C}_{14} \mathrm{H}_{21} \mathrm{NO}_{2} \mathrm{Na}[\mathrm{M}+\mathrm{Na}]^{+}$: 258.1465, found: 258.1466 .

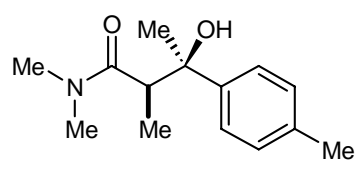

( \pm )-(2R,3R)-3-Hydroxy- $N, N, 2$-trimethyl-3-(4-methylphenyl)butanamide (5c). The title compound was prepared according to General Procedure A from

4'-methylacetophenone $(147 \mu \mathrm{L}, 1.10 \mathrm{mmol})$ for a reaction time of $4 \mathrm{~h}$ and purified by column chromatography (20\% EtOAc/petrol) to give a white solid (186 mg, 79\%). m.p. 112-114 ${ }^{\circ} \mathrm{C}$; IR $\left(\mathrm{CHCl}_{3}\right) 3336(\mathrm{OH}), 2973,2932,1619(\mathrm{C}=\mathrm{O}), 1513,1461,1415,1072,821 \mathrm{~cm}^{-1} ;{ }^{1} \mathrm{H}$ NMR $\left(360 \mathrm{MHz}, \mathrm{CDCl}_{3}\right) \delta 7.35(2 \mathrm{H}, \mathrm{d}, J=8.1 \mathrm{~Hz}, \operatorname{ArH}), 7.16(2 \mathrm{H}, \mathrm{d}, J=8.1 \mathrm{~Hz}, \operatorname{ArH}), 5.87(1 \mathrm{H}, \mathrm{br}$ s, OH), $3.15\left(3 \mathrm{H}, \mathrm{s}, \mathrm{NCH}_{3}\right), 3.05\left(3 \mathrm{H}, \mathrm{s}, \mathrm{NCH}_{3}\right), 2.99\left(1 \mathrm{H}, \mathrm{q}, J=7.1 \mathrm{~Hz}, \mathrm{CH}_{3} \mathrm{CH}\right), 2.35(3 \mathrm{H}, \mathrm{s}$, $\left.\mathrm{ArCH}_{3}\right), 1.51\left(3 \mathrm{H}, \mathrm{s}, \mathrm{CH}_{3} \mathrm{COH}\right), 0.89\left(3 \mathrm{H}, \mathrm{d}, J=7.1 \mathrm{~Hz}, \mathrm{CH}_{3} \mathrm{CH}\right) ;{ }^{13} \mathrm{C} \mathrm{NMR}\left(62.9 \mathrm{MHz} \mathrm{CDCl}_{3}\right) \delta$ $177.8(\mathrm{C}), 143.0(\mathrm{C}), 135.9(\mathrm{C}), 128.7$ (2 x CH), 124.8 (2 x CH), $74.6(\mathrm{C}), 43.8(\mathrm{CH}), 37.7\left(\mathrm{CH}_{3}\right), 35.5$ $\left(\mathrm{CH}_{3}\right), 30.1\left(\mathrm{CH}_{3}\right), 21.0\left(\mathrm{CH}_{3}\right), 12.6\left(\mathrm{CH}_{3}\right)$; HRMS (ES) Exact mass calcd for $\mathrm{C}_{14} \mathrm{H}_{22} \mathrm{NO}_{2}[\mathrm{M}+\mathrm{H}]^{+}$: 236.1645, found: 236.1646 .

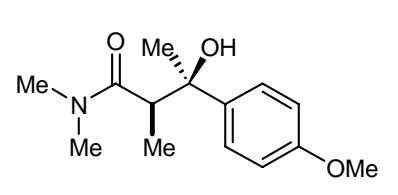

( \pm )-(2R,3R)-3-Hydroxy-3-(4-methoxyphenyl)- $N, N, 2$-trimethylbutanamide (5d). The title compound was prepared according to General Procedure A from 4'-methoxyacetophenone $(165 \mathrm{mg}, 1.10 \mathrm{mmol})$ for a reaction time of $3 \mathrm{~h}$ and purified by column chromatography (20\% EtOAc/petrol) to give a white solid (212 mg, 84\%). m.p. 108-110 ${ }^{\circ} \mathrm{C}$; IR $\left(\mathrm{CHCl}_{3}\right) 3336(\mathrm{OH}), 2971,2934,1616(\mathrm{C}=\mathrm{O}), 1513,1461,1416,1397,1247,1176 \mathrm{~cm}^{-}$ 
${ }^{1} ;{ }^{1} \mathrm{H}$ NMR $\left(360 \mathrm{MHz}, \mathrm{CDCl}_{3}\right) \delta 7.39(2 \mathrm{H}, \mathrm{dm}, J=9.0 \mathrm{~Hz}, \mathrm{ArH}), 6.89(2 \mathrm{H}, \mathrm{dm}, J=9.0 \mathrm{~Hz}, \mathrm{ArH}), 5.87$ $\left(1 \mathrm{H}\right.$, br s, OH), $3.82\left(3 \mathrm{H}, \mathrm{s}, \mathrm{OCH}_{3}\right) 3.15\left(3 \mathrm{H}, \mathrm{s}, \mathrm{NCH}_{3}\right), 3.04\left(3 \mathrm{H}, \mathrm{s}, \mathrm{NCH}_{3}\right), 2.96(1 \mathrm{H}, \mathrm{q}, J=7.1 \mathrm{~Hz}$, $\left.\mathrm{CH}_{3} \mathrm{CH}\right), 1.51\left(3 \mathrm{H}, \mathrm{s}, \mathrm{CH}_{3} \mathrm{COH}\right), 0.89\left(3 \mathrm{H}, \mathrm{d}, J=7.1 \mathrm{~Hz}, \mathrm{CH}_{3} \mathrm{CH}\right) ;{ }^{13} \mathrm{C} \mathrm{NMR}\left(62.9 \mathrm{MHz} \mathrm{CDCl}_{3}\right) \delta$ 177.8 (C), $158.1(\mathrm{C}), 138.2(\mathrm{C}), 126.0(2 \times \mathrm{CH}), 113.3(2 \times \mathrm{CH}), 74.5(\mathrm{C}), 55.2\left(\mathrm{CH}_{3}\right), 43.9(\mathrm{CH}), 37.7$ $\left(\mathrm{CH}_{3}\right), 35.5\left(\mathrm{CH}_{3}\right), 30.0\left(\mathrm{CH}_{3}\right), 12.6\left(\mathrm{CH}_{3}\right)$; HRMS (ES) Exact mass calcd for $\mathrm{C}_{14} \mathrm{H}_{22} \mathrm{NO}_{3}[\mathrm{M}+\mathrm{H}]^{+}$: 252.1594, found: 252.1594 .

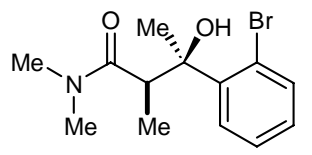

( \pm )-(2R,3R)-3-(2-Bromophenyl)-3-hydroxy- $N, N, 2-t$ rimethylbutanamide

(5e).

The title compound was prepared according to General Procedure A from 2'bromoacetophenone $(148 \mu \mathrm{L}, 1.10 \mathrm{mmol})$ for a reaction time of $29 \mathrm{~h}$ and purified by column chromatography (10\% EtOAc/petrol) to give an off-white solid (169 mg, 56\%). m.p. 74-76 ${ }^{\circ} \mathrm{C}$; IR $\left(\mathrm{CHCl}_{3}\right) 3313(\mathrm{OH}), 2972,2934,1619(\mathrm{C}=\mathrm{O}), 1460,1419,1399,1313,1015,758 \mathrm{~cm}^{-1} ;{ }^{1} \mathrm{H}$ NMR (360 MHz, $\left.\mathrm{CDCl}_{3}\right) \delta$ 8.08-8.05 (1H, m, ArH), 7.58-7.55 (1H, m, ArH), 7.37-7.33 (1H, m, ArH), 7.13-7.08 (1H, m, ArH), 6.32 (1H, br s, OH), $4.21\left(1 \mathrm{H}, \mathrm{q}, J=7.0 \mathrm{~Hz}, \mathrm{CH}_{3} \mathrm{CH}\right), 3.23\left(3 \mathrm{H}, \mathrm{s}, \mathrm{NCH}_{3}\right)$, $3.06\left(3 \mathrm{H}, \mathrm{s}, \mathrm{NCH}_{3}\right), 1.73\left(3 \mathrm{H}, \mathrm{s}, \mathrm{CH}_{3} \mathrm{COH}\right), 0.86\left(3 \mathrm{H}, \mathrm{d}, J=7.0 \mathrm{~Hz}, \mathrm{CH}_{3} \mathrm{CH}\right) ;{ }^{13} \mathrm{C} \mathrm{NMR}(62.9 \mathrm{MHz}$ $\left.\mathrm{CDCl}_{3}\right) \delta 177.5(\mathrm{C}), 143.7(\mathrm{C}), 134.5(\mathrm{CH}), 129.7(\mathrm{CH}), 128.4(\mathrm{CH}), 127.4(\mathrm{CH}), 118.7(\mathrm{C}), 75.3(\mathrm{C})$, $38.3(\mathrm{CH}), 37.6\left(\mathrm{CH}_{3}\right), 35.5\left(\mathrm{CH}_{3}\right), 26.9\left(\mathrm{CH}_{3}\right), 12.7\left(\mathrm{CH}_{3}\right)$; HRMS (ES) Exact mass calcd for $\mathrm{C}_{13} \mathrm{H}_{18} \mathrm{BrNO}_{2}[\mathrm{M}+\mathrm{H}]^{+}: 300.0594$, found: 300.0594 .

$( \pm)-(2 R, 3 R)-3-(4-B r o m o p h e n y l)-3-h y d r o x y-N, N, 2-t r i m e t h y l b u t a n a m i d e ~(5 f)$ and $( \pm)-(2 R, 3 S)-3-(4-$ bromophenyl)-3-hydroxy- $N, N, 2$-trimethylbutanamide (6f)
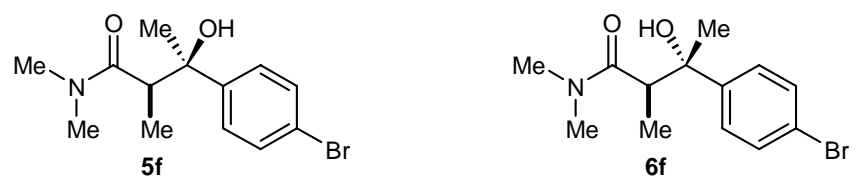

General Procedure A was followed using 4'-bromoacetophenone (219 mg, $1.10 \mathrm{mmol}$ ) for a reaction time of $22 \mathrm{~h}$ and the reaction mixture was purified by column chromatography (20\% EtOAc/petrol) to give the aldol product $\mathbf{5 f}(207 \mathrm{mg}, 73 \%)$ as an off-white solid followed by the aldol product $\mathbf{6 f}$ (45 $\mathrm{mg}$, $15 \%)$ as an off-white solid.

Data for 5f: m.p. 98-100 ${ }^{\circ} \mathrm{C}$; IR $\left(\mathrm{CHCl}_{3}\right) 3335(\mathrm{OH}), 2973,2933,1619(\mathrm{C}=\mathrm{O}), 1489,1461,1415,1399$, 1074, $1009 \mathrm{~cm}^{-1} ;{ }^{1} \mathrm{H}$ NMR $\left(360 \mathrm{MHz}, \mathrm{CDCl}_{3}\right) \delta 7.48(2 \mathrm{H}, \mathrm{d}, J=8.7 \mathrm{~Hz}, \operatorname{ArH}), 7.35(2 \mathrm{H}, \mathrm{d}, J=8.7 \mathrm{~Hz}$, $\operatorname{ArH}), 5.97\left(1 \mathrm{H}\right.$, br s, OH), $3.15\left(3 \mathrm{H}, \mathrm{s}, \mathrm{NCH}_{3}\right), 3.05\left(3 \mathrm{H}, \mathrm{s}, \mathrm{NCH}_{3}\right), 2.95\left(1 \mathrm{H}, \mathrm{q}, J=7.0 \mathrm{~Hz}, \mathrm{CH}_{3} \mathrm{CH}\right)$, $1.50\left(3 \mathrm{H}, \mathrm{s}, \mathrm{CH}_{3} \mathrm{COH}\right), 0.87\left(3 \mathrm{H}, \mathrm{d}, J=7.0 \mathrm{~Hz}, \mathrm{CH}_{3} \mathrm{CH}\right) ;{ }^{13} \mathrm{C} \mathrm{NMR}\left(62.9 \mathrm{MHz} \mathrm{CDCl}_{3}\right) \delta 177.4(\mathrm{C})$, $145.1(\mathrm{C}), 131.1(\mathrm{C}), 126.9(2 \times \mathrm{CH}), 120.4(2 \times \mathrm{CH}), 74.5(\mathrm{CH}), 43.6(\mathrm{CH}), 37.7\left(\mathrm{CH}_{3}\right), 35.6\left(\mathrm{CH}_{3}\right)$, 
$29.8\left(\mathrm{CH}_{3}\right), 12.5\left(\mathrm{CH}_{3}\right)$; HRMS (ES) Exact mass calcd for $\mathrm{C}_{13} \mathrm{H}_{19} \mathrm{BrNO}_{2}[\mathrm{M}+\mathrm{H}]^{+}:$300.0594, found: 300.0593 .

Data for 6f: m.p. $65-67{ }^{\circ} \mathrm{C}$; IR $\left(\mathrm{CHCl}_{3}\right) 3337(\mathrm{OH}), 2936,1617(\mathrm{C}=\mathrm{O}), 1483,1394,1306,1089,1008$, 930, $823 \mathrm{~cm}^{-1} ;{ }^{1} \mathrm{H}$ NMR $\left(360 \mathrm{MHz}, \mathrm{CDCl}_{3}\right) \delta 7.41(2 \mathrm{H}, \mathrm{d}, J=8.7 \mathrm{~Hz}, \mathrm{ArH}), 7.28(2 \mathrm{H}, \mathrm{d}, J=8.7 \mathrm{~Hz}$, $\operatorname{ArH}), 6.10(1 \mathrm{H}, \mathrm{s}, \mathrm{OH}), 3.13\left(1 \mathrm{H}, \mathrm{q}, J=7.0 \mathrm{~Hz}, \mathrm{CH}_{3} \mathrm{CH}\right), 2.86\left(3 \mathrm{H}, \mathrm{s}, \mathrm{NCH}_{3}\right), 2.67\left(3 \mathrm{H}, \mathrm{s}, \mathrm{NCH}_{3}\right)$, $1.42\left(3 \mathrm{H}, \mathrm{s}, \mathrm{CH}_{3} \mathrm{COH}\right), 1.34\left(3 \mathrm{H}, \mathrm{d}, J=7.0 \mathrm{~Hz}, \mathrm{CH}_{3} \mathrm{CH}\right) ;{ }^{13} \mathrm{C} \mathrm{NMR}\left(62.9 \mathrm{MHz}_{\mathrm{CDCl}}\right) \delta 176.8(\mathrm{C})$, $148.1(\mathrm{C}), 131.0(2 \times \mathrm{CH}), 126.4(2 \times \mathrm{CH}), 120.3(\mathrm{C}), 74.6(\mathrm{C}), 43.0(\mathrm{CH}), 37.2\left(\mathrm{CH}_{3}\right), 35.1\left(\mathrm{CH}_{3}\right)$, $27.2\left(\mathrm{CH}_{3}\right), 12.1\left(\mathrm{CH}_{3}\right)$; HRMS (ES) Exact mass calcd for $\mathrm{C}_{13} \mathrm{H}_{19} \mathrm{BrNO}_{2}[\mathrm{M}+\mathrm{H}]^{+}:$300.0594, found: 300.0592 .

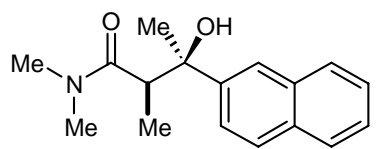

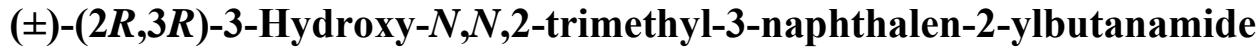
(5g). The title compound was prepared according to General Procedure A from 2-acetonaphthone $(187 \mathrm{mg}, 1.10 \mathrm{mmol})$ for a reaction time of $3 \mathrm{~h}$ and purified by column chromatography (20\% EtOAc/petrol) to give a white solid (212 mg, $78 \%$ ). m.p. $112-114{ }^{\circ} \mathrm{C}$; IR $\left(\mathrm{CHCl}_{3}\right) 3334(\mathrm{OH}), 2973,2933,1618(\mathrm{C}=\mathrm{O}), 1457,1416,1398,1182,1129,748 \mathrm{~cm}^{-1}$; ${ }^{1} \mathrm{H}$ NMR $\left(360 \mathrm{MHz}, \mathrm{CDCl}_{3}\right) \delta 8.05(1 \mathrm{H}, \mathrm{s}, \mathrm{ArH}), 7.90-7.87$ (1H, m, ArH), 7.85-7.82 (2H, m, ArH), 7.51-7.46 $(3 \mathrm{H}, \mathrm{m}, \mathrm{ArH}), 6.08(1 \mathrm{H}$, br s, OH $), 3.20\left(3 \mathrm{H}, \mathrm{s}, \mathrm{NCH}_{3}\right), 3.14\left(1 \mathrm{H}, \mathrm{q}, J=7.1 \mathrm{~Hz}, \mathrm{CH}_{3} \mathrm{CH}\right), 3.08(3 \mathrm{H}, \mathrm{s}$, $\left.\mathrm{NCH}_{3}\right), 1.61\left(3 \mathrm{H}, \mathrm{s}, \mathrm{CH}_{3} \mathrm{COH}\right), 0.90\left(3 \mathrm{H}, \mathrm{d}, J=7.1 \mathrm{~Hz}, \mathrm{CH}_{3} \mathrm{CH}\right) ;{ }^{13} \mathrm{C} \mathrm{NMR}\left(62.9 \mathrm{MHz} \mathrm{CDCl}_{3}\right) \delta$ $177.6(\mathrm{C}), 143.2(\mathrm{C}), 133.2(\mathrm{C}), 132.2(\mathrm{C}), 128.2(\mathrm{CH}), 127.7(\mathrm{CH}), 127.4(\mathrm{CH}), 125.9(\mathrm{CH}), 125.6$ $(\mathrm{CH}), 124.0(\mathrm{CH}), 123.1(\mathrm{CH}), 74.9(\mathrm{C}) 43.5(\mathrm{CH}), 37.7\left(\mathrm{CH}_{3}\right), 35.6\left(\mathrm{CH}_{3}\right), 30.0\left(\mathrm{CH}_{3}\right), 12.7\left(\mathrm{CH}_{3}\right)$; HRMS (ES) Exact mass calcd for $\mathrm{C}_{17} \mathrm{H}_{22} \mathrm{NO}_{2}[\mathrm{M}+\mathrm{H}]^{+}:$272.1645, found: 272.1646.

$( \pm)-(2 R, 3 R)-3-F u r a n-2-y l-3-h y d r o x y-N, N, 2-t r i m e t h y l b u t a n a m i d e ~(5 h)$ and $( \pm)-(2 R, 3 S)-3-f u r a n-2-$ yl-3-hydroxy- $N, N, 2$-trimethylbutanamide $(6 \mathrm{~h})$
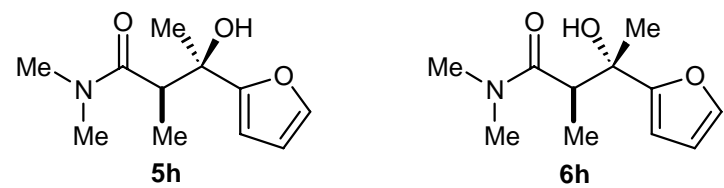

General Procedure A was followed using 2-furyl methyl ketone (121 mg, $1.10 \mathrm{mmol}$ ) for a reaction time of $1 \mathrm{~h}$ and the reaction mixture was purified by column chromatography (20\% EtOAc/petrol) to give aldol product $\mathbf{5 h}(130 \mathrm{mg}, 66 \%)$ as a white solid followed by aldol product $\mathbf{6 h}$ (49 $\mathrm{mg}, 25 \%$ ) as a colorless oil.

Data for 5h: m.p. 78-80 ${ }^{\circ} \mathrm{C}$; IR $\left(\mathrm{CHCl}_{3}\right) 3348(\mathrm{OH}), 2978,2935,1622(\mathrm{C}=\mathrm{O}), 1461,1399,1154,1076$, 1002, $736 \mathrm{~cm}^{-1} ;{ }^{1} \mathrm{H}$ NMR (360 MHz, $\left.\mathrm{CDCl}_{3}\right) \delta$ 7.33-7.32 (1H, m, CH), 6.35-6.34 (2H, m, 2 x CH), 
5.78 (1H, br s, OH), $3.14\left(3 \mathrm{H}, \mathrm{s}, \mathrm{NCH}_{3}\right), 3.11\left(1 \mathrm{H}, \mathrm{q}, J=7.1 \mathrm{~Hz}, \mathrm{CH}_{3} \mathrm{CH}\right), 3.03\left(3 \mathrm{H}, \mathrm{s}, \mathrm{NCH}_{3}\right), 1.52$ $\left(3 \mathrm{H}, \mathrm{s}, \mathrm{CH} \mathbf{H}_{3} \mathrm{COH}\right), 0.98\left(3 \mathrm{H}, \mathrm{d}, J=7.1 \mathrm{~Hz}, \mathrm{CH}_{3} \mathrm{CH}\right) ;{ }^{13} \mathrm{C} \mathrm{NMR}\left(62.9 \mathrm{MHz}^{\mathrm{CDCl}}{ }_{3}\right) \delta 177.2(\mathrm{C}), 157.8$ (C), $140.8(\mathrm{CH}), 110.1(\mathrm{CH}), 105.2(\mathrm{CH}), 73.4(\mathrm{C}), 41.6(\mathrm{CH}), 37.6\left(\mathrm{CH}_{3}\right), 35.3\left(\mathrm{CH}_{3}\right), 27.8\left(\mathrm{CH}_{3}\right)$, 12.7 $\left(\mathrm{CH}_{3}\right)$; HRMS (ES) Exact mass calcd for $\mathrm{C}_{11} \mathrm{H}_{17} \mathrm{NO}_{3} \mathrm{Na}[\mathrm{M}+\mathrm{Na}]^{+}:$234.1101, found: 234.1103 .

Data for 6h: IR (film) $3409(\mathrm{OH}), 2989,2938,1619(\mathrm{C}=\mathrm{O}), 1505,1400,1154,1069,938,737 \mathrm{~cm}^{-1} ;{ }^{1} \mathrm{H}$ NMR $\left(360 \mathrm{MHz}, \mathrm{CDCl}_{3}\right) \delta 7.27(1 \mathrm{H}, \mathrm{s}, \mathrm{ArH}), 6.28(1 \mathrm{H}, \mathrm{dd}, J=3.2,1.9 \mathrm{~Hz}, \operatorname{ArH}), 6.21(1 \mathrm{H}, \mathrm{dd}, J=$ 3.2, $0.9 \mathrm{~Hz}, \operatorname{ArH}), 5.98$ (1H, br s, OH), $3.23\left(1 \mathrm{H}, \mathrm{q}, J=7.1 \mathrm{~Hz}, \mathrm{CH}_{3} \mathrm{CH}\right), 2.94\left(3 \mathrm{H}, \mathrm{s}, \mathrm{NCH}_{3}\right), 2.80$ $\left(3 \mathrm{H}, \mathrm{s}, \mathrm{NCH}_{3}\right), 1.47\left(3 \mathrm{H}, \mathrm{s}, \mathrm{CH}_{3} \mathrm{COH}\right), 1.28\left(3 \mathrm{H}, \mathrm{d}, J=7.1 \mathrm{~Hz}, \mathrm{CH}_{3} \mathrm{CH}\right) ;{ }^{13} \mathrm{C} \mathrm{NMR}(62.9 \mathrm{MHz}$, $\left.\mathrm{CDCl}_{3}\right) \delta 177.0(\mathrm{C}), 161.0(\mathrm{C}), 140.7(\mathrm{CH}), 110.4(\mathrm{CH}), 104.2(\mathrm{CH}), 72.4(\mathrm{C}), 41.0(\mathrm{CH}), 37.2\left(\mathrm{CH}_{3}\right)$, $35.2\left(\mathrm{CH}_{3}\right), 24.9\left(\mathrm{CH}_{3}\right), 11.5\left(\mathrm{CH}_{3}\right)$; HRMS (ES) Exact mass calcd for $\mathrm{C}_{11} \mathrm{H}_{18} \mathrm{NO}_{3}[\mathrm{M}+\mathrm{H}]^{+}$: 212.1281, found: 212.1281 .

\section{Reductive Aldol Reactions Using 4-Acryloylmorpholine: General Procedure B}

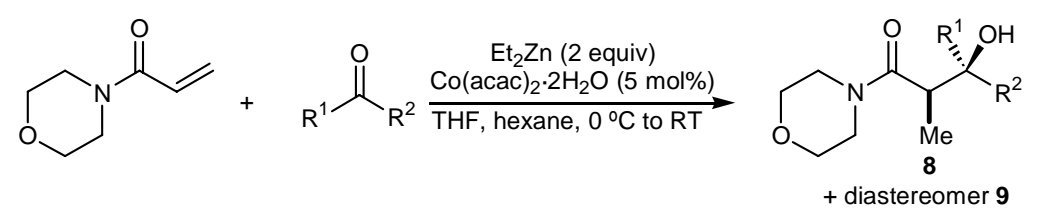

To a solution $\mathrm{Co}(\mathrm{acac})_{2} \cdot 2 \mathrm{H}_{2} \mathrm{O}(12.9 \mathrm{mg}, 0.05 \mathrm{mmol})$, 4-acryloylmorpholine $(126 \mu \mathrm{L}, 1.00 \mathrm{mmol})$ and the appropriate ketone $(1.10 \mathrm{mmol})$ in $\mathrm{THF}(10 \mathrm{~mL})$ at $0{ }^{\circ} \mathrm{C}$ was added $\mathrm{Et}_{2} \mathrm{Zn}(1 \mathrm{M}$ solution in hexane, $2.0 \mathrm{~mL}, 2.0 \mathrm{mmol}$ ) dropwise over $1 \mathrm{~min}$. The reaction was stirred at $0{ }^{\circ} \mathrm{C}$ until complete consumption of 4-acryloylmorpholine as observed by TLC analysis. The reaction was quenched carefully with saturated aqueous $\mathrm{NH}_{4} \mathrm{Cl}$ solution $(3 \mathrm{~mL})$ and the resulting mixture was stirred for 15 minutes. Further saturated aqueous $\mathrm{NH}_{4} \mathrm{Cl}$ solution $(30 \mathrm{~mL})$ was added, and the mixture was then extracted with $\mathrm{CH}_{2} \mathrm{Cl}_{2}$ $(3 \times 30 \mathrm{~mL})$. The combined organic layers were dried $\left(\mathrm{MgSO}_{4}\right)$ and concentrated in vacuo. Purification of the residue by column chromatography (EtOAc/petrol) afforded the aldol product(s).

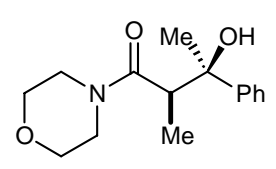

( \pm )-(2R,3R)-3-Hydroxy-2-methyl-1-morpholin-4-yl-3-phenylbutan-1-one

(8a).

The title compound was prepared according to General Procedure B from acetophenone $(127 \mu \mathrm{L}, 1.10 \mathrm{mmol})$ for a reaction time of $4 \mathrm{~h}$ and purified by column chromatography (20\% EtOAc/petrol) to give a white solid $(210 \mathrm{mg}, 80 \%)$ m.p. 129-131 ${ }^{\circ} \mathrm{C}$; IR $\left(\mathrm{CHCl}_{3}\right) 3366(\mathrm{OH}), 2971,2928,2856,1614(\mathrm{C}=\mathrm{O}), 1462,1445,1231,1117,1022 \mathrm{~cm}^{-1} ;{ }^{1} \mathrm{H}$ NMR $(360$ $\left.\mathrm{MHz}_{\mathrm{CDCl}}\right) \delta$ 7.48-7.45 (2H, m, ArH), 7.39-7.33 (2H, m, ArH), 7.27-7.22 (1H, m, ArH), 5.66 (1H, br s, OH), 3.81-3.54 (8H, m, $\left.2 \times \mathrm{OCH}_{2} \mathrm{CH}_{2} \mathrm{~N}\right), 2.96\left(1 \mathrm{H}, \mathrm{q}, J=7.1 \mathrm{~Hz}, \mathrm{CH}_{3} \mathrm{CH}\right), 1.55(3 \mathrm{H}, \mathrm{s}$, 
$\left.\mathrm{CH}_{3} \mathrm{COH}\right), 0.91\left(3 \mathrm{H}, \mathrm{d}, J=7.1 \mathrm{~Hz}, \mathrm{CH}_{3} \mathrm{CH}\right) ;{ }^{13} \mathrm{C} \mathrm{NMR}\left(62.9 \mathrm{MHz} \mathrm{CDCl}_{3}\right) \delta 176.0(\mathrm{C}), 145.7(\mathrm{C})$, $127.9(2 \times \mathrm{CH}), 126.4(\mathrm{CH}), 124.8(2 \times \mathrm{CH}), 74.6(\mathrm{C}), 66.8\left(\mathrm{CH}_{2}\right), 66.7\left(\mathrm{CH}_{2}\right), 46.2\left(\mathrm{CH}_{2}\right), 43.3(\mathrm{CH})$, $41.8\left(\mathrm{CH}_{2}\right), 29.8\left(\mathrm{CH}_{3}\right), 12.9\left(\mathrm{CH}_{3}\right)$; HRMS (ES) Exact mass calcd for $\mathrm{C}_{15} \mathrm{H}_{22} \mathrm{NO}_{3}[\mathrm{M}+\mathrm{H}]^{+}:$264.1594, found: 264.1590 .

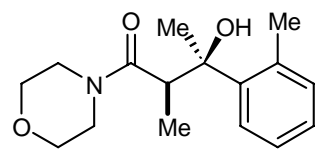

( \pm )-(2R,3R)-3-Hydroxy-2-methyl-3-(2-methylphenyl)-1-morpholin-4-ylbutan-

1-one (8b). The title compound was prepared according to General Procedure B from 2'-methylacetophenone $(144 \mu \mathrm{L}, 1.10 \mathrm{mmol})$ for a reaction time of $4 \mathrm{~h}$ and purified by column chromatography (20\% EtOAc/petrol) to give a colorless oil (231 mg, 84\%). IR (film) $3349(\mathrm{OH}), 2972,2928,2856,1614(\mathrm{C}=\mathrm{O}), 1464,1439,1230,1117,1020 \mathrm{~cm}^{-1}$; ${ }^{1} \mathrm{H}$ NMR (360 $\left.\mathrm{MHz}_{\mathrm{CDCl}}\right) \delta 7.68(1 \mathrm{H}, \mathrm{d}, J=\mathrm{Hz}, \mathrm{ArH}), 7.22-7.10(3 \mathrm{H}, \mathrm{m}, \operatorname{ArH}), 5.74(1 \mathrm{H}$, br s, OH), 3.81-3.55 $\left(8 \mathrm{H}, \mathrm{m}, 2 \times \mathrm{OCH}_{2} \mathrm{CH}_{2} \mathrm{~N}\right), 3.31\left(1 \mathrm{H}, \mathrm{q}, J=7.0 \mathrm{~Hz}, \mathrm{CH}_{3} \mathrm{CH}\right), 2.52\left(3 \mathrm{H}, \mathrm{s}, \mathrm{ArCH}_{3}\right), 1.63(3 \mathrm{H}, \mathrm{s}$, $\left.\mathrm{CH}_{3} \mathrm{COH}\right), 0.96\left(3 \mathrm{H}, \mathrm{d}, J=7.0 \mathrm{~Hz}, \mathrm{CH}_{3} \mathrm{CH}\right) ;{ }^{13} \mathrm{C} \mathrm{NMR}\left(62.9 \mathrm{MHz} \mathrm{CDCl}_{3}\right) \delta 176.0(\mathrm{C}), 143.1(\mathrm{C})$, $133.5(\mathrm{C}), 132.4(\mathrm{CH}), 126.9(\mathrm{CH}), 126.7(\mathrm{CH}), 125.7(\mathrm{CH}), 75.8(\mathrm{C}), 66.8\left(\mathrm{CH}_{2}\right), 66.7\left(\mathrm{CH}_{2}\right), 46.3$ $\left(\mathrm{CH}_{2}\right), 42.0(\mathrm{CH}), 40.8\left(\mathrm{CH}_{2}\right), 28.9\left(\mathrm{CH}_{3}\right), 22.8\left(\mathrm{CH}_{3}\right), 13.2\left(\mathrm{CH}_{3}\right)$; HRMS (ES) Exact mass calcd for $\mathrm{C}_{16} \mathrm{H}_{24} \mathrm{NO}_{3}[\mathrm{M}+\mathrm{H}]^{+}: 278.1751$, found: 278.1750 .

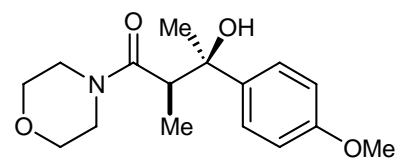

( \pm )-(2R,3R)-3-Hydroxy-3-(4-methoxyphenyl)-2-methyl-1-morpholin-4-

ylbutan-1-one (8c). The title compound was prepared according to General Procedure B from 4'-methoxyacetophenone (165 $\mathrm{mg}, 1.10 \mathrm{mmol})$ for a reaction time of $6 \mathrm{~h}$ and purified by column chromatography (20\% EtOAc/petrol) to give a white solid (249 mg, 85\%). m.p. 77-79 ${ }^{\circ} \mathrm{C}$; IR $\left(\mathrm{CHCl}_{3}\right) 3366$ (OH), 2970, 2931, 2856, 1612 (C=O), 1513, 1464, 1246, 1232, $1117 \mathrm{~cm}^{-1} ;{ }^{1} \mathrm{H}$ NMR $\left(360 \mathrm{MHz}, \mathrm{CDCl}_{3}\right) \delta 7.37(2 \mathrm{H}, \mathrm{dm}, J=9.0 \mathrm{~Hz}, \mathrm{ArH}), 6.89(2 \mathrm{H}, \mathrm{dm}, J$ $=9.0 \mathrm{~Hz}, \mathrm{ArH}), 5.58(1 \mathrm{H}$, br s, OH$), 3.82\left(3 \mathrm{H}, \mathrm{s}, \mathrm{OCH}_{3}\right) 3.79-3.53\left(8 \mathrm{H}, \mathrm{m}, 2\right.$ x $\left.\mathrm{OCH}_{2} \mathrm{CH}_{2} \mathrm{~N}\right), 2.90$ $\left(1 \mathrm{H}, \mathrm{q}, J=7.1 \mathrm{~Hz}, \mathrm{CH}_{3} \mathrm{CH}\right), 1.53\left(3 \mathrm{H}, \mathrm{s}, \mathrm{CH}_{3} \mathrm{COH}\right), 0.91\left(3 \mathrm{H}, \mathrm{d}, J=7.1 \mathrm{~Hz}, \mathrm{CH} \mathbf{H}_{3} \mathrm{CH}\right) ; 176.3(\mathrm{C})$, $158.1(\mathrm{C}), 138.0(\mathrm{C}), 126.0(2 \times \mathrm{CH}), 113.3(2 \times \mathrm{CH}), 74.4(\mathrm{C}), 66.8\left(\mathrm{CH}_{2}\right), 66.7\left(\mathrm{CH}_{2}\right), 55.1\left(\mathrm{CH}_{3}\right)$, $46.3\left(\mathrm{CH}_{2}\right), 43.5(\mathrm{CH}), 41.9\left(\mathrm{CH}_{2}\right), 29.9\left(\mathrm{CH}_{3}\right), 12.9\left(\mathrm{CH}_{3}\right)$; HRMS (ES) Exact mass calcd for $\mathrm{C}_{16} \mathrm{H}_{24} \mathrm{NO}_{4}[\mathrm{M}+\mathrm{H}]^{+}: 294.1700$, found: 294.1700 . 
$( \pm)-(2 R, 3 R)-3-H y d r o x y-2-m e t h y l-1-m o r p h o l i n-4-y l-3-n a p h t h a l e n-2-y l b u t a n-1-o n e ~(8 d)$ and ( \pm )(2R,3S)-3-hydroxy-2-methyl-1-morpholin-4-yl-3-naphthalen-2-ylbutan-1-one (9d).
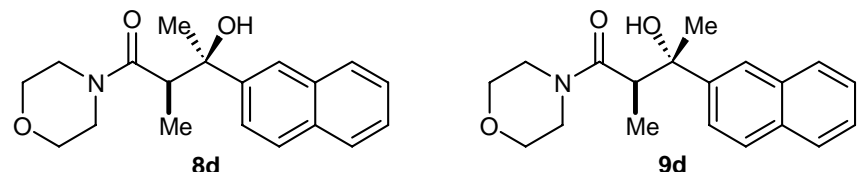

General Procedure B was followed using 2-acetonaphthone $(187 \mathrm{mg}, 1.10 \mathrm{mmol})$ for a reaction time of $4 \mathrm{~h}$ and the reaction mixture was purified by column chromatography (20\% EtOAc/petrol) to give the aldol product $\mathbf{8 d}(255 \mathrm{mg}, 82 \%)$ as a white solid followed by the aldol product $9 \mathbf{d}(54 \mathrm{mg}, 17 \%)$ as an off-white solid.

Data for 8d: m.p. 97-99 ${ }^{\circ} \mathrm{C}$; IR $\left(\mathrm{CHCl}_{3}\right) 3358(\mathrm{OH}), 2972,2927,2856,1612(\mathrm{C}=\mathrm{O}), 1468,1437,1231$, $1117,1026 \mathrm{~cm}^{-1} ;{ }^{1} \mathrm{H}$ NMR $\left(360 \mathrm{MHz}, \mathrm{CDCl}_{3}\right) \delta 8.04(1 \mathrm{H}, \mathrm{s}, \operatorname{ArH}), 7.90-7.82(3 \mathrm{H}, \mathrm{m}, \operatorname{ArH}), 7.52-7.44$ $(3 \mathrm{H}, \mathrm{m}, \mathrm{ArH}), 5.87(1 \mathrm{H}$, br s, OH $), 3.84-3.58\left(8 \mathrm{H}, \mathrm{m}, 2 \times \mathrm{OCH}_{2} \mathrm{CH}_{2} \mathrm{~N}\right), 3.09(1 \mathrm{H}, \mathrm{q}, J=7.1 \mathrm{~Hz}$, $\left.\mathrm{CH}_{3} \mathrm{CH}\right), 1.64\left(3 \mathrm{H}, \mathrm{s}, \mathrm{CH}_{3} \mathrm{COH}\right), 0.92\left(3 \mathrm{H}, \mathrm{d}, J=7.1 \mathrm{~Hz}, \mathrm{CH}_{3} \mathrm{CH}\right) ;{ }^{13} \mathrm{C} \mathrm{NMR}\left(62.9 \mathrm{MHz} \mathrm{CDCl}_{3}\right) \delta$ 176.0 (C), 143.0 (C), $133.1(\mathrm{C}), 132.1$ (C), $128.1(\mathrm{CH}), 127.7(\mathrm{CH}), 127.3(\mathrm{CH}), 125.9(\mathrm{CH}), 125.6$ $(\mathrm{CH}), 123.9(\mathrm{CH}), 123.0(\mathrm{CH}), 74.9(\mathrm{C}), 66.8\left(\mathrm{CH}_{2}\right), 66.7\left(\mathrm{CH}_{2}\right), 46.3\left(\mathrm{CH}_{2}\right), 43.1(\mathrm{CH}), 41.9\left(\mathrm{CH}_{2}\right)$, $29.9\left(\mathrm{CH}_{3}\right), 13.0\left(\mathrm{CH}_{3}\right)$; HRMS (ES) Exact mass calcd for $\mathrm{C}_{19} \mathrm{H}_{24} \mathrm{NO}_{3}[\mathrm{M}+\mathrm{H}]^{+}: 314.1751$, found: 314.1749 .

Data for 9d: m.p. 105-107 ${ }^{\circ} \mathrm{C}$; IR $\left(\mathrm{CHCl}_{3}\right) 3349(\mathrm{OH}), 2973,2924,2854,1609(\mathrm{C}=\mathrm{O}), 1442,1233$, 1115, 1067, $1030 \mathrm{~cm}^{-1} ;{ }^{1} \mathrm{H}$ NMR $\left(360 \mathrm{MHz} \mathrm{CDCl}_{3}\right) \delta 7.90(1 \mathrm{H}, \mathrm{s}, \operatorname{ArH}), 7.83-7.79(3 \mathrm{H}, \mathrm{m}, \operatorname{ArH})$, 7.50-7.43 (3H, m, ArH), $6.00\left(1 \mathrm{H}\right.$, br s, OH), 3.47-3.14 (7H, m, 2 x OCH $\left.\mathrm{CH}_{2} \mathrm{~N}\right), 2.96-2.86(2 \mathrm{H}, \mathrm{m}$, $\mathrm{CH}_{3} \mathrm{CH}$ and $\left.\mathrm{OCH}_{2} \mathrm{CH}_{2} \mathrm{~N}\right), 1.58\left(3 \mathrm{H}, \mathrm{s}, \mathrm{CH}_{3} \mathrm{COH}\right), 1.41\left(3 \mathrm{H}, \mathrm{d}, J=7.0 \mathrm{~Hz}, \mathrm{CH}_{3} \mathrm{CH}\right) ;{ }^{13} \mathrm{C}$ NMR $(62.9$ $\left.\mathrm{MHz} \mathrm{CDCl}_{3}\right) \delta 175.4(\mathrm{C}), 145.9(\mathrm{C}), 133.1(\mathrm{C}), 132.1(\mathrm{C}), 128.0(\mathrm{CH}), 127.7(\mathrm{CH}), 127.4(\mathrm{CH}), 126.1$ $(\mathrm{CH}), 125.7(\mathrm{CH}), 123.3(\mathrm{CH}), 123.0(\mathrm{CH}), 74.9(\mathrm{C}), 66.4\left(\mathrm{CH}_{2}\right), 66.2\left(\mathrm{CH}_{2}\right), 46.0\left(\mathrm{CH}_{2}\right), 42.6(\mathrm{CH})$, $41.5\left(\mathrm{CH}_{2}\right), 27.1\left(\mathrm{CH}_{3}\right), 12.6\left(\mathrm{CH}_{3}\right)$; HRMS (ES) Exact mass calcd for $\mathrm{C}_{19} \mathrm{H}_{24} \mathrm{NO}_{3}[\mathrm{M}+\mathrm{H}]^{+}:$314.1751, found: 314.1753 .

$( \pm)-(2 R, 3 R)-3-F u r a n-2-y l-3-h y d r o x y-2-m e t h y l-1-m o r p h o l i n-4-y l b u t a n-1-o n e ~(8 e)$ and $( \pm)-(2 R, 3 S)-$ 3-furan-2-yl-3-hydroxy-2-methyl-1-morpholin-4-ylbutan-1-one (9e).
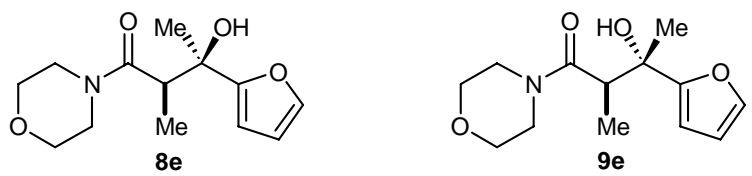

General Procedure B was followed using 2-furyl methyl ketone (121 mg, $1.10 \mathrm{mmol}$ ) for a reaction time of $4 \mathrm{~h}$ and the reaction mixture was purified by column chromatography (20\% EtOAc/petrol) to 
give the aldol product $\mathbf{8 e}(187 \mathrm{mg}, 72 \%)$ as a colorless oil followed by aldol product $9 \mathrm{e}(54 \mathrm{mg}, 22 \%)$ as a colorless oil.

Data for 8e: IR (film) $3388(\mathrm{OH}), 2976,2932,2858,1617(\mathrm{C}=\mathrm{O}), 1465,1440,1233,1117,1026 \mathrm{~cm}^{-1}$; ${ }^{1} \mathrm{H}$ NMR $\left(360 \mathrm{MHz}, \mathrm{CDCl}_{3}\right) \delta$ 7.35-7.34 (1H, m, CH), 6.38-6.37 (2H, m, 2 x CH), 5.40 (1H, br s, OH), 3.80-3.56 (8H, m, 2 x $\left.\mathrm{OCH}_{2} \mathrm{CH}_{2} \mathrm{~N}\right), 3.10\left(1 \mathrm{H}, \mathrm{q}, J=7.1 \mathrm{~Hz}, \mathrm{CH}_{3} \mathrm{CH}\right), 1.56\left(3 \mathrm{H}, \mathrm{s}, \mathrm{CH}_{3} \mathrm{COH}\right), 1.02$ $\left(3 \mathrm{H}, \mathrm{d}, J=7.1 \mathrm{~Hz}, \mathrm{CH}_{3} \mathrm{CH}\right) ;{ }^{13} \mathrm{C} \mathrm{NMR}\left(62.9 \mathrm{MHz}_{\mathrm{CDCl}}\right) \delta 175.5(\mathrm{C}), 157.6(\mathrm{C}), 140.8(\mathrm{CH}), 110.2$ $(\mathrm{CH}), 105.3(\mathrm{CH}), 73.4(\mathrm{C}), 66.8\left(\mathrm{CH}_{2}\right), 66.7\left(\mathrm{CH}_{2}\right), 46.2\left(\mathrm{CH}_{2}\right), 41.8\left(\mathrm{CH}_{2}\right), 41.2\left(\mathrm{CH}_{2}\right), 27.7\left(\mathrm{CH}_{3}\right)$, $13.1\left(\mathrm{CH}_{3}\right)$; HRMS (ES) Exact mass calcd for $\mathrm{C}_{13} \mathrm{H}_{20} \mathrm{NO}_{4}[\mathrm{M}+\mathrm{H}]^{+}:$254.1387, found: 254.1388.

Data for 9e: IR (film) $3409(\mathrm{OH}), 2979,2926,2860,1616(\mathrm{C}=\mathrm{O}), 1469,1445,1232,1115,1031 \mathrm{~cm}^{-1}$; ${ }^{1} \mathrm{H}$ NMR $\left(360 \mathrm{MHz}, \mathrm{CDCl}_{3}\right) \delta$ 7.29-7.28 (1H, m, ArH), 6.31-6.30 (1H, m, ArH), 6.24-6.23 (1H, m, $\operatorname{ArH}), 5.68\left(1 \mathrm{H}\right.$, br s, OH), 3.71-3.28 (8H, m, 2 x $\left.\mathrm{OCH}_{2} \mathrm{CH}_{2} \mathrm{~N}\right), 3.22\left(1 \mathrm{H}, \mathrm{q}, J=7.1 \mathrm{~Hz}, \mathrm{CH}_{3} \mathrm{CH}\right), 1.47$ $\left(3 \mathrm{H}, \mathrm{s}, \mathrm{CH}_{3} \mathrm{COH}\right), 1.29\left(3 \mathrm{H}, \mathrm{d}, J=7.1 \mathrm{~Hz}, \mathrm{CH}_{3} \mathrm{CH}\right) ;{ }^{13} \mathrm{C} \mathrm{NMR}\left(62.9 \mathrm{MHz}, \mathrm{CDCl}_{3}\right) \delta 175.6(\mathrm{C}), 160.9$ (C), $140.8(\mathrm{CH}), 110.5(\mathrm{CH}), 104.6(\mathrm{CH}), 72.5(\mathrm{C}), 66.7\left(\mathrm{CH}_{2}\right), 66.5\left(\mathrm{CH}_{2}\right), 46.2\left(\mathrm{CH}_{2}\right), 41.8\left(\mathrm{CH}_{2}\right)$, $40.5(\mathrm{CH}), 24.9\left(\mathrm{CH}_{3}\right), 11.8\left(\mathrm{CH}_{3}\right)$; HRMS (ES) Exact mass calcd for $\mathrm{C}_{12} \mathrm{H}_{24} \mathrm{NO}_{3}[\mathrm{M}+\mathrm{H}]^{+}: 230.1751$, found: 230.1749 .

$( \pm)-(2 R, 3 S)-3-H y d r o x y-2,3,4-t r i m e t h y l-1-m o r p h o l i n-4-y l p e n t a n-1-o n e \quad(8 f) \quad$ and $\quad( \pm)-(2 R, 3 R)-3-$ hydroxy-2,3,4-trimethyl-1-morpholin-4-ylpentan-1-one (9f).
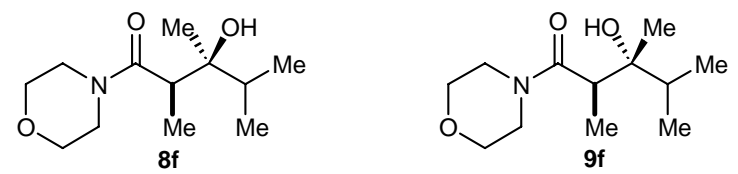

General Procedure B was followed using 3-methyl-2-butanone $(118 \mu \mathrm{L}, 1.10 \mathrm{mmol})$ for a reaction time of $4 \mathrm{~h}$ and the reaction mixture was purified by column chromatography ( $30 \%$ EtOAc/petrol) to give the aldol product $\mathbf{8 f}$ (75 $\mathrm{mg}, 33 \%)$ as a pale yellow solid followed by the aldol product $\mathbf{9 f}$ (71 $\mathrm{mg}$, $31 \%$ ) as a pale yellow oil. Slow evaporation of a hexane solution of $\mathbf{8 f}$ provided colorless crystalline crystals that were suitable for X-ray crystallography.

Data for 8f: m.p. $55-57^{\circ} \mathrm{C}$; IR $\left(\mathrm{CHCl}_{3}\right) 3398(\mathrm{OH}), 2964,1614(\mathrm{C}=\mathrm{O}), 1465,1371,1266,1231,1117$, 1031, $1011 \mathrm{~cm}^{-1} ;{ }^{1} \mathrm{H}$ NMR (360 MHz, $\left.\mathrm{CDCl}_{3}\right) \delta 5.31(1 \mathrm{H}, \mathrm{s}, \mathrm{OH}), 3.74-3.53\left(8 \mathrm{H}, \mathrm{m}, 2\right.$ x OCH $\left.\mathrm{CH}_{2} \mathrm{~N}\right)$, $2.78\left(1 \mathrm{H}, \mathrm{q}, J=7.1 \mathrm{~Hz}, \mathrm{CH}_{3} \mathrm{CHC}=\mathrm{O}\right), 2.01-1.94\left(1 \mathrm{H}, \mathrm{m}, \mathrm{CH}\left(\mathrm{CH}_{3}\right)_{2}\right), 1.21(3 \mathrm{H}, \mathrm{d}, J=7.1 \mathrm{~Hz}$, $\left.\mathrm{CH}_{3} \mathrm{CHC}=\mathrm{O}\right), 1.02\left(3 \mathrm{H}, \mathrm{s}, \mathrm{CH}_{3} \mathrm{COH}\right), 0.99\left(3 \mathrm{H}, \mathrm{d}, J=7.0 \mathrm{~Hz}, \mathrm{CH}\left(\mathrm{CH}_{3}\right)_{2}\right), 0.81(3 \mathrm{H}, \mathrm{d}, J=7.0 \mathrm{~Hz}$, $\left.\mathrm{CH}\left(\mathrm{CH}_{3}\right)_{2}\right) ;{ }^{13} \mathrm{C} \mathrm{NMR}\left(62.9 \mathrm{MHz}, \mathrm{CDCl}_{3}\right) \delta 176.7(\mathrm{C}), 74.4(\mathrm{C}), 66.9\left(\mathrm{CH}_{2}\right), 66.7\left(\mathrm{CH}_{2}\right), 46.3\left(\mathrm{CH}_{2}\right)$, $41.8\left(\mathrm{CH}_{2}\right), 38.9(\mathrm{CH}), 32.9(\mathrm{CH}), 20.0\left(\mathrm{CH}_{3}\right), 17.2\left(\mathrm{CH}_{3}\right), 16.3\left(\mathrm{CH}_{3}\right), 11.4\left(\mathrm{CH}_{3}\right)$; HRMS (ES) Exact mass calcd for $\mathrm{C}_{12} \mathrm{H}_{24} \mathrm{NO}_{3}[\mathrm{M}+\mathrm{H}]^{+}: 230.1751$, found: 230.1750 . 
Data for 9f: IR (film) $3399(\mathrm{OH}), 2963,1613(\mathrm{C}=\mathrm{O}), 1466,1301,1266,1231,1117,1030,919 \mathrm{~cm}^{-1}$; ${ }^{1} \mathrm{H}$ NMR $\left(360 \mathrm{MHz}, \mathrm{CDCl}_{3}\right) \delta 4.83(1 \mathrm{H}, \mathrm{s}, \mathrm{OH}), 3.75-3.49\left(8 \mathrm{H}, \mathrm{m}, 2 \times \mathrm{OCH}_{2} \mathrm{CH}_{2} \mathrm{~N}\right), 2.82(1 \mathrm{H}, \mathrm{q}, J=$ $\left.7.1 \mathrm{~Hz}, \mathrm{CH}_{3} \mathrm{CHC}=\mathrm{O}\right), 1.85-1.78\left(1 \mathrm{H}, \mathrm{m}, \mathrm{CH}\left(\mathrm{CH}_{3}\right)_{2}\right), 1.21\left(3 \mathrm{H}, \mathrm{d}, J=7.1 \mathrm{~Hz}, \mathrm{CH}_{3} \mathrm{CHC}=\mathrm{O}\right), 1.00(3 \mathrm{H}$, $\left.\mathrm{d}, J=5.4 \mathrm{~Hz}, \mathrm{CH}\left(\mathrm{CH}_{3}\right)_{2}\right) 0.98\left(3 \mathrm{H}, \mathrm{s}, \mathrm{CH}_{3} \mathrm{COH}\right), 0.80\left(3 \mathrm{H}, \mathrm{d}, J=6.9 \mathrm{~Hz}, \mathrm{CH}\left(\mathrm{CH}_{3}\right)_{2}\right) ;{ }^{13} \mathrm{C} \mathrm{NMR}(62.9$ $\left.\mathrm{MHz} \mathrm{CDCl}_{3}\right) \delta 176.4(\mathrm{C}), 75.5(\mathrm{C}), 66.8\left(\mathrm{CH}_{2}\right), 66.7\left(\mathrm{CH}_{2}\right), 46.1\left(\mathrm{CH}_{2}\right), 41.6\left(\mathrm{CH}_{2}\right), 38.7(\mathrm{CH}), 36.1$ $(\mathrm{CH}), 18.4\left(\mathrm{CH}_{3}\right), 17.3\left(\mathrm{CH}_{3}\right), 17.0\left(\mathrm{CH}_{3}\right), 12.7\left(\mathrm{CH}_{3}\right)$; HRMS (ES) Exact mass calcd for $\mathrm{C}_{12} \mathrm{H}_{24} \mathrm{NO}_{3}$ $[\mathrm{M}+\mathrm{H}]^{+}:$230.1751, found: 230.1749 .

$( \pm)-(2 R, 3 S)-3-H y d r o x y-2,3,5-t r i m e t h y l-1-m o r p h o l i n-4-y l h e x a n-1-o n e \quad(8 g) \quad$ and $\quad( \pm)-(2 R, 3 R)-3-$ hydroxy-2,3,5-trimethyl-1-morpholin-4-ylhexan-1-one (9g).
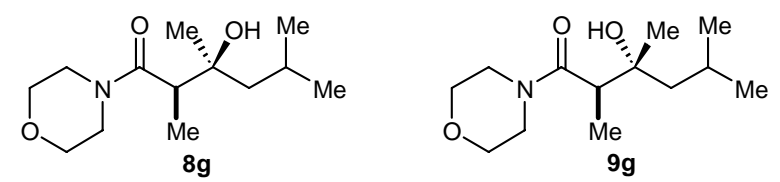

General Procedure B was followed using 4-methyl-2-pentanone (138 $\mu \mathrm{L}, 1.10 \mathrm{mmol})$ for a reaction time of $3 \mathrm{~h}$ and the reaction mixture was purified by column chromatography (10\% EtOAc/petrol) to give the aldol product $\mathbf{8 g}(86 \mathrm{mg}, 35 \%)$ as a colorless crystalline solid that was suitable for X-ray crystallography followed by the aldol product $9 \mathrm{~g}(87 \mathrm{mg}, 36 \%)$ as a colorless oil.

Data for 8g: m.p. $43-45^{\circ} \mathrm{C}$; IR (film) $3399(\mathrm{OH}), 2955,2867,1615(\mathrm{C}=\mathrm{O}), 1466,1439,1266,1228$, $1118,1026 \mathrm{~cm}^{-1} ;{ }^{1} \mathrm{H}$ NMR $\left(360 \mathrm{MHz}, \mathrm{CDCl}_{3}\right) \delta 4.49(1 \mathrm{H}$, br s, OH), 3.78-3.65 (6H, m, $2 \mathrm{x}$ $\left.\mathrm{OCH}_{2} \mathrm{CH}_{2} \mathrm{~N}\right), 3.63-3.50\left(2 \mathrm{H}, \mathrm{m}, 2 \times \mathrm{OCH}_{2} \mathrm{CH}_{2} \mathrm{~N}\right), 2.65\left(1 \mathrm{H}, \mathrm{q}, J=7.1 \mathrm{~Hz}, \mathrm{CH}_{3} \mathrm{CHC}=\mathrm{O}\right), 1.87-1.77$ $\left(1 \mathrm{H}, \mathrm{m}, \mathrm{CH}\left(\mathrm{CH}_{3}\right)_{2}\right), 1.53\left(1 \mathrm{H}, \mathrm{dd}, J=14.1,7.4 \mathrm{~Hz}, \mathrm{CH}_{2} \mathrm{CH}\left(\mathrm{CH}_{3}\right)_{2}\right), 1.33(1 \mathrm{H}, \mathrm{dd}, J=14.1,4.6 \mathrm{~Hz}$, $\left.\mathrm{CH}_{2} \mathrm{CH}\left(\mathrm{CH}_{3}\right)_{2}\right), 1.25\left(3 \mathrm{H}, \mathrm{s}, \mathrm{CH}_{3} \mathrm{COH}\right), 1.21\left(3 \mathrm{H}, \mathrm{d}, J=7.1 \mathrm{~Hz}, \mathrm{CH}_{3} \mathrm{CHC}=\mathrm{O}\right), 1.06(3 \mathrm{H}, \mathrm{d}, J=6.6$ $\left.\mathrm{Hz}, \mathrm{CH}\left(\mathrm{CH}_{3}\right)_{2}\right), 0.98\left(3 \mathrm{H}, \mathrm{d}, J=6.6 \mathrm{~Hz}, \mathrm{CH}\left(\mathrm{CH}_{3}\right)_{2}\right) ;{ }^{13} \mathrm{C} \mathrm{NMR}\left(62.9 \mathrm{MHz} \mathrm{CDCl}_{3}\right) \delta 176.4(\mathrm{C}), 72.9$ (C), $66.9\left(\mathrm{CH}_{2}\right), 66.7\left(\mathrm{CH}_{2}\right), 47.4\left(\mathrm{CH}_{2}\right), 46.3\left(\mathrm{CH}_{2}\right), 41.9(\mathrm{CH}), 41.7\left(\mathrm{CH}_{2}\right), 26.3\left(\mathrm{CH}_{3}\right), 25.1\left(\mathrm{CH}_{3}\right)$, $24.2\left(\mathrm{CH}_{3}\right), 23.9(\mathrm{CH}), 12.2\left(\mathrm{CH}_{3}\right)$; HRMS (ES) Exact mass calcd for $\mathrm{C}_{13} \mathrm{H}_{26} \mathrm{NO}_{3}[\mathrm{M}+\mathrm{H}]^{+}$: 244.1907, found: 244.1909 .

Data for 9g: IR (film) $2409(\mathrm{OH}), 2953,1615(\mathrm{C}=\mathrm{O}), 1457,1265,1229,1116,1028,848,750 \mathrm{~cm}^{-1} ;{ }^{1} \mathrm{H}$ NMR $\left(360 \mathrm{MHz}, \mathrm{CDCl}_{3}\right) \delta 4.36\left(1 \mathrm{H}\right.$, br s, OH), 3.71-3.43 $\left(8 \mathrm{H}, \mathrm{m}, 2\right.$ х $\left.\mathrm{OCH}_{2} \mathrm{CH}_{2} \mathrm{~N}\right), 2.57(1 \mathrm{H}, \mathrm{q}, J=$ $\left.7.1 \mathrm{~Hz}, \mathrm{CH}_{3} \mathrm{CHC}=\mathrm{O}\right), 2.60-2.54\left(1 \mathrm{H}, \mathrm{m}, \mathrm{CH}\left(\mathrm{CH}_{3}\right)_{2}\right), 1.38\left(1 \mathrm{H}, \mathrm{dd}, J=14.0,7.3 \mathrm{~Hz}, \mathrm{CH} \mathrm{CH}_{2}\left(\mathrm{CH}_{3}\right)_{2}\right)$, $1.27\left(1 \mathrm{H}, \mathrm{dd}, J=14.0,4.4 \mathrm{~Hz}, \mathrm{CH}_{2} \mathrm{CH}\left(\mathrm{CH}_{3}\right)_{2}\right), 1.16\left(3 \mathrm{H}, \mathrm{d}, J=7.1 \mathrm{~Hz}, \mathrm{CH}_{3} \mathrm{CHC}=\mathrm{O}\right), 1.13(3 \mathrm{H}, \mathrm{s}$, $\left.\mathrm{CH}_{3} \mathrm{COH}\right), 0.96\left(3 \mathrm{H}, \mathrm{d}, J=6.7 \mathrm{~Hz}, \mathrm{CH}\left(\mathrm{CH}_{3}\right)_{2}\right), 0.87\left(3 \mathrm{H}, \mathrm{d}, J=6.7 \mathrm{~Hz}, \mathrm{CH}\left(\mathrm{CH}_{3}\right)_{2}\right) ;{ }^{13} \mathrm{C} \mathrm{NMR}(62.9$ $\left.\mathrm{MHz} \mathrm{CDCl}_{3}\right) \delta 176.4(\mathrm{C}), 73.3(\mathrm{C}), 66.8\left(\mathrm{CH}_{2}\right), 66.6\left(\mathrm{CH}_{2}\right), 50.3\left(\mathrm{CH}_{2}\right), 46.1\left(\mathrm{CH}_{2}\right), 41.9(\mathrm{CH}), 41.6$ 
$\left(\mathrm{CH}_{2}\right), 25.2\left(\mathrm{CH}_{3}\right), 24.2\left(\mathrm{CH}_{3}\right.$ and $\left.\mathrm{CH}\right), 23.4\left(\mathrm{CH}_{3}\right), 12.4\left(\mathrm{CH}_{3}\right)$; HRMS (ES) Exact mass calcd for $\mathrm{C}_{13} \mathrm{H}_{26} \mathrm{NO}_{3}[\mathrm{M}+\mathrm{H}]^{+}: 244.1907$, found: 244.1909 .

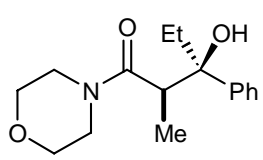

( \pm )-(2R,3R)-3-Hydroxy-2-methyl-1-morpholin-4-yl-3-phenylpentan-1-one

(8h).

The title compound was prepared according to General Procedure B from propiophenone ( $132 \mu \mathrm{L}, 1.10 \mathrm{mmol})$ for a reaction time of $7 \mathrm{~h}$ and purified by column chromatography (30\% EtOAc/petrol) to give a white solid (207 mg, 75\%). m.p. 147-149 ${ }^{\circ} \mathrm{C}$; IR $\left(\mathrm{CHCl}_{3}\right) 3349(\mathrm{OH}), 2970,2932,2857,1612(\mathrm{C}=\mathrm{O}), 1447,1230,1117,1028,703 \mathrm{~cm}^{-1} ;{ }^{1} \mathrm{H}$ NMR $(360$ $\left.\mathrm{MHz}, \mathrm{CDCl}_{3}\right) \delta$ 7.42-7.33 (4H, m, ArH), 7.26-7.21 (1H, m, ArH), $5.42(1 \mathrm{H}, \mathrm{s}, \mathrm{OH}), 3.81-3.56(8 \mathrm{H}, \mathrm{m}$, 2 x $\left.\mathrm{OCH}_{2} \mathrm{CH}_{2} \mathrm{~N}\right), 2.97\left(1 \mathrm{H}, \mathrm{q}, J=7.1 \mathrm{~Hz}, \mathrm{CH}_{3} \mathrm{CH}\right), 1.90-1.71\left(2 \mathrm{H}, \mathrm{m}, \mathrm{CH}_{3} \mathrm{CH}_{2} \mathrm{C}\right), 0.89(3 \mathrm{H}, \mathrm{d}, J=7.1$ $\left.\mathrm{Hz}, \mathrm{CH}_{3} \mathrm{CH}\right), 0.67\left(3 \mathrm{H}, \mathrm{t}, J=7.3 \mathrm{~Hz}, \mathrm{CH}_{3} \mathrm{CH}_{2} \mathrm{C}\right) ;{ }^{13} \mathrm{C}$ NMR $\left(62.9 \mathrm{MHz}, \mathrm{CDCl}_{3}\right) \delta 176.4(\mathrm{C}), 143.3$ (C), $128.0(2 \times \mathrm{CH}), 126.3(\mathrm{CH}), 125.6(2 \times \mathrm{CH}), 77.7(\mathrm{C}), 66.9\left(\mathrm{CH}_{2}\right), 66.8\left(\mathrm{CH}_{2}\right), 46.4\left(\mathrm{CH}_{2}\right), 43.2$ $(\mathrm{CH}), 41.9\left(\mathrm{CH}_{2}\right), 34.2\left(\mathrm{CH}_{2}\right), 13.0\left(\mathrm{CH}_{3}\right), 7.8\left(\mathrm{CH}_{3}\right)$; HRMS (ES) Exact mass calcd for $\mathrm{C}_{16} \mathrm{H}_{24} \mathrm{NO}_{3}$ $[\mathrm{M}+\mathrm{H}]^{+}: 278.1751$, found: 278.1754 .

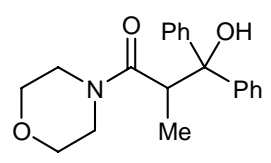

3-Hydroxy-2-methyl-1-morpholin-4-yl-3,3-diphenylpropan-1-one (8i). The title compound was prepared according to General Procedure B from benzophenone (200 $\mathrm{mg}, 1.10 \mathrm{mmol}$ ) for a reaction time of $3 \mathrm{~h}$ and purified by column chromatography (20\% EtOAc/petrol) to give a white solid (202 mg, 62\%). m.p. 108-110 ${ }^{\circ} \mathrm{C}$; IR $\left(\mathrm{CHCl}_{3}\right) 3324(\mathrm{OH})$, 2971, 2922, 2856, $1613(\mathrm{C}=\mathrm{O}), 1448,1230,1116,1032,751 \mathrm{~cm}^{-1} ;{ }^{1} \mathrm{H}$ NMR $\left(360 \mathrm{MHz}, \mathrm{CDCl}_{3}\right) \delta 7.52-$ 7.46 (4H, m, ArH), 7.33-7.26 (4H, m, ArH), 7.21-7.15 (2H, m, ArH), $6.43(1 \mathrm{H}, \mathrm{s}, \mathrm{OH}), 3.78(1 \mathrm{H}, \mathrm{q}, J$ $\left.=7.0 \mathrm{~Hz}, \mathrm{CH}_{3} \mathrm{CH}\right), 3.73-3.42\left(8 \mathrm{H}, \mathrm{m}, 2 \times \mathrm{OCH}_{2} \mathrm{CH}_{2} \mathrm{~N}\right), 1.17\left(1 \mathrm{H}, \mathrm{d}, J=7.0 \mathrm{~Hz}, \mathrm{CH}_{3} \mathrm{CH}\right) ;{ }^{13} \mathrm{C} \mathrm{NMR}$ $\left(62.9 \mathrm{MHz}, \mathrm{CDCl}_{3}\right) \delta 175.7(\mathrm{C}), 148.0(\mathrm{C}), 144.6(\mathrm{C}), 128.2(2 \times \mathrm{CH}), 128.1(2 \times \mathrm{CH}), 126.7(\mathrm{CH})$, $126.5(\mathrm{CH}), 125.5(2 \times \mathrm{CH}), 125.2(2 \times \mathrm{CH}), 78.6(\mathrm{C}), 66.7\left(\mathrm{CH}_{2}\right), 66.5\left(\mathrm{CH}_{2}\right), 46.1\left(\mathrm{CH}_{2}\right), 41.7\left(\mathrm{CH}_{2}\right)$, $41.4(\mathrm{CH}), 12.9\left(\mathrm{CH}_{3}\right)$; HRMS (ES) Exact mass calcd for $\mathrm{C}_{20} \mathrm{H}_{24} \mathrm{NO}_{3}[\mathrm{M}+\mathrm{H}]^{+}: 326.1751$, found: 326.1752 . 
Reductive Aldol Reactions Using $\beta$-Substituted $\alpha, \beta$-Unsaturated Morpholine Amides

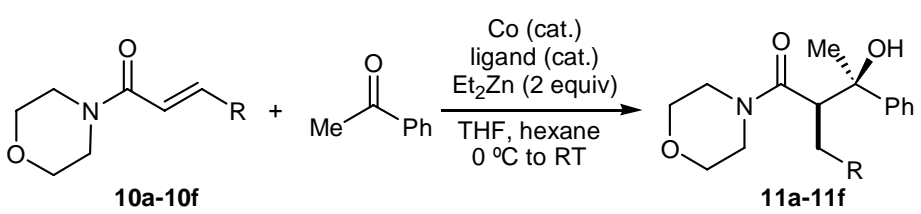

\section{Using Co(acac) $)_{2} \cdot 2 \mathrm{H}_{2} \mathrm{O} / \mathrm{Et}_{2} \mathrm{Zn}$ : General Procedure C}

To a solution $\mathrm{Co}(\mathrm{acac})_{2} \cdot 2 \mathrm{H}_{2} \mathrm{O}(12.9 \mathrm{mg}, 0.05 \mathrm{mmol})$, the appropriate $\alpha, \beta$-unsaturated morpholine amide $(1.00 \mathrm{mmol})$ and acetophenone $(127 \mu \mathrm{L}, 1.10 \mathrm{mmol})$ in THF $(10 \mathrm{~mL})$ at $0{ }^{\circ} \mathrm{C}$ was added $\mathrm{Et}_{2} \mathrm{Zn}$ (1 M solution in hexane, $2.0 \mathrm{~mL}, 2.0 \mathrm{mmol}$ ) in one portion. The reaction was stirred at $0{ }^{\circ} \mathrm{C}$ for $2 \mathrm{~min}$ and then an room temperature until complete consumption of the $\alpha, \beta$-unsaturated morpholine amide as observed by TLC analysis. The reaction was filtered through a short plug of $\mathrm{SiO}_{2}(\mathrm{ca} .4 \mathrm{~cm}$ high $\mathrm{x} 2 \mathrm{~cm}$ diameter) using EtOAc as eluent (ca. $50 \mathrm{~mL}$ ) and the filtrate was concentrated in vacuo. Purification of the residue by column chromatography (EtOAc/petrol) afforded the aldol product.

\section{Using $\mathrm{CoCl}_{2} / \mathrm{Cy}_{2} \mathrm{PPh} / \mathrm{Et}_{2} \mathrm{Zn}$ : General Procedure D}

To a solution $\mathrm{CoCl}_{2}(6.5 \mathrm{mg}, 0.05 \mathrm{mmol}), \mathrm{Cy}_{2} \mathrm{PPh}(15.9 \mathrm{mg}, 0.055 \mathrm{mmol})$, the appropriate $\alpha, \beta-$ unsaturated morpholine amide $(1.00 \mathrm{mmol})$ and acetophenone $(127 \mu \mathrm{L}, 1.10 \mathrm{mmol})$ in $\mathrm{THF}(10 \mathrm{~mL})$ at $0{ }^{\circ} \mathrm{C}$ was added $\mathrm{Et}_{2} \mathrm{Zn}(1 \mathrm{M}$ solution in hexane, $2.0 \mathrm{~mL}, 2.0 \mathrm{mmol})$ in one portion. The reaction was stirred at $0{ }^{\circ} \mathrm{C}$ for $2 \mathrm{~min}$ and then at room temperature until complete consumption of the $\alpha, \beta$ unsaturated morpholine amide as observed by TLC analysis. The reaction mixture was filtered through a short plug of $\mathrm{SiO}_{2}(\mathrm{ca} .4 \mathrm{~cm}$ high $\times 2 \mathrm{~cm}$ diameter) using EtOAc as eluent $(\mathrm{ca} .50 \mathrm{~mL})$ and the filtrate was concentrated in vacuo. Purification of the residue by column chromatography (EtOAc/petrol) afforded the aldol product.

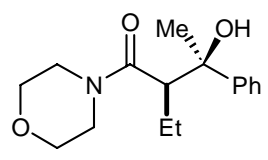

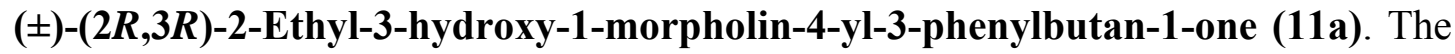
title compound was prepared according to General Procedure D from $\alpha, \beta$-unsaturated morpholine amide $\mathbf{1 0 a}(155 \mathrm{mg}, 1.00 \mathrm{mmol})$ for a reaction time of $2 \mathrm{~h}$ and purified by column chromatography (30\% EtOAc/petrol) to give a white solid $(210 \mathrm{mg}, 76 \%)$ m.p. $97-98{ }^{\circ} \mathrm{C}$; IR $\left(\mathrm{CHCl}_{3}\right) 3376(\mathrm{OH}), 2968,2858,1613(\mathrm{C}=\mathrm{O}), 1446,1230,1118,1034,849,703 \mathrm{~cm}^{-1} ;{ }^{1} \mathrm{H}$ NMR $(360$ $\left.\mathrm{MHz}_{\mathrm{CDCl}}\right) \delta$ 7.46-7.43 (2H, m, ArH), 7.33-7.29 (2H, m, ArH), 7.23-7.18 (1H, m, ArH), $5.33(1 \mathrm{H}$, br s, OH), 3.79-3.60 (8H, m, 2 x $\left.\mathrm{OCH}_{2} \mathrm{CH}_{2} \mathrm{~N}\right), 2.91\left(1 \mathrm{H}, \mathrm{dd}, J=10.9,3.6 \mathrm{~Hz}, \mathrm{CH}_{2} \mathrm{CH}\right), 1.83-1.69(1 \mathrm{H}$, m, $\left.\mathrm{CH}_{2} \mathrm{CH}_{3}\right), 1.46\left(3 \mathrm{H}, \mathrm{s}, \mathrm{CH}_{3} \mathrm{COH}\right), 1.22-1.11\left(1 \mathrm{H}, \mathrm{m}, \mathrm{CH}_{2} \mathrm{CH}_{3}\right), 0.68\left(3 \mathrm{H}, \mathrm{t}, J=7.5 \mathrm{~Hz}, \mathrm{CH}_{2} \mathrm{CH}_{3}\right)$; 
${ }^{13} \mathrm{C}$ NMR (62.9 MHz $\left.\mathrm{CDCl}_{3}\right) \delta 175.0(\mathrm{C}), 145.9(\mathrm{C}), 127.9(2 \times \mathrm{CH}), 126.4(\mathrm{CH}), 124.7(2 \times \mathrm{CH}), 74.6$ (C), $66.8\left(\mathrm{CH}_{2}\right), 66.5\left(\mathrm{CH}_{2}\right), 50.7(\mathrm{CH}), 46.6\left(\mathrm{CH}_{2}\right), 41.9\left(\mathrm{CH}_{2}\right), 29.8\left(\mathrm{CH}_{3}\right), 21.2\left(\mathrm{CH}_{2}\right), 12.0\left(\mathrm{CH}_{3}\right)$; HRMS (ES) Exact mass calcd for $\mathrm{C}_{16} \mathrm{H}_{24} \mathrm{NO}_{3}[\mathrm{M}+\mathrm{H}]^{+}: 278.1751$, found: 278.1751 .

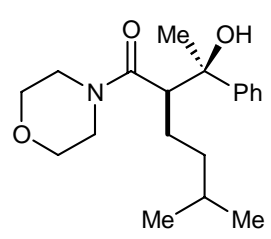

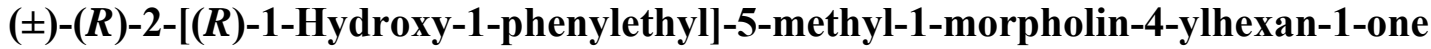
(11b). The title compound was prepared according to General Procedure D from $\alpha, \beta$ unsaturated morpholine amide $\mathbf{1 0 b}(197 \mathrm{mg}, 1.00 \mathrm{mmol})$ for a reaction time of $5 \mathrm{~h}$ and purified by column chromatography ( $30 \% \mathrm{EtOAc/petrol)}$ to give a white solid (270 mg, 85\%). m.p. $75-76{ }^{\circ} \mathrm{C}$; IR $\left(\mathrm{CHCl}_{3}\right) 3377(\mathrm{OH}), 2958,2866,1613(\mathrm{C}=\mathrm{O}), 1463,1232,1118$, $1035,849,703 \mathrm{~cm}^{-1} ;{ }^{1} \mathrm{H}$ NMR $\left(360 \mathrm{MHz}, \mathrm{CDCl}_{3}\right) \delta$ 7.45-7.43 (2H, m, ArH), 7.33-7.29 (2H, m, ArH), 7.23-7.18 $(1 \mathrm{H}, \mathrm{m}, \mathrm{ArH}), 5.26(1 \mathrm{H}, \mathrm{br} \mathrm{s}, \mathrm{OH}), 3.75-3.59\left(8 \mathrm{H}, \mathrm{m}, 2\right.$ х $\left.\mathrm{OCH}_{2} \mathrm{CH}_{2} \mathrm{~N}\right), 2.94(1 \mathrm{H}, \mathrm{dd}, J=$ 10.7, $3.4 \mathrm{~Hz}, \mathrm{CHC}=\mathrm{O}), 1.80-1.69\left(1 \mathrm{H}, \mathrm{m}, \mathrm{CH}_{2} \mathrm{CHC}=\mathrm{O}\right), 1.46\left(3 \mathrm{H}, \mathrm{s}, \mathrm{CH}_{3} \mathrm{COH}\right), 1.35-1.24(1 \mathrm{H}, \mathrm{m}$, $\left.\mathrm{CH}_{2} \mathrm{CHC}=\mathrm{O}\right), 1.19-1.10\left(1 \mathrm{H}, \mathrm{m}, \mathrm{CH}\left(\mathrm{CH}_{3}\right)_{2}\right), 1.00-0.89\left(1 \mathrm{H}, \mathrm{m}, \mathrm{CH}_{2} \mathrm{CH}\left(\mathrm{CH}_{3}\right)_{2}\right), 0.87-0.77(1 \mathrm{H}, \mathrm{m}$, $\left.\mathrm{CH}_{2} \mathrm{CH}\left(\mathrm{CH}_{3}\right)_{2}\right), 0.69\left(3 \mathrm{H}, \mathrm{t}, J=5.1 \mathrm{~Hz}, \mathrm{CH}\left(\mathrm{CH}_{3}\right)_{2}\right), 0.67\left(3 \mathrm{H}, \mathrm{t}, J=5.0 \mathrm{~Hz}, \mathrm{CH}\left(\mathrm{CH}_{3}\right)_{2}\right) ;{ }^{13} \mathrm{C} \mathrm{NMR}$ (62.9 $\left.\mathrm{MHz} \mathrm{CDCl}_{3}\right) \delta 175.2(\mathrm{C}), 145.9(\mathrm{C}), 127.9(2 \times \mathrm{CH}), 126.4(\mathrm{CH}), 124.7(2 \times \mathrm{CH}), 74.7(\mathrm{C}), 66.9$ $\left(\mathrm{CH}_{2}\right), 66.6\left(\mathrm{CH}_{2}\right), 49.2(\mathrm{CH}), 46.6\left(\mathrm{CH}_{2}\right), 42.0\left(\mathrm{CH}_{2}\right), 36.7\left(\mathrm{CH}_{3}\right), 29.9\left(\mathrm{CH}_{2}\right), 27.8(\mathrm{CH}), 26.0\left(\mathrm{CH}_{2}\right)$, $22.5\left(\mathrm{CH}_{2}\right), 21.9\left(\mathrm{CH}_{3}\right)$; HRMS (ES) Exact mass calcd for $\mathrm{C}_{19} \mathrm{H}_{30} \mathrm{NO}_{3}[\mathrm{M}+\mathrm{H}]^{+}$: 320.2220, found: 320.2222 .

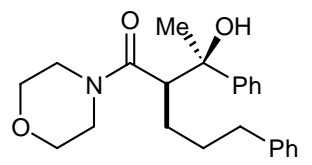

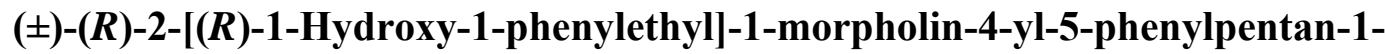
one (11c). The title compound was prepared according to General Procedure D from $\alpha, \beta$-unsaturated morpholine amide 10c $(245 \mathrm{mg}, 1.00 \mathrm{mmol})$ for a reaction time of $5 \mathrm{~h}$ and purified by column chromatography (30\% EtOAc/petrol) to give a 16:1 inseparable mixture of diastereoisomers as a white solid (298 mg, 81\%). m.p. $76-77{ }^{\circ} \mathrm{C}$; IR $\left(\mathrm{CHCl}_{3}\right) 3378(\mathrm{OH})$, 2969, 2857, $1609(\mathrm{C}=\mathrm{O}), 1445,1230,1116,1030,910,701 \mathrm{~cm}^{-1} ;{ }^{1} \mathrm{H} \mathrm{NMR}\left(360 \mathrm{MHz}, \mathrm{CDCl}_{3}\right) \delta 7.56-$ $7.54(2 \mathrm{H}, \mathrm{m}, \operatorname{ArH}), 7.43-7.39$ (2H, m, ArH), 7.35-7.17 (4H, m, ArH), 7.07-7.15 (2H, m, ArH), 5.34 $(1 \mathrm{H}, \mathrm{br} \mathrm{s}, \mathrm{OH}), 3.82-3.59\left(8 \mathrm{H}, \mathrm{m}, 2 \mathrm{x} \mathrm{OCH}_{2} \mathrm{CH}_{2} \mathrm{~N}\right), 3.07\left(1 \mathrm{H}, \mathrm{dd}, J=10.5,3.3 \mathrm{~Hz}, \mathrm{CH}_{2} \mathrm{CH}\right), 2.53-2.40$ $(2 \mathrm{H}, \mathrm{m}, \mathrm{CH} 2 \mathrm{Ar}), 1.99-1.87\left(1 \mathrm{H}, \mathrm{m}, \mathrm{CHCH}_{2}\right), 1.57\left(3 \mathrm{H}, \mathrm{s}, \mathrm{CH}_{3} \mathrm{COH}\right), 1.57-1.47\left(1 \mathrm{H}, \mathrm{m}, \mathrm{CHCH}_{2}\right)$, 1.39-1.25 (2H, m, $\left.\mathrm{CH}_{2} \mathrm{CH}_{2} \mathrm{CH}_{2}\right) ;{ }^{13} \mathrm{C}$ NMR (62.9 MHz CDCl $) \delta 174.8$ (C), 145.8 (C), 141.7 (C) 128.0 $(2 \times \mathrm{CH}), 127.9(4 \times \mathrm{CH}), 126.4(\mathrm{CH}), 125.5(\mathrm{CH}), 124.7(2 \times \mathrm{CH}), 74.6(\mathrm{C}), 66.7\left(\mathrm{CH}_{2}\right), 66.4\left(\mathrm{CH}_{2}\right)$, $49.0(\mathrm{CH}), 46.4\left(\mathrm{CH}_{2}\right), 41.8\left(\mathrm{CH}_{2}\right), 35.7\left(\mathrm{CH}_{2}\right), 29.6\left(\mathrm{CH}_{3}\right), 29.5\left(\mathrm{CH}_{2}\right), 27.9\left(\mathrm{CH}_{2}\right)$; HRMS (ES) Exact mass calcd for $\mathrm{C}_{23} \mathrm{H}_{30} \mathrm{NO}_{3}[\mathrm{M}+\mathrm{H}]^{+}: 368.2220$, found: 368.2223 . 


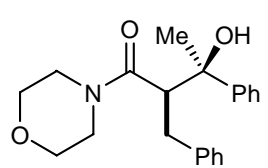

(2R,3R)-2-Benzyl-3-hydroxy-1-morpholin-4-yl-3-phenylbutan-1-one (11d). The

title compound was prepared according to General Procedure $\mathrm{C}$ from $\alpha, \beta$-unsaturated morpholine amide $10 \mathrm{~d}(217 \mathrm{mg}, 1.00 \mathrm{mmol})$ for a reaction time of $6 \mathrm{~h}$ and purified by column chromatography (30\% EtOAc/petrol) to give a white solid (241 mg, 71\%). m.p. 126-127 ${ }^{\circ} \mathrm{C}$; IR $\left(\mathrm{CHCl}_{3}\right) 3349(\mathrm{OH}), 2970,2859,1611(\mathrm{C}=\mathrm{O}), 1446,1233,1116,846,750,702 \mathrm{~cm}^{-1} ;{ }^{1} \mathrm{H}$ NMR $(360$ $\left.\mathrm{MHz}, \mathrm{CDCl}_{3}\right) \delta$ 7.58-7.56 (2H, m, ArH), 7.45-7.40 (2H, m, ArH), 7.33-7.28 (1H, m, ArH), 7.23-7.13 $(3 \mathrm{H}, \mathrm{m}, \mathrm{ArH}), 6.98-6.96(2 \mathrm{H}, \mathrm{m}, \mathrm{ArH}), 5.72(1 \mathrm{H}$, br s, OH $), 3.85-3.80\left(1 \mathrm{H}, \mathrm{m}, \mathrm{OCH}_{2} \mathrm{CH}_{2} \mathrm{~N}\right), 3.62-3.57$ $\left(1 \mathrm{H}, \mathrm{m}, \mathrm{OCH}_{2} \mathrm{CH}_{2} \mathrm{~N}\right), 3.37-3.14\left(5 \mathrm{H}, \mathrm{m}, 2\right.$ x $\left.\mathrm{OCH}_{2} \mathrm{CH}_{2} \mathrm{~N}\right), 2.99\left(1 \mathrm{H}\right.$, app t, $\left.J=12.5 \mathrm{~Hz}, \mathrm{CH}_{2} \mathrm{CH}\right)$, 2.89-2.83 (1H, m, $\left.\mathrm{OCH}_{2} \mathrm{CH}_{2} \mathrm{~N}\right), 2.51-2.40\left(2 \mathrm{H}, \mathrm{m}, \mathrm{CH}_{2} \mathrm{Ph}\right), 1.49\left(3 \mathrm{H}, \mathrm{s}, \mathrm{CH}_{3} \mathrm{COH}\right) ;{ }^{13} \mathrm{C} \mathrm{NMR}(62.9$

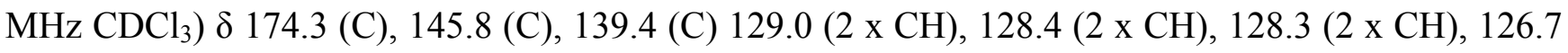
$(\mathrm{CH}), 126.5(\mathrm{CH}), 124.8(2 \times \mathrm{CH}), 74.8(\mathrm{C}), 66.4\left(\mathrm{CH}_{2}\right), 65.9\left(\mathrm{CH}_{2}\right), 52.1(\mathrm{CH}), 46.2\left(\mathrm{CH}_{2}\right), 41.8$ $\left(\mathrm{CH}_{2}\right)$, $34.7\left(\mathrm{CH}_{2}\right), 30.0\left(\mathrm{CH}_{3}\right)$; HRMS (ES) Exact mass calcd for $\mathrm{C}_{21} \mathrm{H}_{26} \mathrm{NO}_{3}[\mathrm{M}+\mathrm{H}]^{+}:$340.1907, found: 340.1909 .

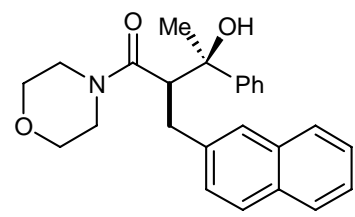

$( \pm)-(2 R, 3 R)-3-H y d r o x y-1-m o r p h o l i n-4-y l-2-n a p h t h a l e n-2-y l m e t h y l-3-$ phenylbutan-1-one (11e). The title compound was prepared according to

General Procedure $C$ from $\alpha, \beta$-unsaturated morpholine amide 10e (267 mg, 1.00 $\mathrm{mmol})$ for a reaction time of $6 \mathrm{~h}$ and purified by column chromatography (30\% EtOAc/petrol) to give a white solid (289 mg, 74\%). m.p. 137-139 ${ }^{\circ} \mathrm{C}$; IR $\left(\mathrm{CHCl}_{3}\right) 3357(\mathrm{OH}), 2970,2858,1610(\mathrm{C}=\mathrm{O}), 1446$, 1233, 1116, 909, 732, $703 \mathrm{~cm}^{-1} ;{ }^{1} \mathrm{H}$ NMR $\left(360 \mathrm{MHz}, \mathrm{CDCl}_{3}\right) \delta$ 7.78-7.76 (1H, m, ArH), 7.71-7.69 (2H, m, ArH), 7.65-7.63 (2H, m, ArH), 7.50-7.40 (5H, m, ArH), 7.37-7.33 (1H, m, ArH), 7.07 (1H, dd, $J=$ 8.4, $1.4 \mathrm{~Hz}, \operatorname{ArH}), 5.80\left(1 \mathrm{H}\right.$, br s, OH), 3.78-3.72 (1H, m, OCH $\left.\mathrm{CH}_{2} \mathrm{~N}\right), 3.53-3.47(1 \mathrm{H}, \mathrm{m}$, $\left.\mathrm{OCH}_{2} \mathrm{CH}_{2} \mathrm{~N}\right), 3.38-3.29\left(2 \mathrm{H}, \mathrm{m}, \mathrm{OCH}_{2} \mathrm{CH}_{2} \mathrm{~N}\right), 3.23-3.07\left(4 \mathrm{H}, \mathrm{m}, 2\right.$ x $\left.\mathrm{OCH}_{2} \mathrm{CH}_{2} \mathrm{~N}\right), 2.78-2.72(1 \mathrm{H}, \mathrm{m}$, $\left.\mathrm{CH}_{2} \mathrm{CH}\right), 2.61\left(1 \mathrm{H}, \mathrm{dd}, J=12.8,2.7 \mathrm{~Hz}, \mathrm{CHCH}_{2}\right), 2.21-2.15\left(1 \mathrm{H}, \mathrm{m}, \mathrm{CH}_{2} \mathrm{CH}\right), 1.54(3 \mathrm{H}, \mathrm{s}, \mathrm{CH} 3 \mathrm{COH})$; ${ }^{13} \mathrm{C}$ NMR (62.9 MHz $\left.\mathrm{CDCl}_{3}\right) \delta 174.2(\mathrm{C}), 145.8(\mathrm{C}), 136.7(\mathrm{C}), 133.3(\mathrm{C}), 131.9(\mathrm{C}), 128.2(2 \times \mathrm{CH})$, $127.9(\mathrm{CH}), 127.5(2 \times \mathrm{CH}), 127.1(2 \times \mathrm{CH}), 126.7(\mathrm{CH}), 126.2(\mathrm{CH}), 125.5(\mathrm{CH}), 124.8(\mathrm{CH}), 74.9$ (C), $66.3\left(\mathrm{CH}_{2}\right), 65.6\left(\mathrm{CH}_{2}\right), 52.0(\mathrm{CH}), 46.0\left(\mathrm{CH}_{2}\right), 41.7\left(\mathrm{CH}_{2}\right), 34.8\left(\mathrm{CH}_{2}\right), 29.9\left(\mathrm{CH}_{3}\right)$; HRMS (ES) Exact mass calcd for $\mathrm{C}_{25} \mathrm{H}_{28} \mathrm{NO}_{3}[\mathrm{M}+\mathrm{H}]^{+}: 390.2064$, found: 390.2070 .

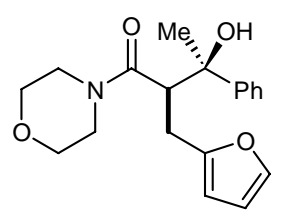

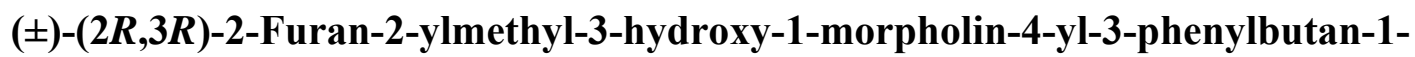
one (11f). The title compound was prepared according to General Procedure $\mathrm{C}$ from $\alpha, \beta$-unsaturated morpholine amide $\mathbf{1 0 f}(207 \mathrm{mg}, 1.00 \mathrm{mmol})$ for a reaction time of 6 
$\mathrm{h}$ and purified by column chromatography (30\% EtOAc/petrol) to give a white solid (278 $\mathrm{mg}, 84 \%)$. m.p. 65-66 ${ }^{\circ} \mathrm{C}$; IR $\left(\mathrm{CHCl}_{3}\right) 3366(\mathrm{OH}), 2970,2859,1613(\mathrm{C}=\mathrm{O}), 1446,1231,1116,919,735,703 \mathrm{~cm}^{-}$ 1. ${ }^{1} \mathrm{H}$ NMR (360 MHz, $\left.\mathrm{CDCl}_{3}\right) \delta$ 7.58-7.56 (2H, m, ArH), 7.43-7.39 (2H, m, ArH), 7.33-7.27 (1H, m, ArH), $7.26(1 \mathrm{H}, \mathrm{d}, J=1.5 \mathrm{~Hz}, \mathrm{CH}), 6.24(1 \mathrm{H}, \mathrm{dd}, J=3.1,1.5 \mathrm{~Hz}, \mathrm{CH}), 5.93(1 \mathrm{H}, \mathrm{d}, J=3.1 \mathrm{~Hz}, \mathrm{CH})$, $5.66\left(1 \mathrm{H}\right.$, br s, OH), 3.83-3.77 $\left(1 \mathrm{H}, \mathrm{m}, \mathrm{OCH}_{2} \mathrm{CH}_{2} \mathrm{~N}\right), 3.71-3.65\left(1 \mathrm{H}, \mathrm{m}, \mathrm{OCH}_{2} \mathrm{CH}_{2} \mathrm{~N}\right), 3.53-3.40(5 \mathrm{H}$, m, $\left.2 \times \mathrm{OCH}_{2} \mathrm{CH}_{2} \mathrm{~N}\right), 3.28-3.16\left(2 \mathrm{H}, \mathrm{m}, \mathrm{OCH}_{2} \mathrm{CH}_{2} \mathrm{~N}\right.$ and $\left.\mathrm{CHCH}_{2}\right), 3.11(1 \mathrm{H}, \mathrm{dd}, J=14.7,11.8 \mathrm{~Hz}$, $\left.\mathrm{CH}_{2} \mathrm{CH}\right), 2.45\left(1 \mathrm{H}, \mathrm{dd}, J=14.7,3.2 \mathrm{~Hz}, \mathrm{CH} \mathbf{H}_{2} \mathrm{CH}\right), 1.54\left(3 \mathrm{H}, \mathrm{s}, \mathrm{CH}_{3} \mathrm{COH}\right) ;{ }^{13} \mathrm{C} \mathrm{NMR}(62.9 \mathrm{MHz}$ $\left.\mathrm{CDCl}_{3}\right) \delta 174.2(\mathrm{C}), 153.1(\mathrm{C}), 145.3(\mathrm{C}), 141.1(\mathrm{CH}), 128.2(2 \times \mathrm{CH}), 126.7(\mathrm{CH}), 124.8(2 \times \mathrm{CH})$, $110.5(\mathrm{CH}), 106.7(\mathrm{CH}), 74.5(\mathrm{C}), 66.6\left(\mathrm{CH}_{2}\right), 66.3\left(\mathrm{CH}_{2}\right), 48.5(\mathrm{CH}), 46.2\left(\mathrm{CH}_{2}\right), 41.9\left(\mathrm{CH}_{2}\right), 30.0$ $\left(\mathrm{CH}_{3}\right), 27.0\left(\mathrm{CH}_{2}\right)$; HRMS (ES) Exact mass calcd for $\mathrm{C}_{19} \mathrm{H}_{24} \mathrm{NO}_{4}[\mathrm{M}+\mathrm{H}]^{+}:$330.1700, found: 330.1698 .

\section{Reductive Aldol Reactions Using $\alpha, \beta$-Unsaturated Morpholine Amide 12: General Procedure E}

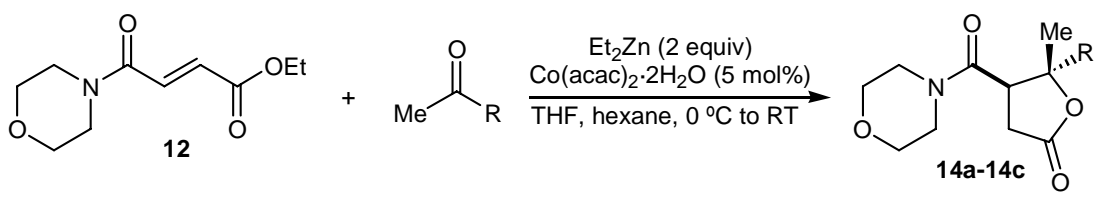

To a solution $\mathrm{Co}(\mathrm{acac})_{2} \cdot 2 \mathrm{H}_{2} \mathrm{O}(12.9 \mathrm{mg}, 0.05 \mathrm{mmol}), \alpha, \beta$-unsaturated morpholine amide $12(213 \mathrm{mg}$, $1.00 \mathrm{mmol})$ and the appropriate ketone $(1.10 \mathrm{mmol})$ in $\mathrm{THF}(10 \mathrm{~mL})$ at $0{ }^{\circ} \mathrm{C}$ was added $\mathrm{Et}_{2} \mathrm{Zn}(1 \mathrm{M}$ solution in hexane, $2.0 \mathrm{~mL}, 2.0 \mathrm{mmol}$ ) in one portion. The reaction was stirred at $0{ }^{\circ} \mathrm{C}$ for $2 \mathrm{~min}$ and then at room temperature until complete consumption of the $\alpha, \beta$-unsaturated morpholine amide 12 as observed by TLC analysis. The reaction mixture was filtered through a short plug of $\mathrm{SiO}_{2}(\mathrm{ca} .4 \mathrm{~cm}$ high $\times 2 \mathrm{~cm}$ diameter) using EtOAc as eluent (ca. $50 \mathrm{~mL})$ and the filtrate was concentrated in vacuo. Purification of the residue by column chromatography (EtOAc/petrol) afforded the product.

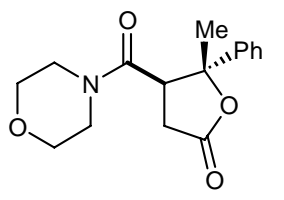

( \pm )-(4R,5R)-5-Methyl-4-(morpholine-4-carbonyl)-5-phenyltetrahydrofuran-2one (14a). The title compound was prepared according to General Procedure E from acetophenone $(127 \mu \mathrm{L}, 1.10 \mathrm{mmol})$ for a reaction time of $6 \mathrm{~h}$ and purified by column chromatography (80\% EtOAc/petrol) to give a white solid (196 mg, 68\%). m.p. 145-147 ${ }^{\circ} \mathrm{C}$; IR $\left(\mathrm{CHCl}_{3}\right) 3531(\mathrm{OH}), 2984,2859,1788(\mathrm{C}=\mathrm{O}), 1643(\mathrm{C}=\mathrm{O}), 1445,1244,1116,910,702 \mathrm{~cm}^{-1}$; ${ }^{1} \mathrm{H}$ NMR $\left(360 \mathrm{MHz} \mathrm{CDCl}_{3}\right) \delta$ 7.39-7.31 (5H, m, ArH), 3.76-3.39 (6H, m, 2 х $\left.\mathrm{OCH}_{2} \mathrm{CH}_{2} \mathrm{~N}\right), 3.32-3.19(2 \mathrm{H}, \mathrm{m}$, $\mathrm{CHCH}_{2}$ and $\left.\mathrm{CH}_{2} \mathrm{CH}\right), 3.14-3.07\left(1 \mathrm{H}, \mathrm{m}, \mathrm{OCH}_{2} \mathrm{CH}_{2} \mathrm{~N}\right), 2.92-2.86\left(1 \mathrm{H}, \mathrm{m}, \mathrm{OCH}_{2} \mathrm{CH}_{2} \mathrm{~N}\right), 2.59(1 \mathrm{H}, \mathrm{dd}, J$ $\left.=17.5,8.0 \mathrm{~Hz}, \mathrm{C} \mathbf{H}_{2} \mathrm{CH}\right), 1.67\left(3 \mathrm{H}, \mathrm{s}, \mathrm{CH}_{3} \mathrm{COH}\right) ;{ }^{13} \mathrm{C} \mathrm{NMR}\left(62.9 \mathrm{MHz} \mathrm{CDCl}_{3}\right) \delta 174.7(\mathrm{C}), 167.5(\mathrm{C})$, $143.1(\mathrm{C}), 128.9(2 \times \mathrm{CH}), 128.4(\mathrm{CH}), 124.4(2 \times \mathrm{CH}), 86.5(\mathrm{C}), 66.6\left(\mathrm{CH}_{2}\right), 66.2\left(\mathrm{CH}_{2}\right), 48.4(\mathrm{CH})$, 
$46.1\left(\mathrm{CH}_{2}\right), 42.4\left(\mathrm{CH}_{2}\right), 33.4\left(\mathrm{CH}_{2}\right), 22.5\left(\mathrm{CH}_{3}\right)$; HRMS (ES) Exact mass calcd for $\mathrm{C}_{16} \mathrm{H}_{23} \mathrm{~N}_{2} \mathrm{O}_{4}$ $\left[\mathrm{M}+\mathrm{NH}_{4}\right]^{+}: 307.1652$, found: 307.1648 .

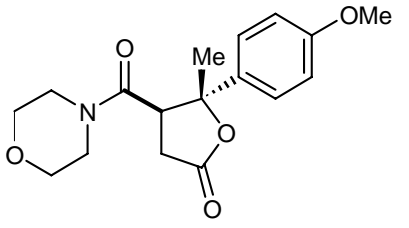

( \pm )-(4R,5R)-5-(4-Methoxyphenyl)-5-methyl-4-(morpholine-4carbonyl)tetrahydrofuran-2-one (14b). The title compound was prepared according to General Procedure E from 4'-methoxyacetophenone (165 mg, $1.10 \mathrm{mmol}$ ) for a reaction time of $6 \mathrm{~h}$ and purified by column chromatography (80\% EtOAc/petrol) to give a white solid (197 mg, 62\%). m.p. 136-138 ${ }^{\circ} \mathrm{C}$; IR $\left(\mathrm{CHCl}_{3}\right) 3524(\mathrm{OH}), 2967,2858,1785(\mathrm{C}=\mathrm{O}), 1645(\mathrm{C}=\mathrm{O}), 1460,1251,1117,834,733 \mathrm{~cm}^{-1} ;{ }^{1} \mathrm{H}$ NMR $\left(360 \mathrm{MHz}_{\mathrm{CDCl}}\right) \delta 7.32(2 \mathrm{H}, \mathrm{d}, J=8.9 \mathrm{~Hz}, \mathrm{ArH}), 6.92(2 \mathrm{H}, \mathrm{d}, J=8.9 \mathrm{~Hz}, \mathrm{ArH}), 3.80\left(3 \mathrm{H}, \mathrm{s}, \mathrm{OCH}_{3}\right)$, 3.78-3.41 (6H, m, $\left.2 \times \mathrm{OCH}_{2} \mathrm{CH}_{2} \mathrm{~N}\right), 3.33-3.25\left(2 \mathrm{H}, \mathrm{m}, \mathrm{CHCH}_{2}\right.$ and $\left.\mathrm{CH}_{2} \mathrm{CH}\right), 3.14-3.07(1 \mathrm{H}, \mathrm{m}$, $\left.\mathrm{OCH}_{2} \mathrm{CH}_{2} \mathrm{~N}\right), 2.93-2.86\left(1 \mathrm{H}, \mathrm{m}, \mathrm{OCH}_{2} \mathrm{CH}_{2} \mathrm{~N}\right), 2.63\left(1 \mathrm{H}, \mathrm{dd}, J=17.5,8.1 \mathrm{~Hz}, \mathrm{CH}{ }_{2} \mathrm{CH}\right), 1.66(3 \mathrm{H}, \mathrm{s}$, $\left.\mathrm{CH}_{3} \mathrm{COH}\right) ;{ }^{13} \mathrm{C}$ NMR $\left(62.9 \mathrm{MHz} \mathrm{CDCl}_{3}\right) \delta 174.7$ (C), $167.4(\mathrm{C}), 159.4(\mathrm{C}), 134(\mathrm{C}), 125.9(2 \times \mathrm{CH})$, $114.0(2 \times \mathrm{CH}), 86.3(\mathrm{C}), 66.5\left(\mathrm{CH}_{2}\right), 66.2\left(\mathrm{CH}_{2}\right), 55.2\left(\mathrm{CH}_{3}\right), 48.7(\mathrm{CH}), 46.1\left(\mathrm{CH}_{2}\right), 42.4\left(\mathrm{CH}_{2}\right), 33.4$ $\left(\mathrm{CH}_{2}\right), 22.3\left(\mathrm{CH}_{3}\right)$; HRMS (ES) Exact mass calcd for $\mathrm{C}_{17} \mathrm{H}_{25} \mathrm{~N}_{2} \mathrm{O}_{5}\left[\mathrm{M}+\mathrm{NH}_{4}\right]^{+}$: 337.1758 , found: 337.1758 .

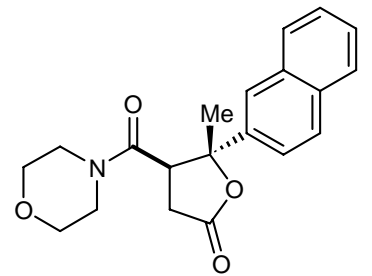

\section{$( \pm)-(4 R, 5 R)-5-M e t h y l-4-(m o r p h o l i n e-4-c a r b o n y l)-5-n a p h t h a l e n-2-$} yltetrahydrofuran-2-one (14c). The title compound was prepared according to General Procedure E from 2-acetonaphthone (165 $\mathrm{mg}, 1.10 \mathrm{mmol})$ for a reaction time of $6 \mathrm{~h}$ and purified by column chromatography $(80 \%$ EtOAc/petrol) to give a white solid (220 mg, 65\%). m.p. 144-146 ${ }^{\circ} \mathrm{C}$; IR $\left(\mathrm{CHCl}_{3}\right) 3526(\mathrm{OH}), 2984$, 2858, $1785(\mathrm{C}=\mathrm{O}), 1638(\mathrm{C}=\mathrm{O}), 1441,1238,1117,822,732 \mathrm{~cm}^{-1} ;{ }^{1} \mathrm{H}$ NMR $\left(360 \mathrm{MHz}, \mathrm{CDCl}_{3}\right) \delta 7.91-$ 7.82 (4H, m, ArH), 7.54-7.45 (3H, m, ArH), 3.82-3.49 (5H, m, 2 x OCH $\left.\mathrm{OH}_{2} \mathrm{~N}\right), 3.34-3.27$ (2H, m, $\mathrm{CHCH}_{2}$ and $\left.\mathrm{CH}_{2} \mathrm{CH}\right), 3.24-3.18\left(1 \mathrm{H}, \mathrm{m}, \mathrm{OCH}_{2} \mathrm{CH}_{2} \mathrm{~N}\right), 3.13-3.07\left(1 \mathrm{H}, \mathrm{m}, \mathrm{OCH}_{2} \mathrm{CH}_{2} \mathrm{~N}\right), 2.91-2.85(1 \mathrm{H}$, m, $\left.\mathrm{OCH}_{2} \mathrm{CH}_{2} \mathrm{~N}\right), 2.67\left(1 \mathrm{H}, \mathrm{dd}, J=17.5,8.1 \mathrm{~Hz}, \mathrm{CH}_{2} \mathrm{CH}\right), 1.80\left(3 \mathrm{H}, \mathrm{s}, \mathrm{CH}_{3} \mathrm{COH}\right) ;{ }^{13} \mathrm{C}$ NMR $(62.9$

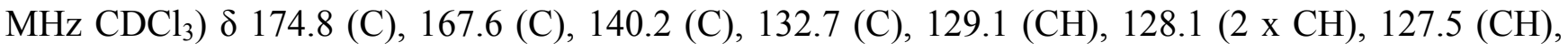
$126.9(\mathrm{CH}), 126.8(\mathrm{C}), 123.6(\mathrm{CH}), 122.0(\mathrm{CH}), 86.6(\mathrm{C}), 66.6\left(\mathrm{CH}_{2}\right), 66.2\left(\mathrm{CH}_{2}\right), 48.0(\mathrm{CH}), 46.2$ $\left(\mathrm{CH}_{2}\right), 42.5\left(\mathrm{CH}_{2}\right), 33.4\left(\mathrm{CH}_{2}\right), 22.7\left(\mathrm{CH}_{3}\right)$; HRMS (ES) Exact mass calcd for $\mathrm{C}_{20} \mathrm{H}_{25} \mathrm{~N}_{2} \mathrm{O}_{4}\left[\mathrm{M}+\mathrm{NH}_{4}\right]^{+}$: 357.1809, found: 357.1806 . 


\section{Stereochemical Determinations}

- The relative stereochemistry of the known aldol product 5a was assigned by comparison with literature spectral data. ${ }^{7}$

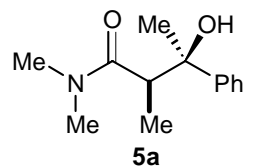

- The relative stereochemistry of $\mathbf{5 d}$ was determined by X-ray crystallography.
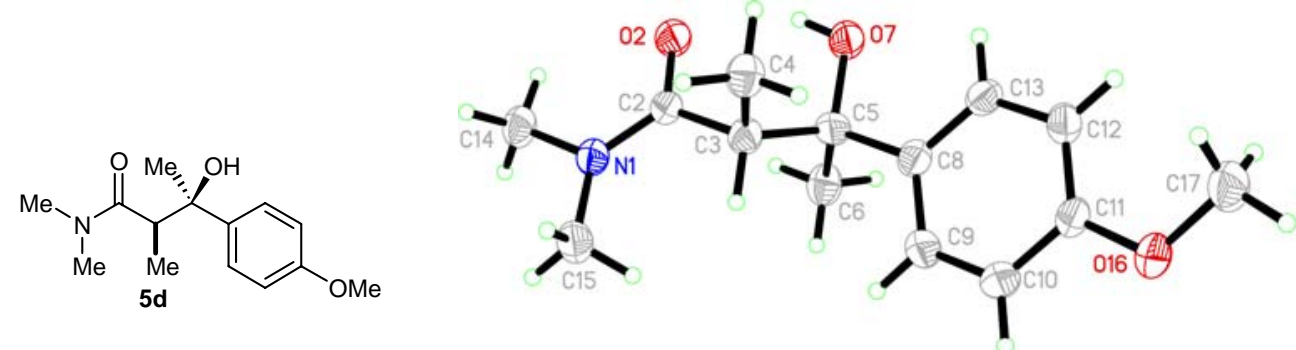

- The relative stereochemistry of $\mathbf{8 f}$ was determined by X-ray crystallography.
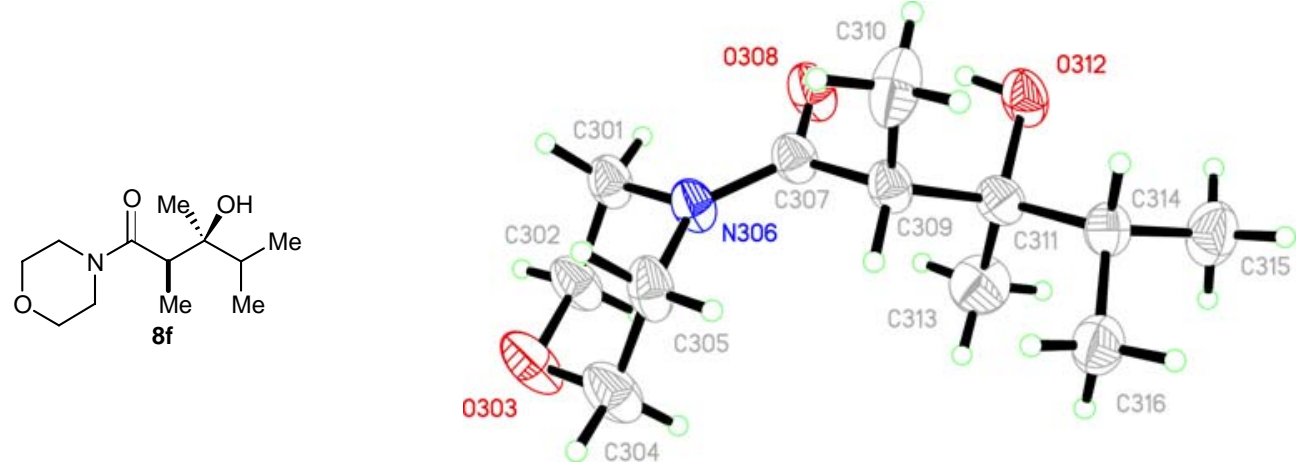

- $\quad$ The relative stereochemistry of $\mathbf{8 g}$ was determined by X-ray crystallography.
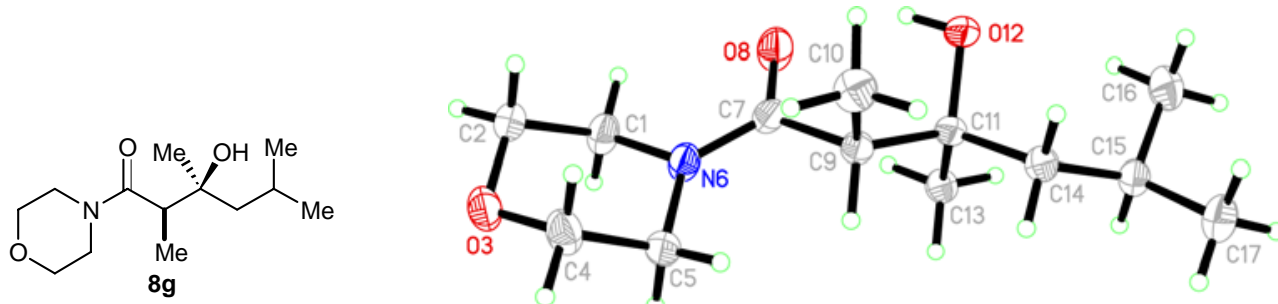

- The relative stereochemistries of the remaining products were assigned by analogy. 


\section{NMR Spectra}<smiles>O=C(/C=C/c1ccc2ccccc2c1)N1CCOCC1</smiles>

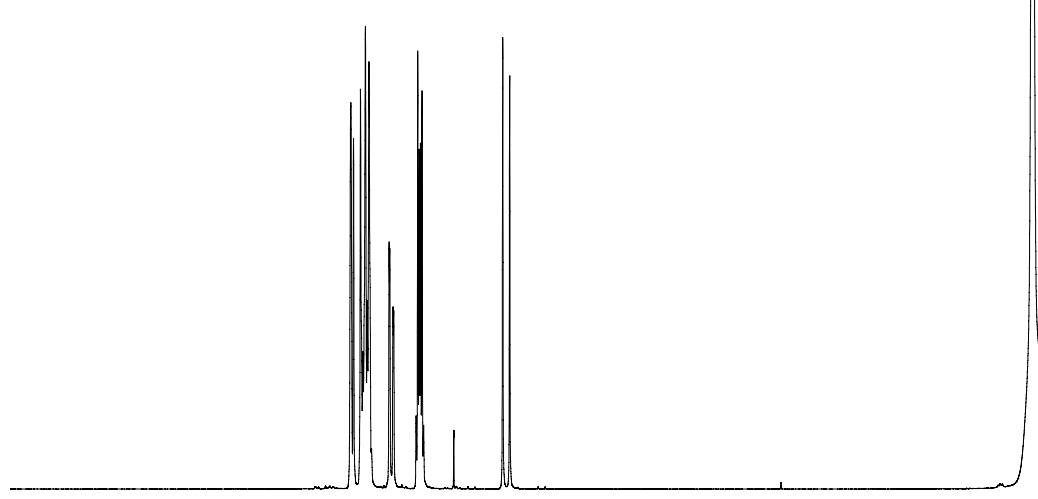

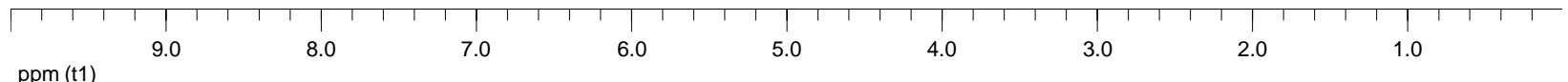

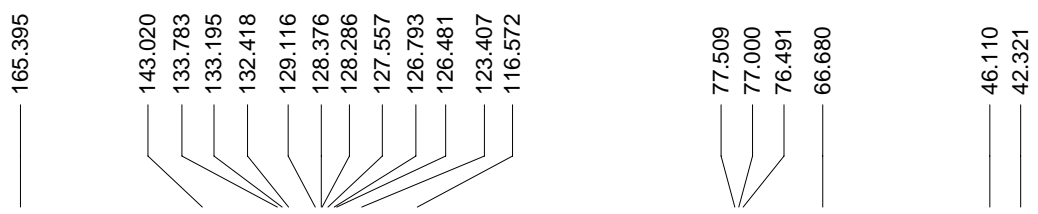

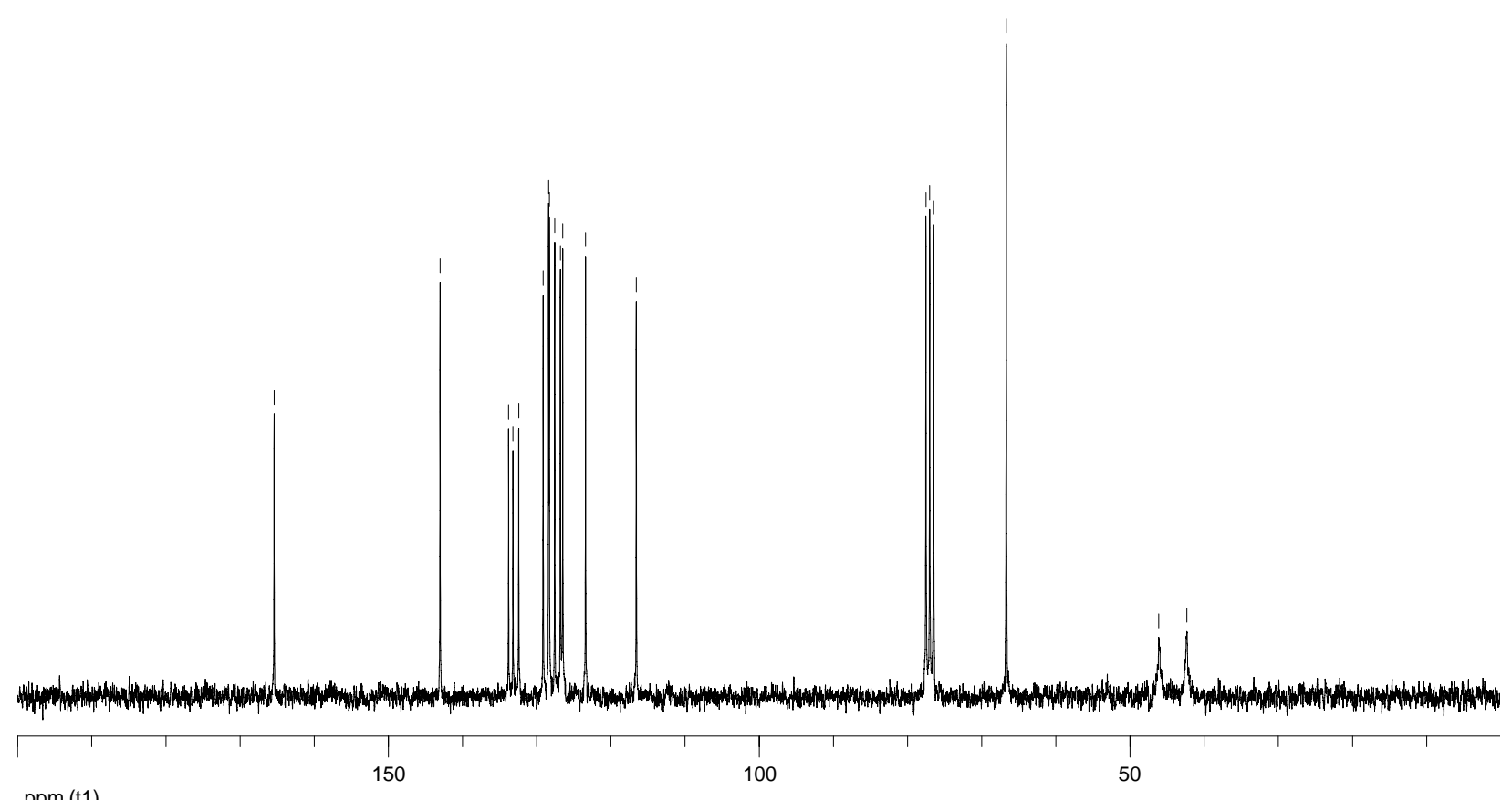


Supporting Information

S21
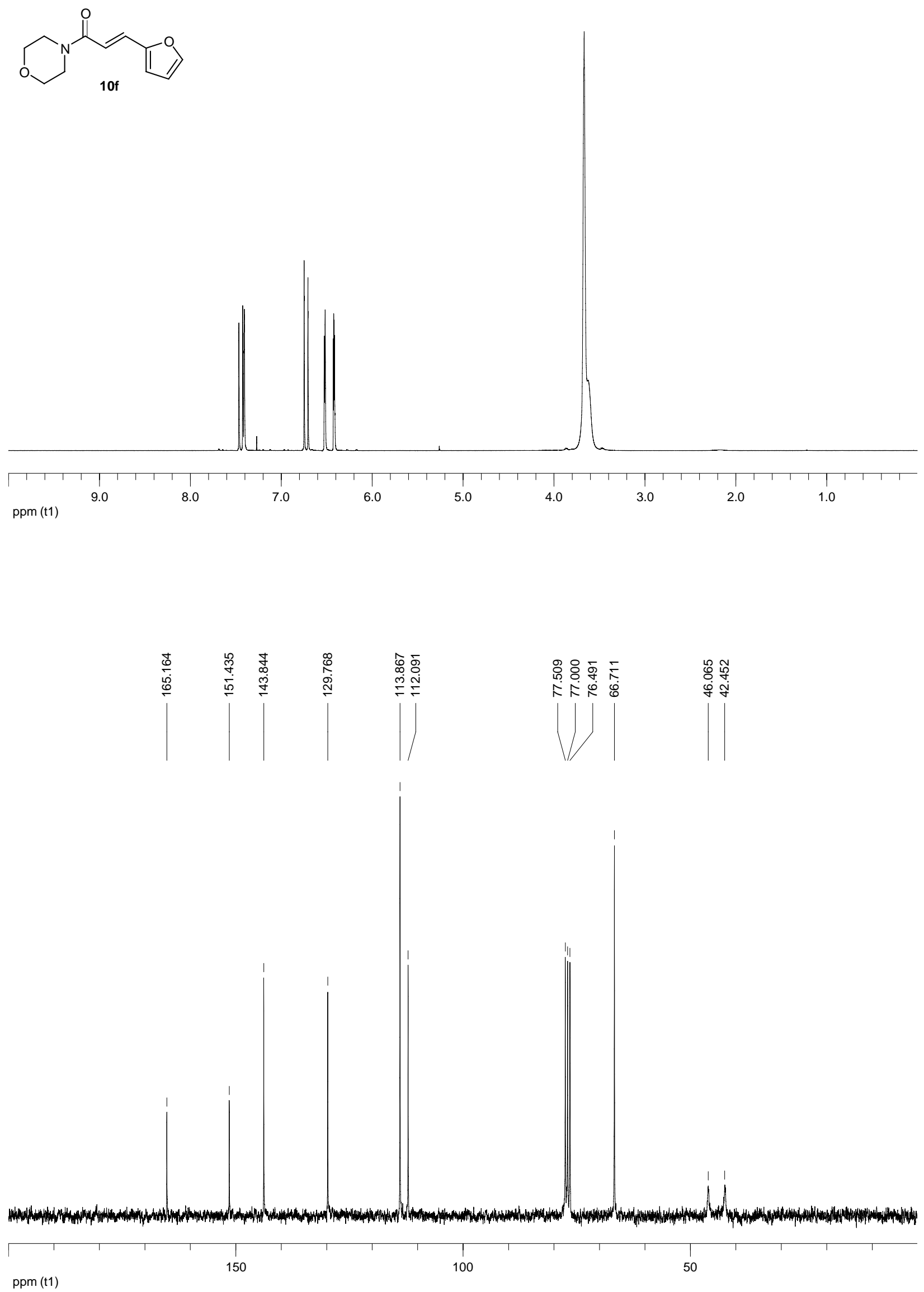

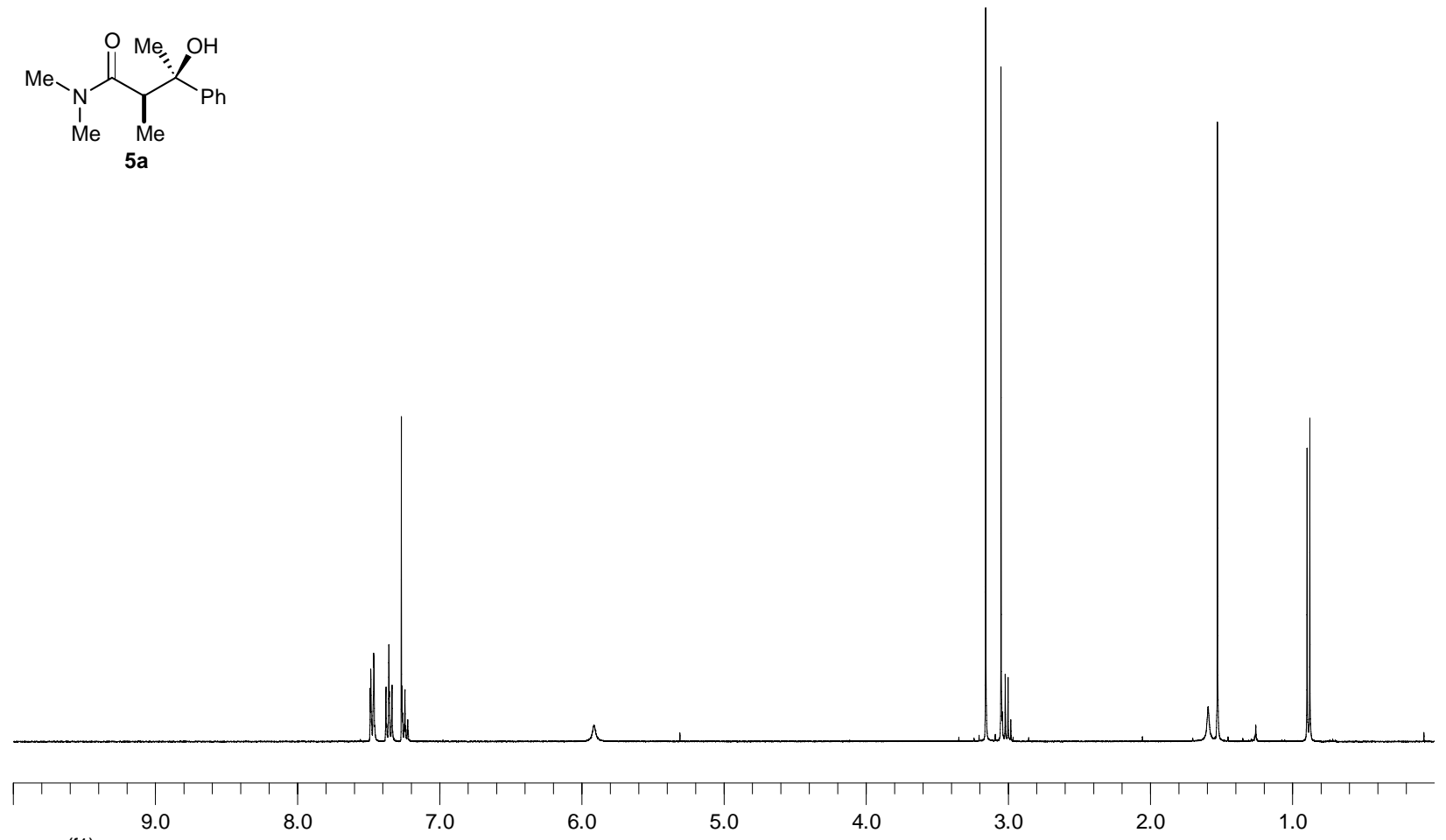

ppm (f1)

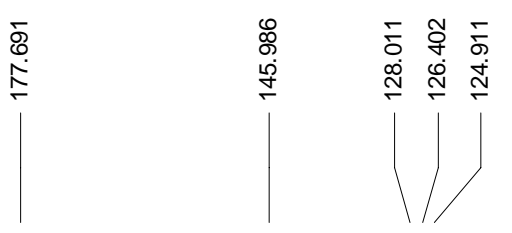
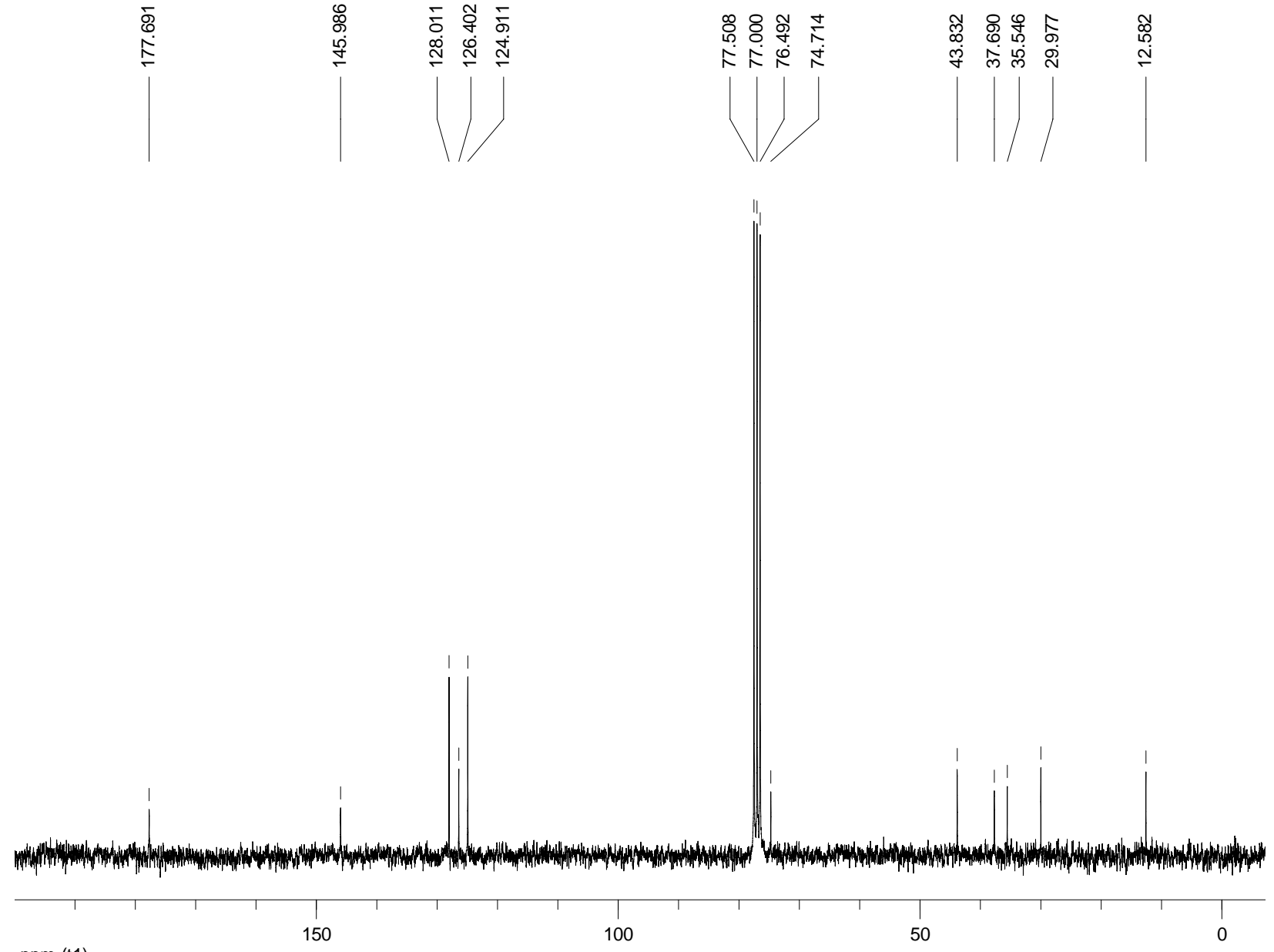

ppm (t1) 
Supporting Information

S23<smiles>Cc1ccccc1[C@@](C)(O)C(C)C(=O)N(C)C</smiles>
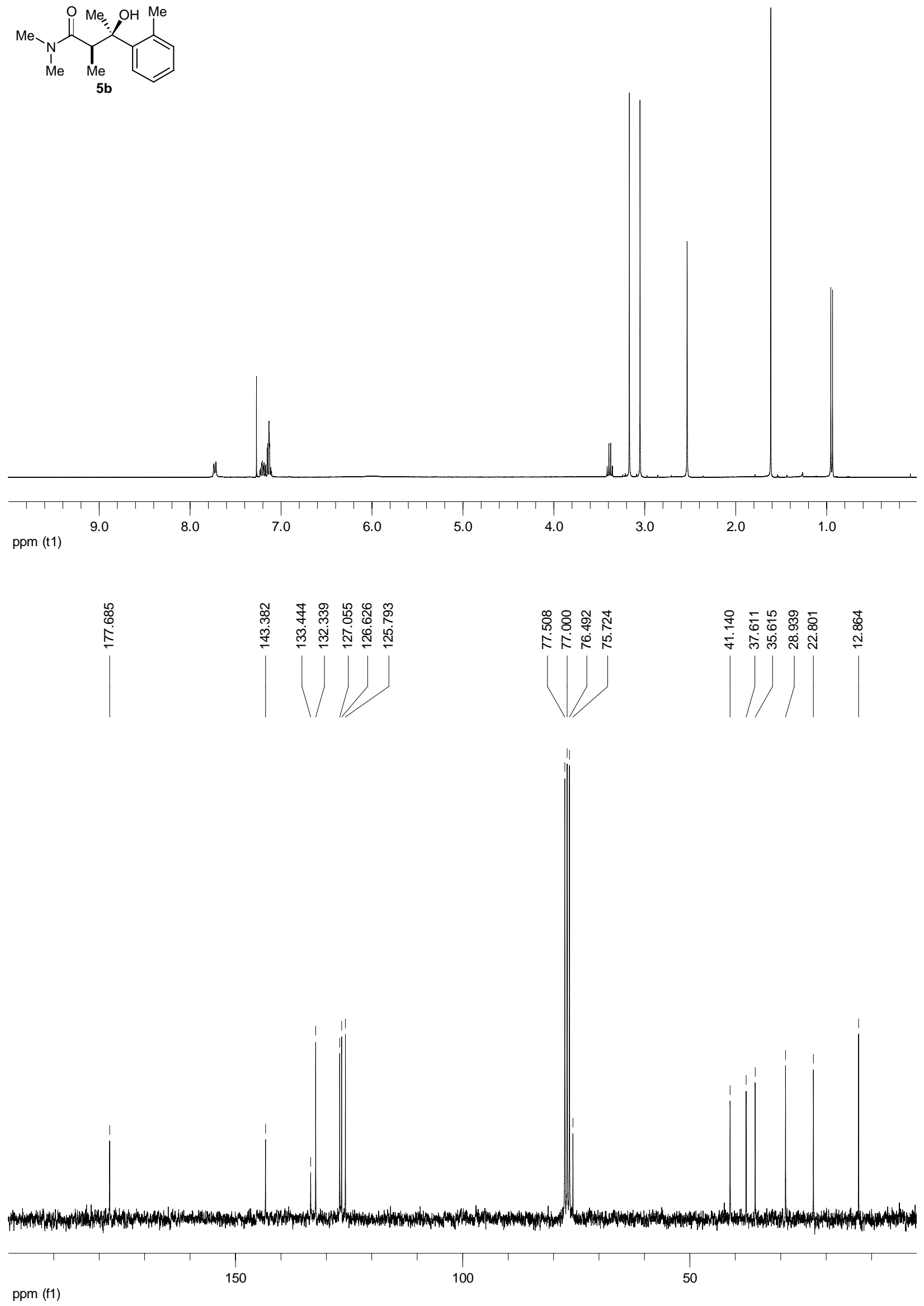

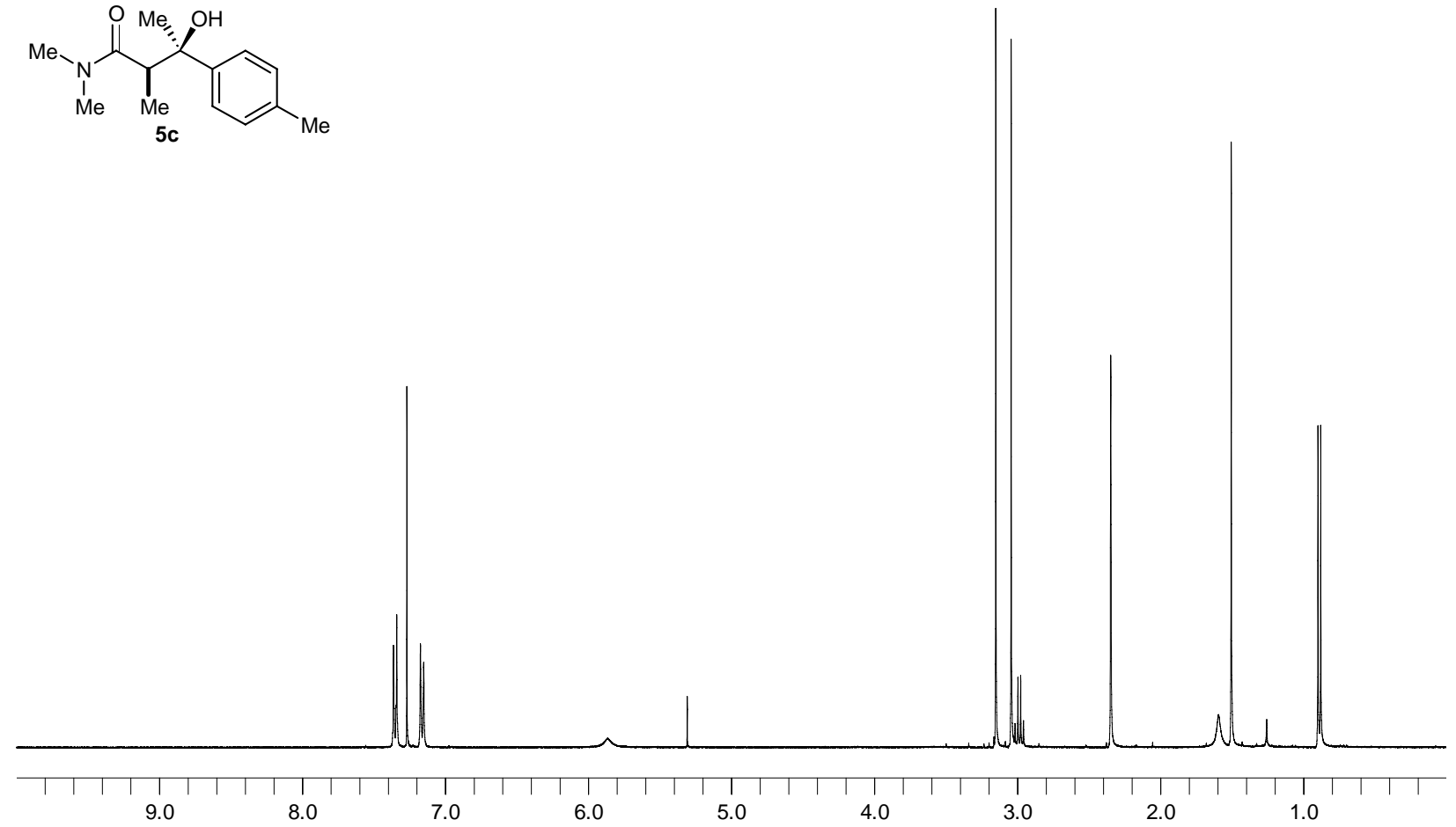

ppm (t1)
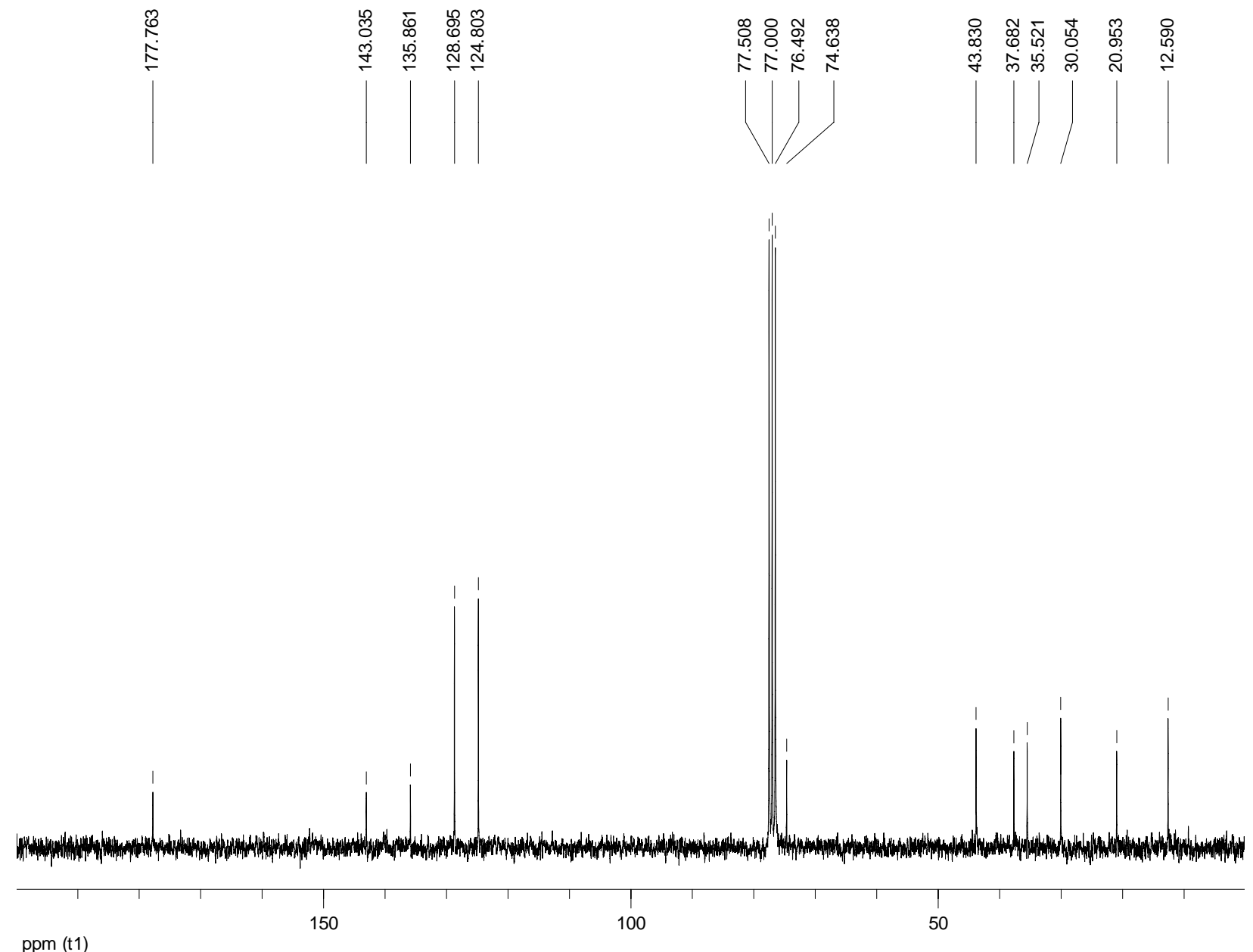

ppm (t1) 
Supporting Information

S25
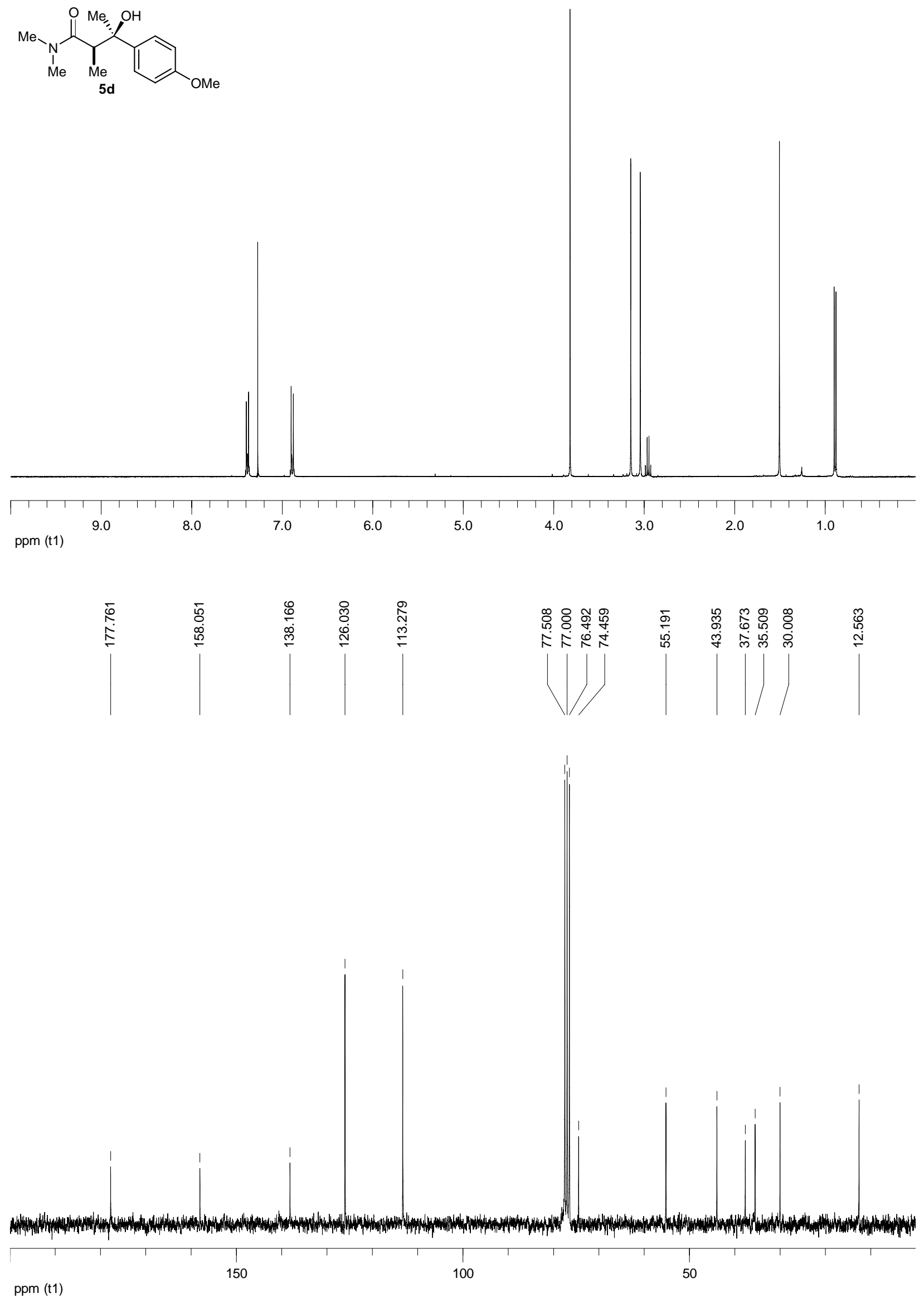

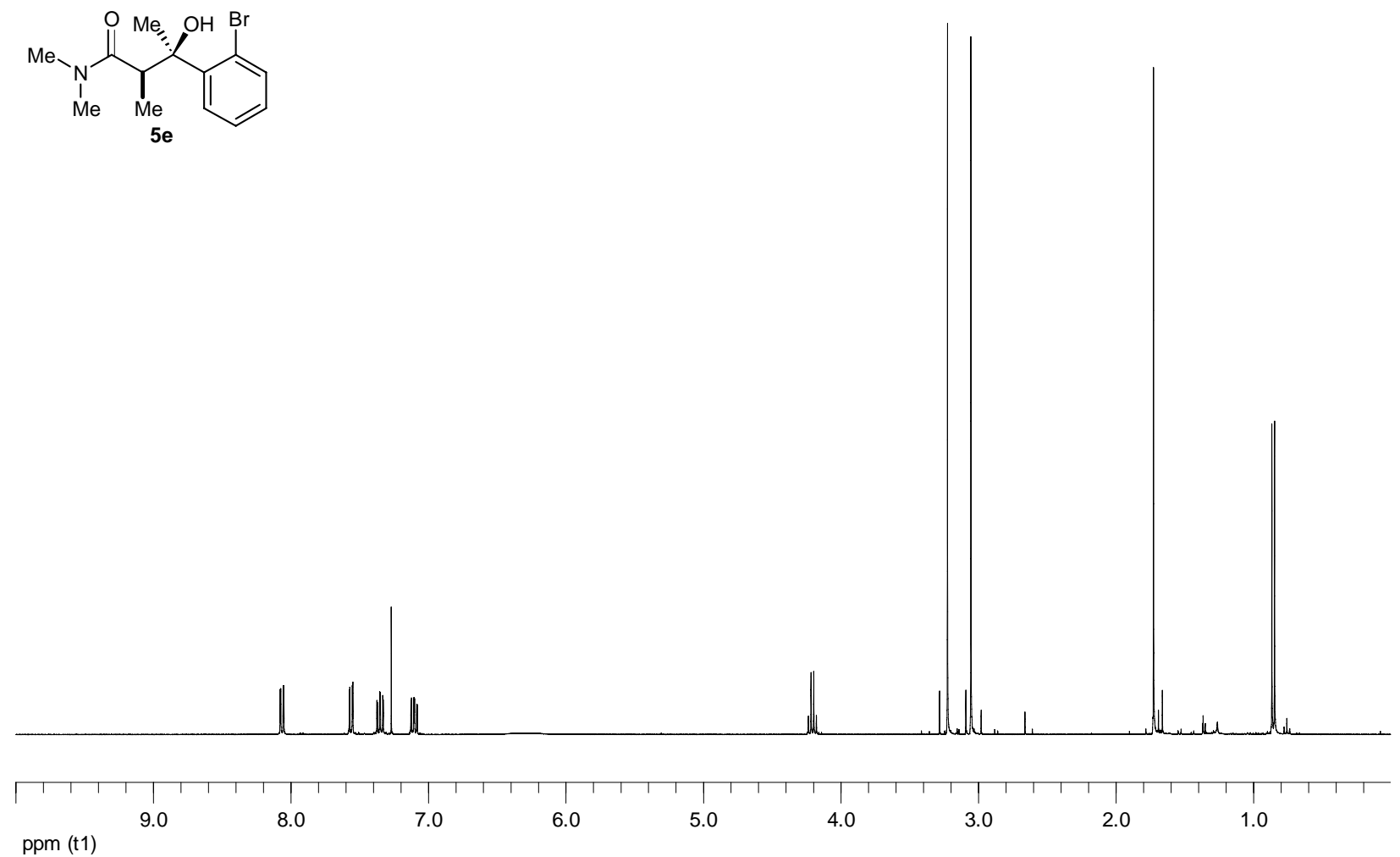

ppm (t1)
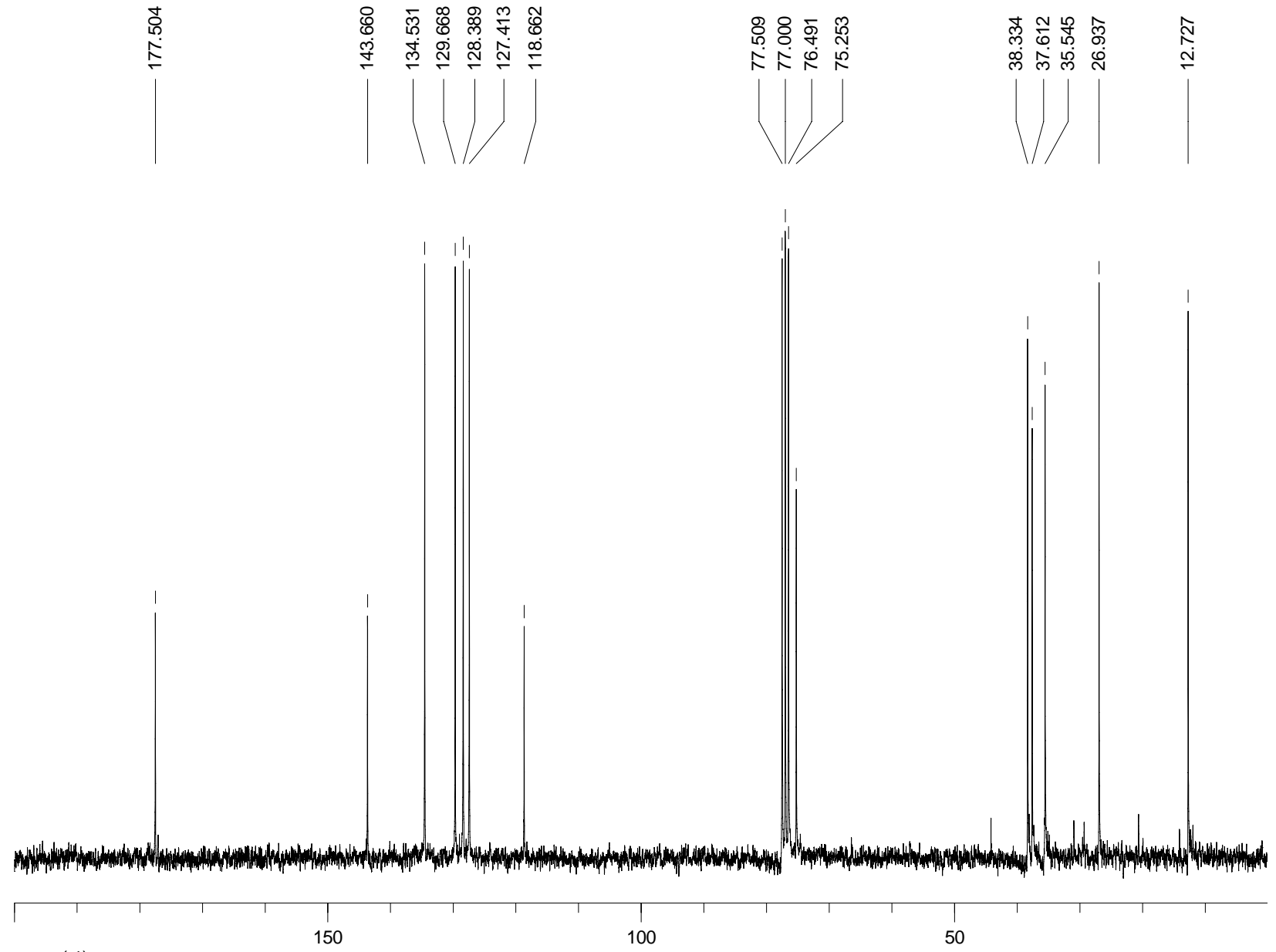

ppm (t1) 
Supporting Information

S27
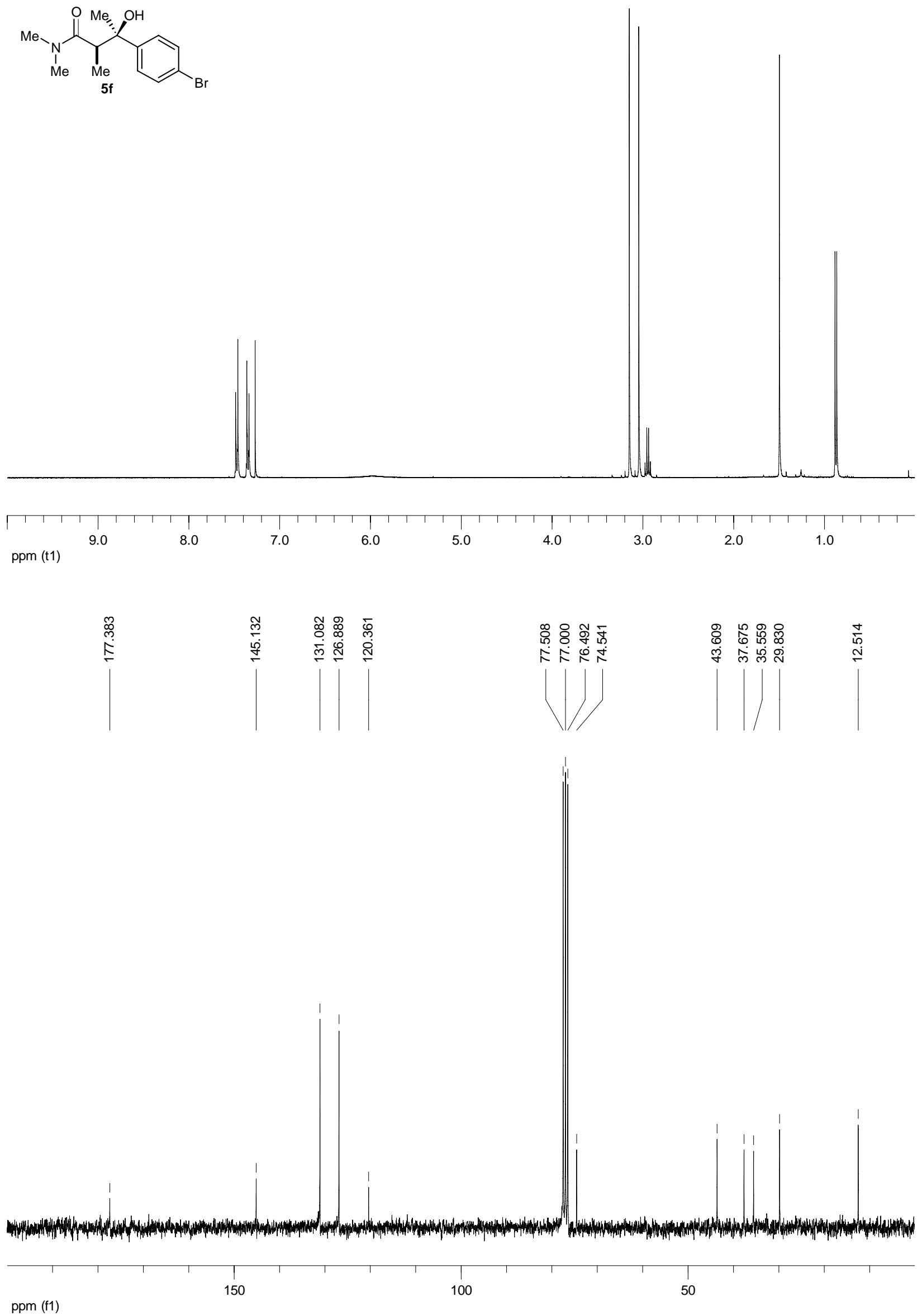
Supporting Information

S28
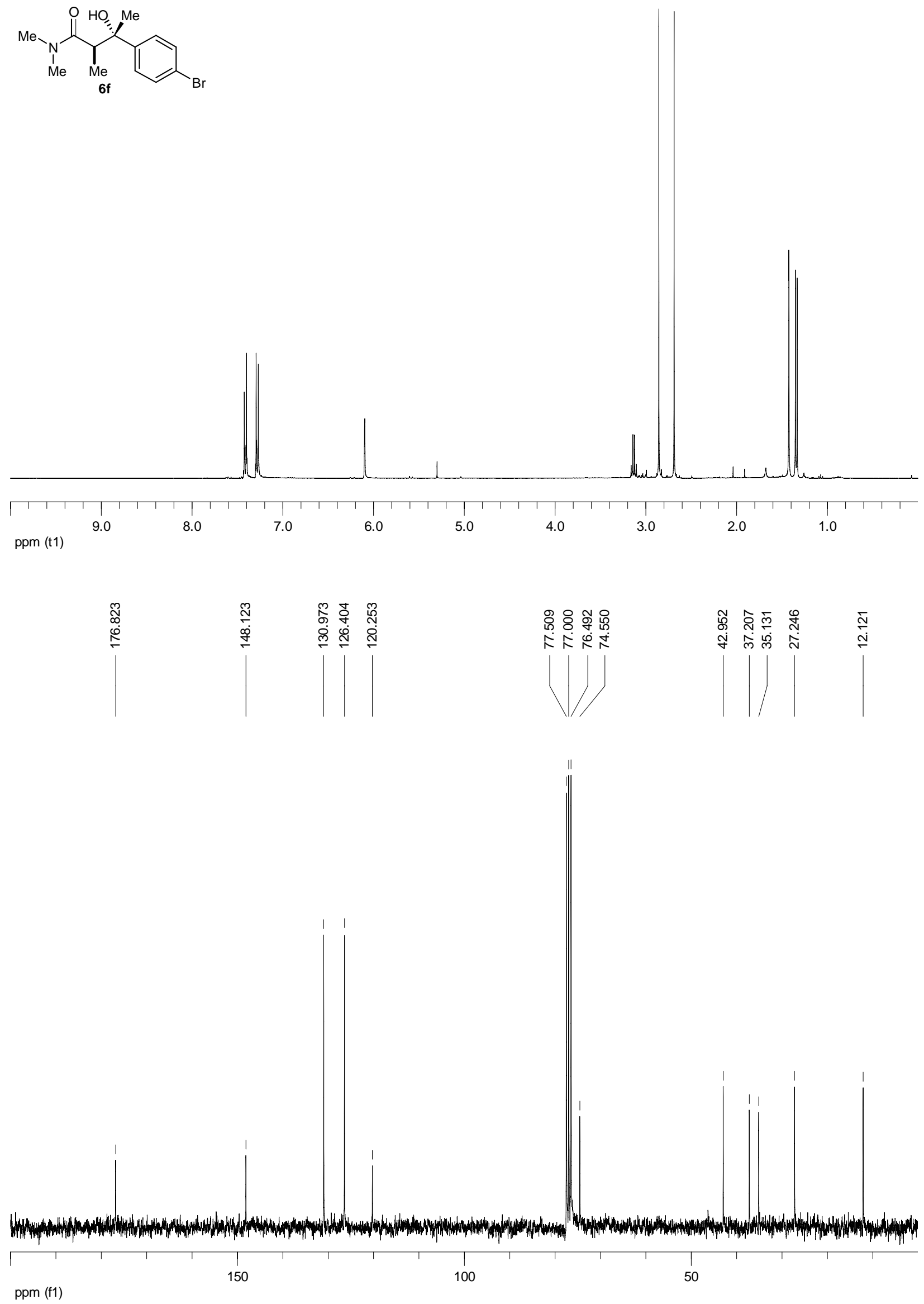

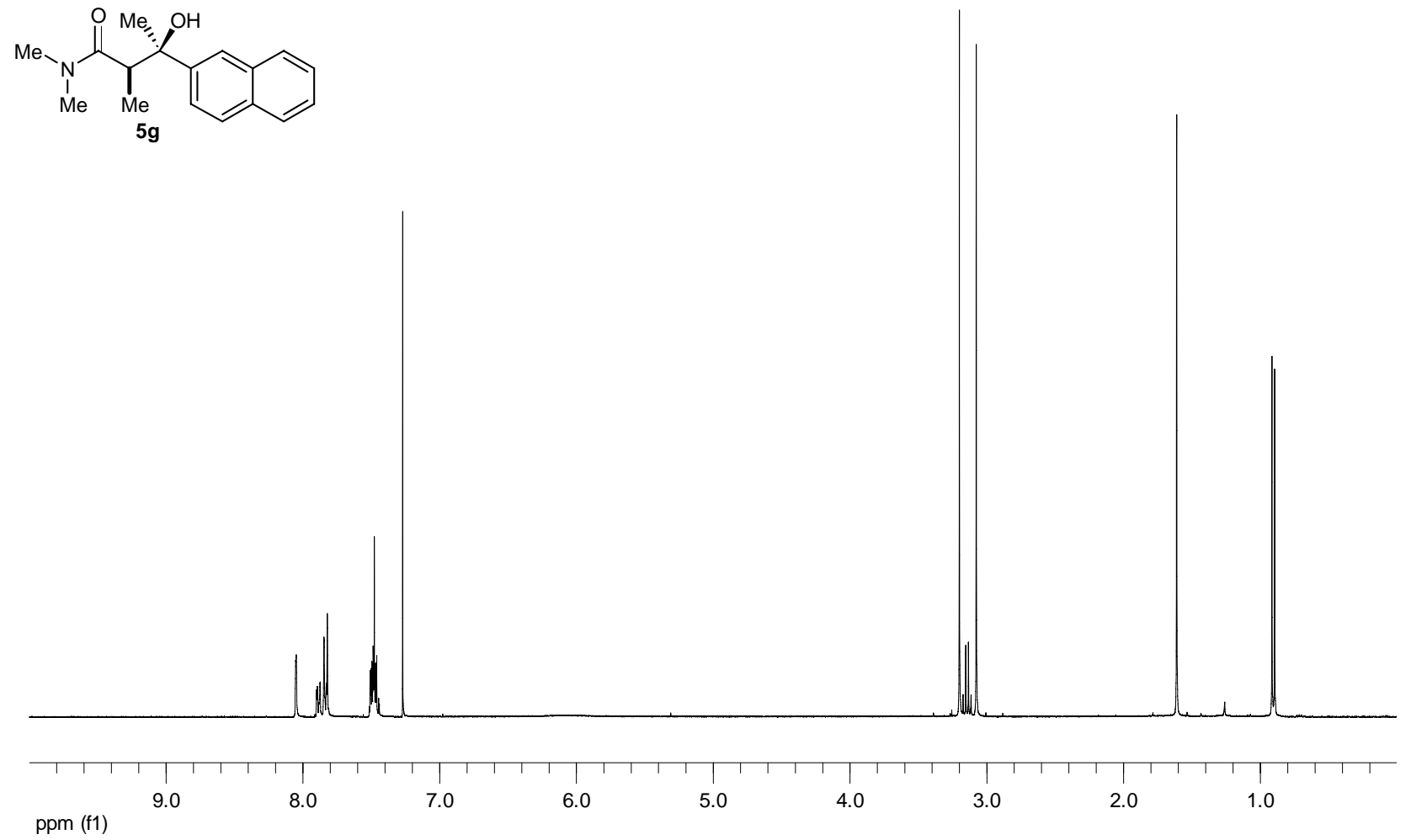

\&্ণ

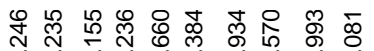

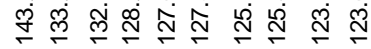

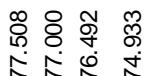

స్లి

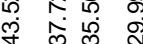

్ㅗㅇำ

స

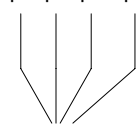

ल
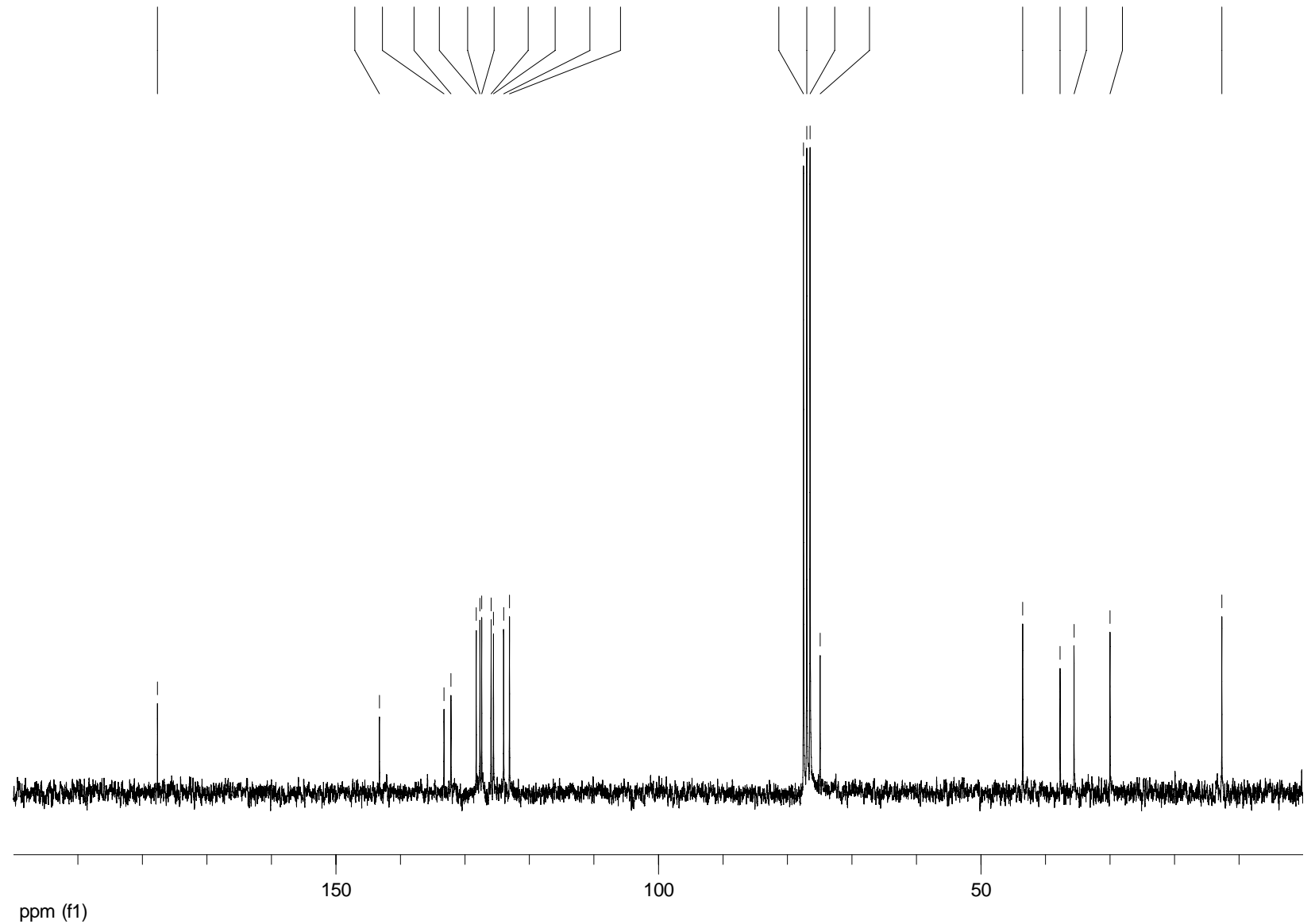

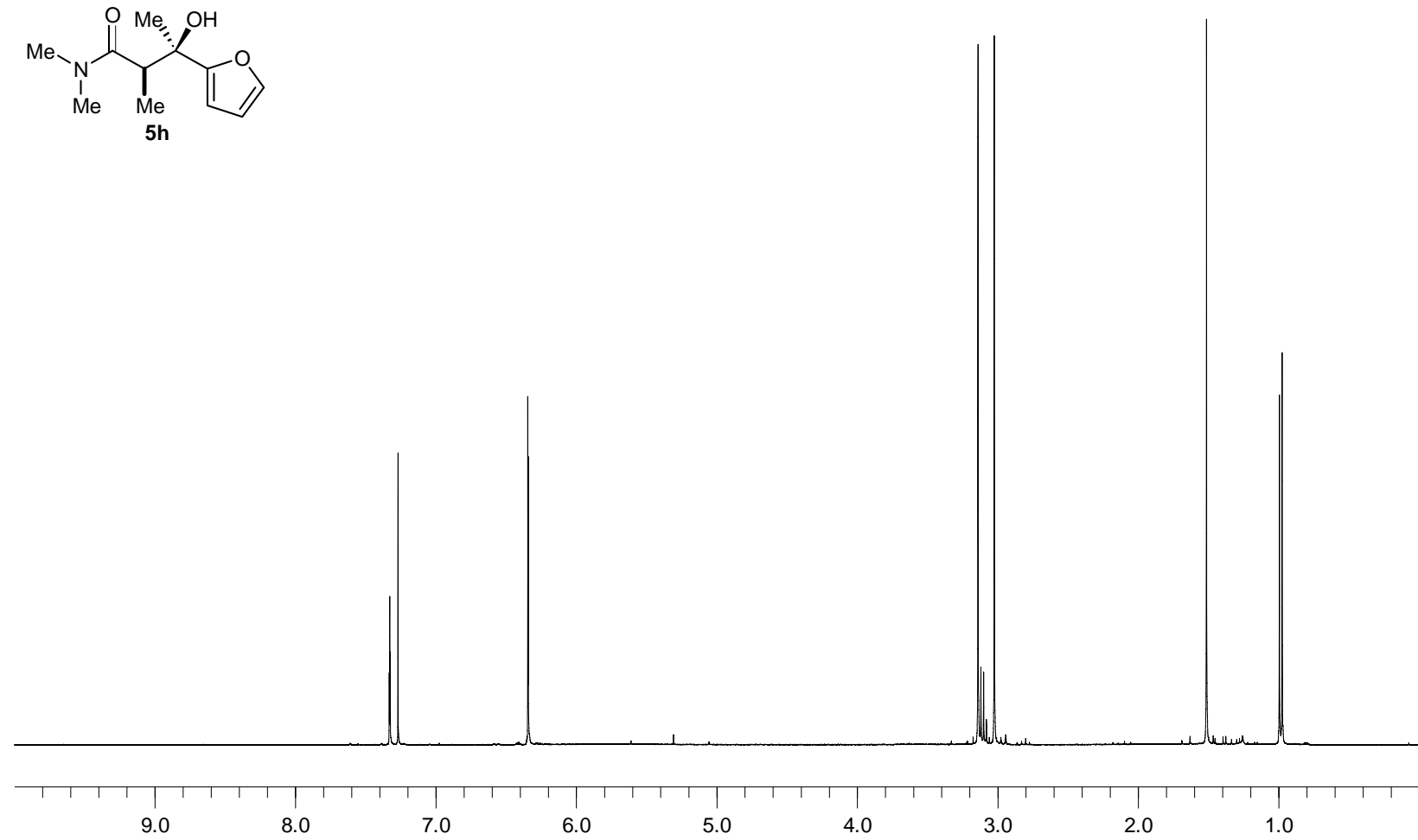

ppm (t1)

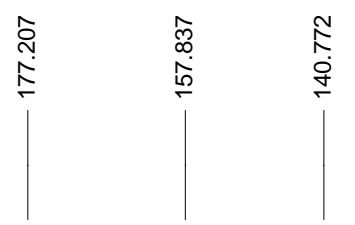

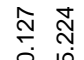

哭

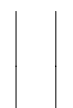

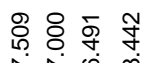

रト்̃

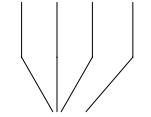

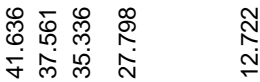

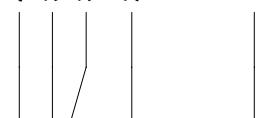

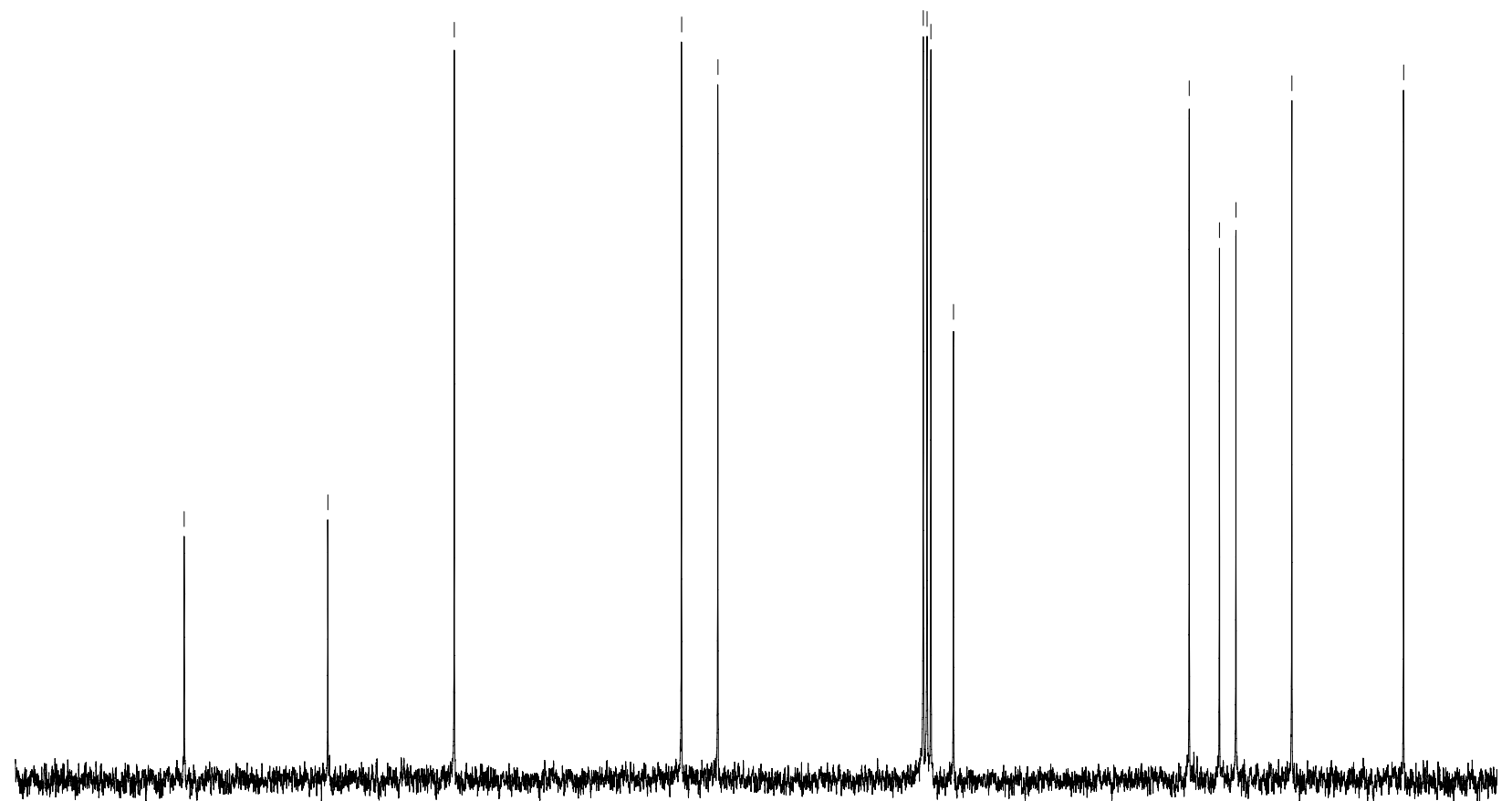

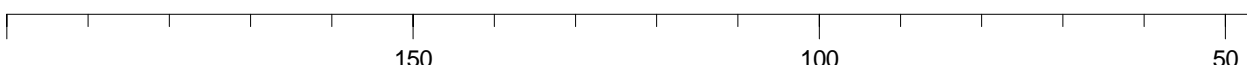

ppm (t1)

(5)


Supporting Information

S31<smiles>C[C@@H](O)C(C(=O)N(C)C)[C@@](C)(O)c1ccco1</smiles>
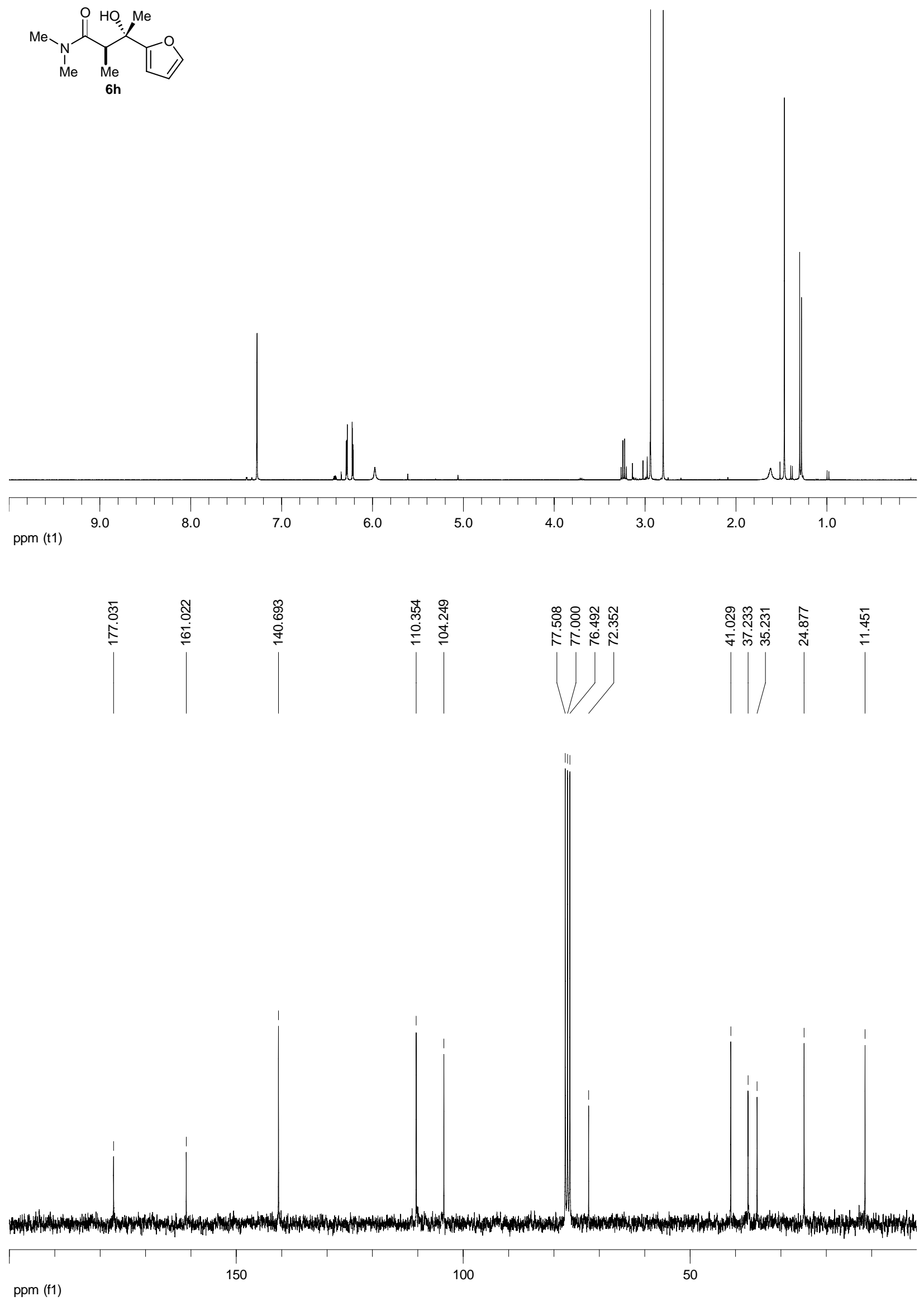


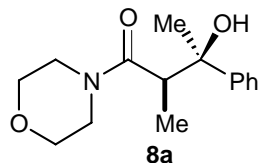

$8 \mathbf{a}$

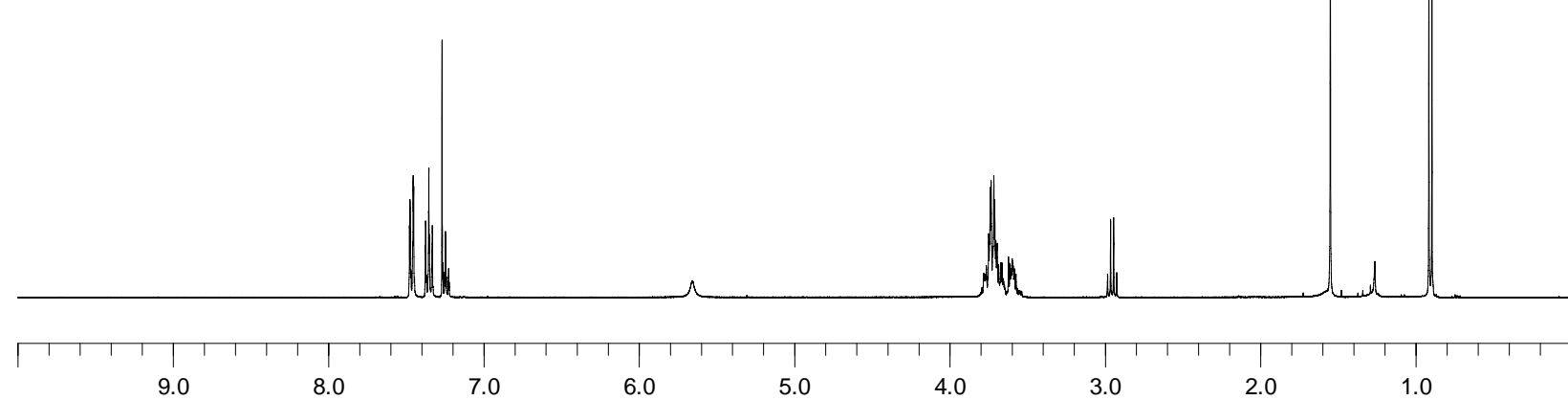

ppm (t1)

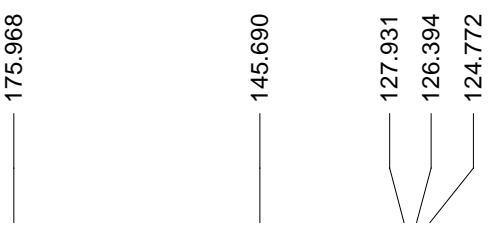
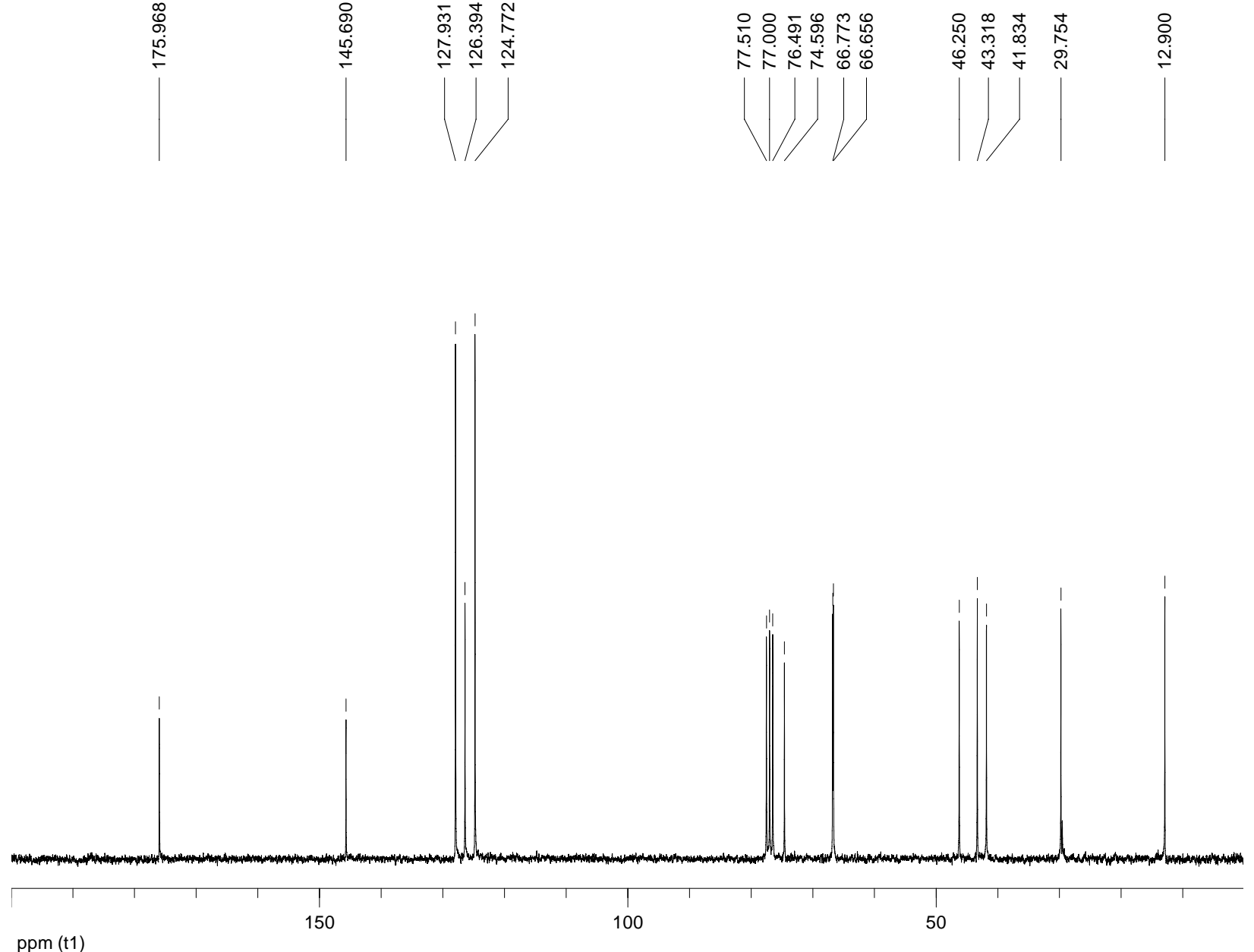
Supporting Information

S33<smiles>Cc1ccccc1[C@](C)(O)C(C)C(=O)N1CCOCC1</smiles>

$8 b$
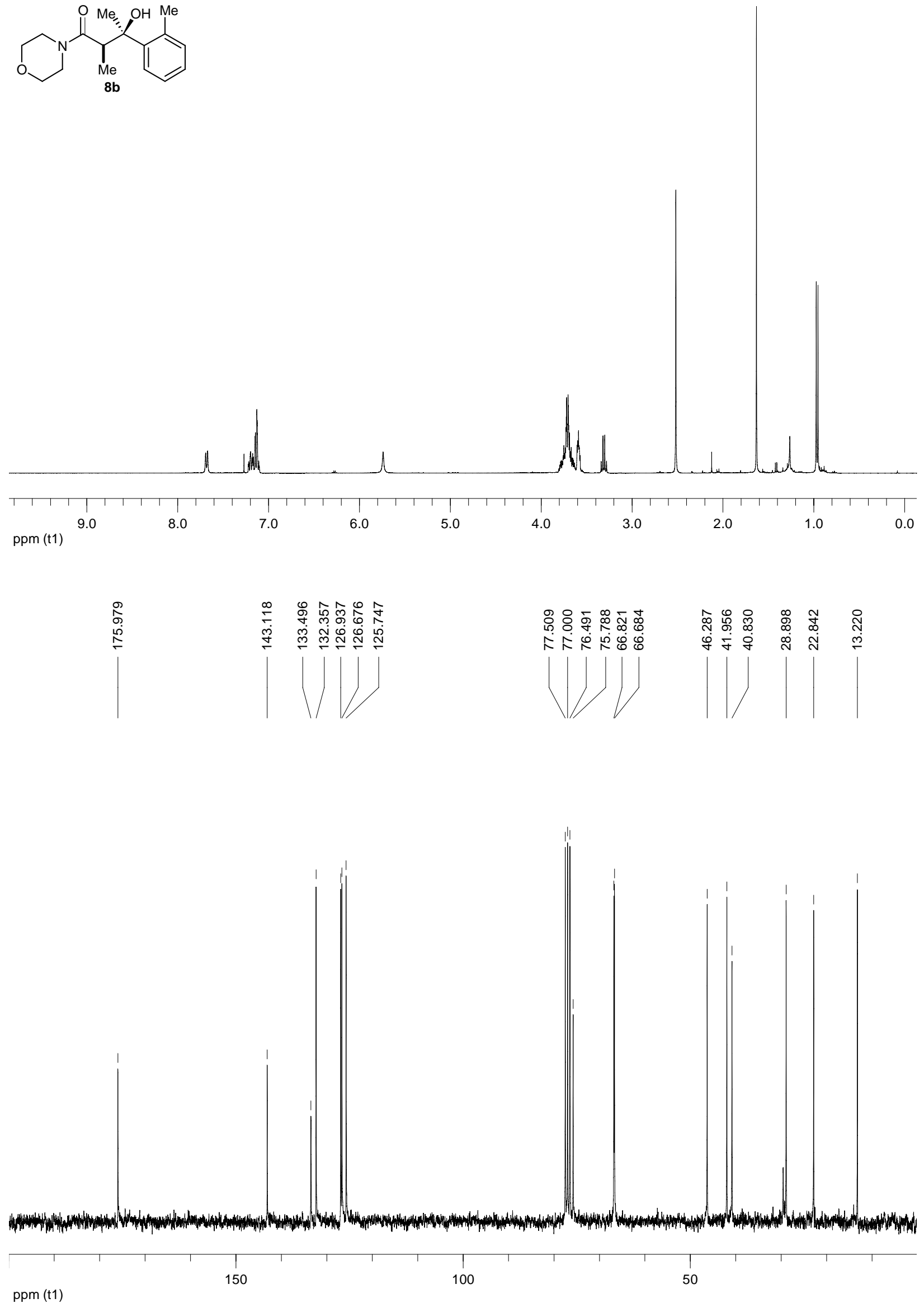
Supporting Information

S34
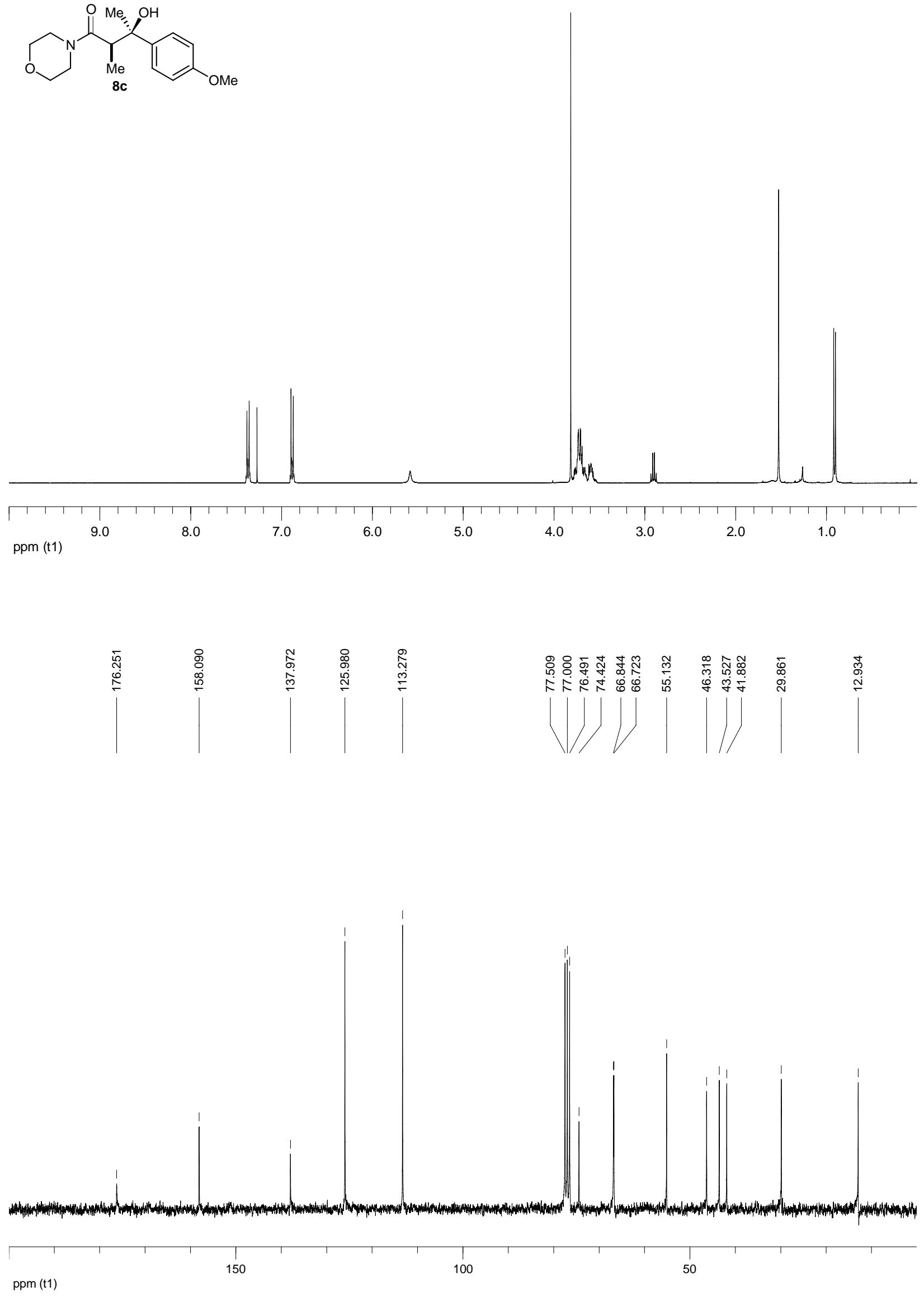
<smiles>CC(C(=O)N1CCOCC1)[C@@](C)(O)c1ccc2ccccc2c1</smiles>

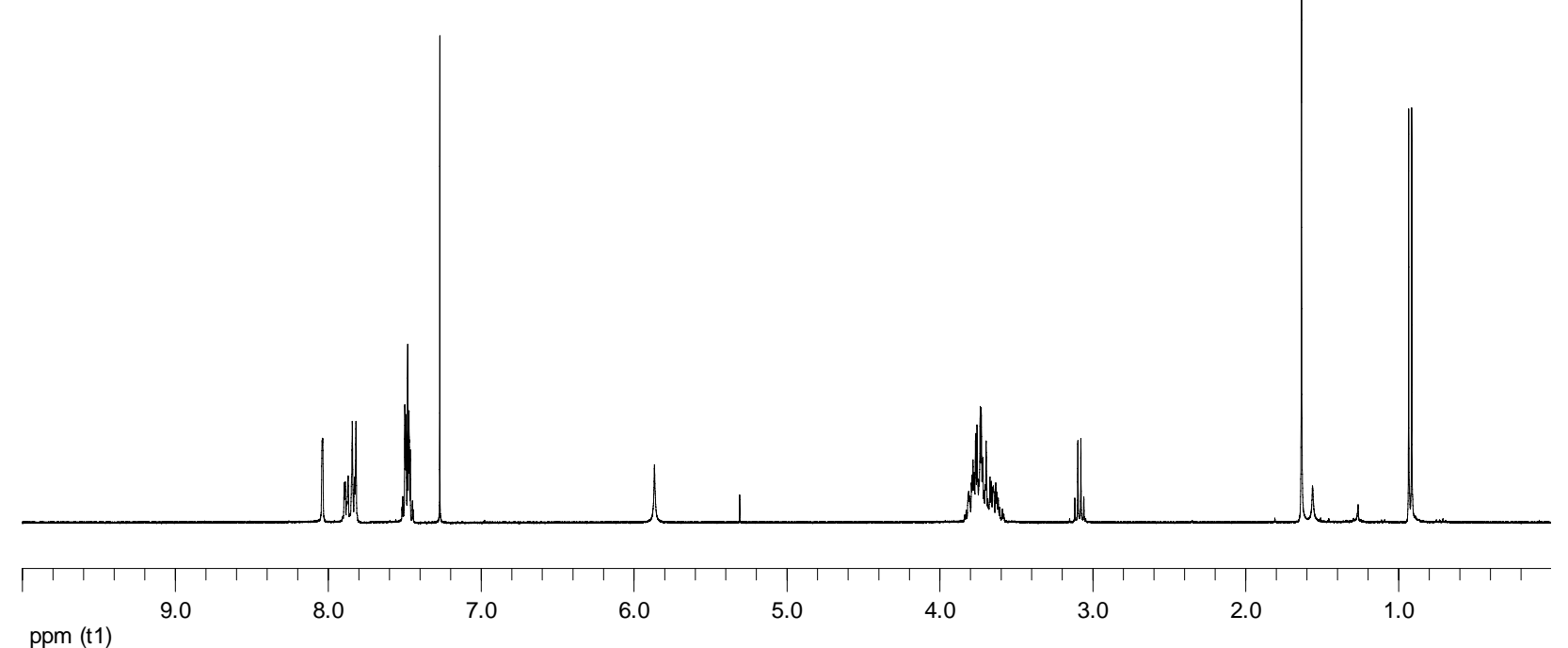

ppm (t1)

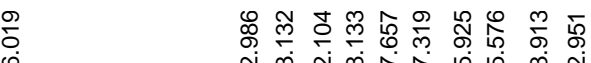

๖ำ

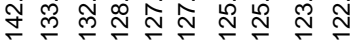
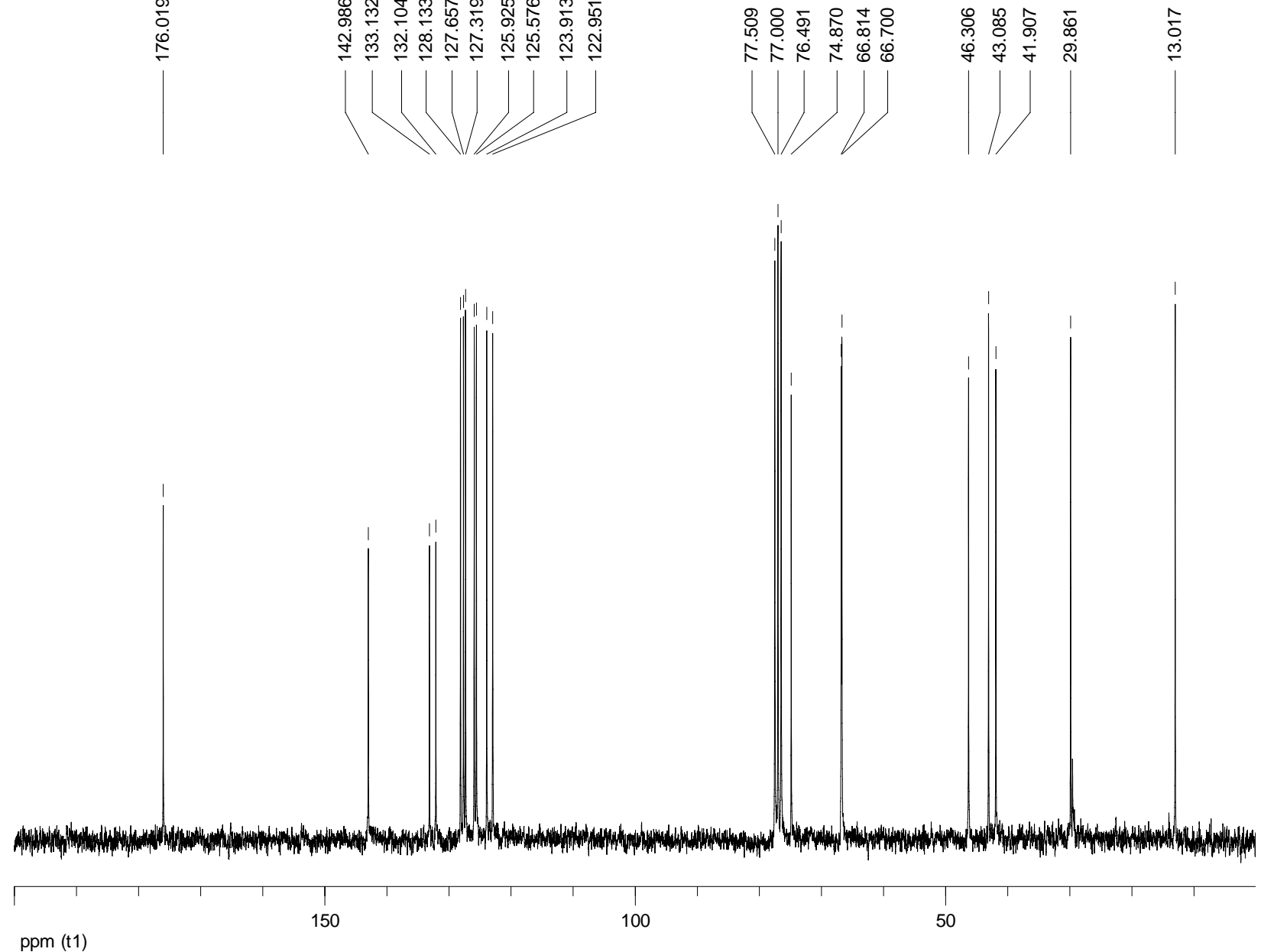
<smiles>CC(C(=O)N1CCOCC1)[C@@](C)(O)c1ccc2ccccc2c1</smiles>
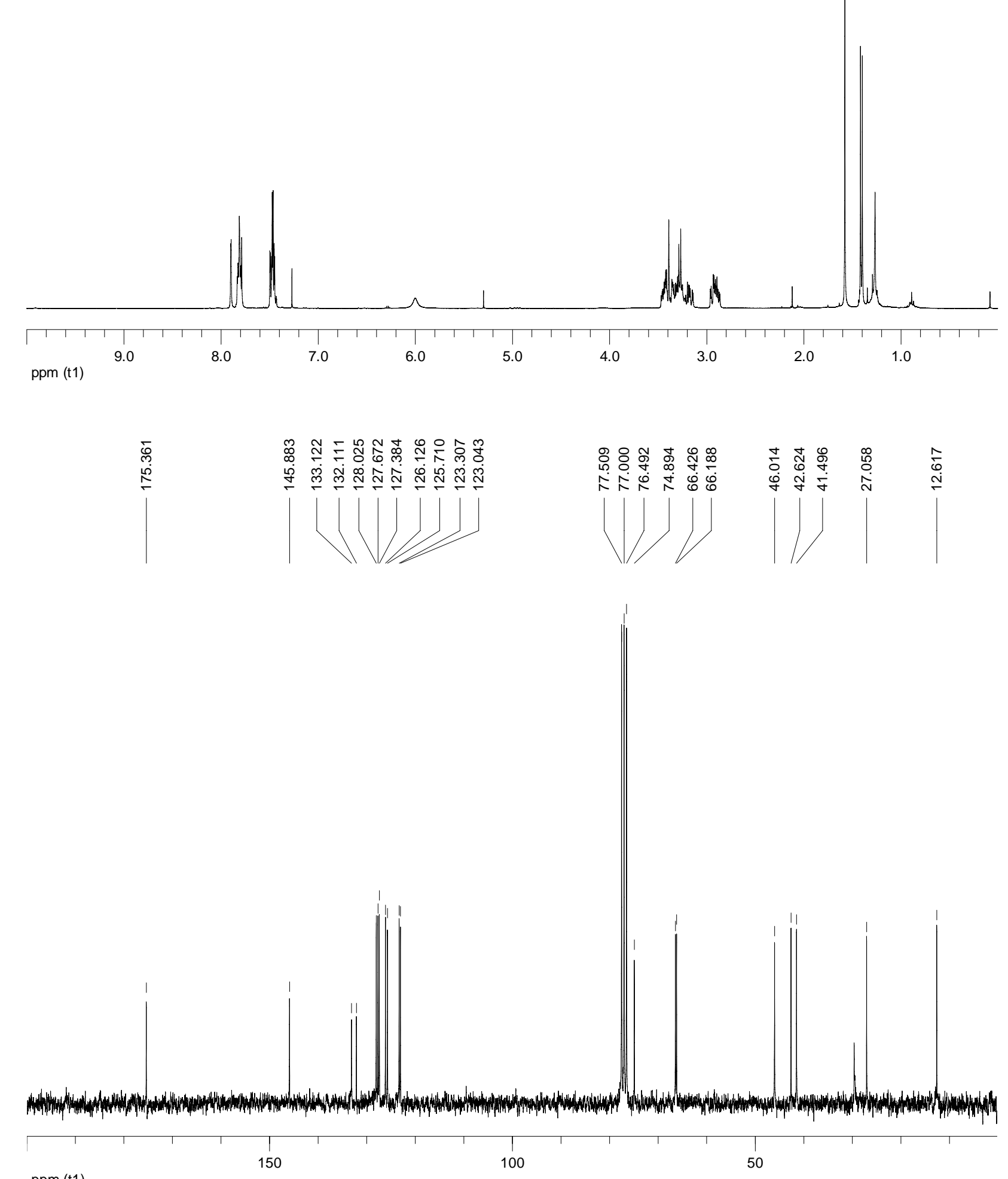

ppm (t1) 
<smiles>CC(C(=O)N1CCOCC1)[C@@](C)(O)c1ccco1</smiles>

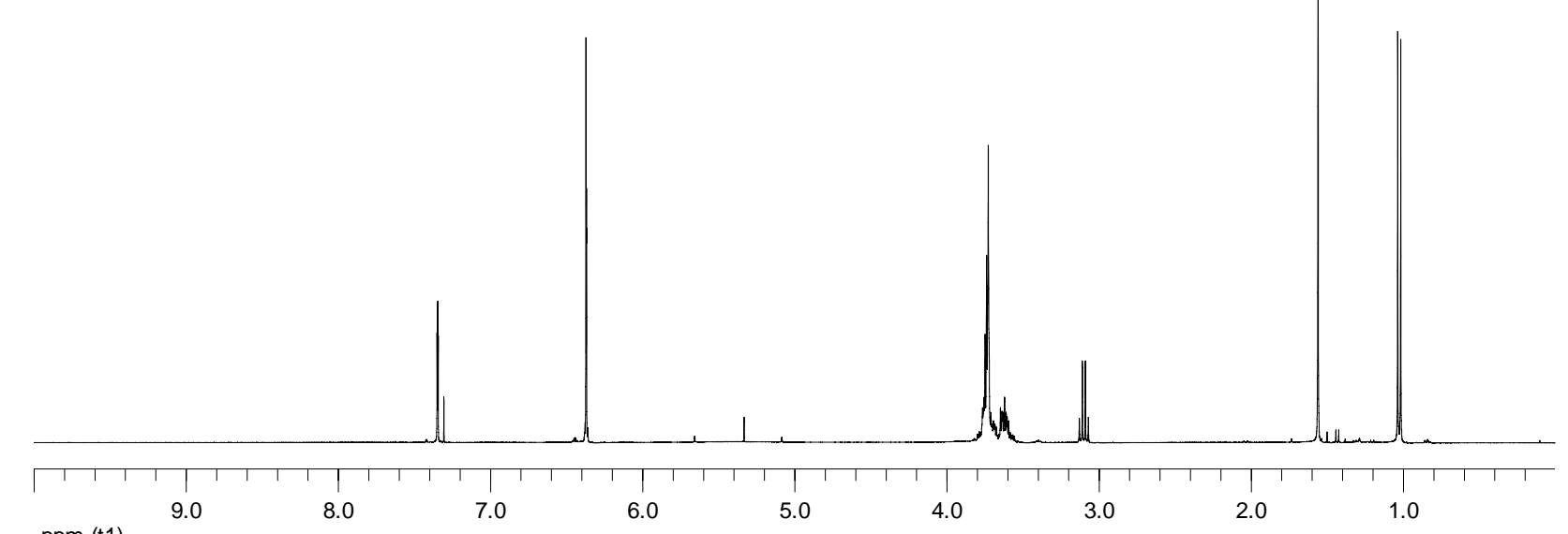

ppm (t1)
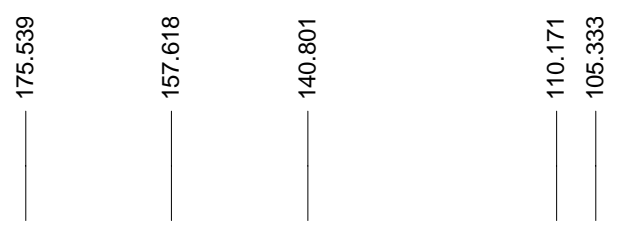

웅요웡

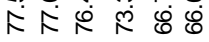

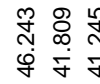
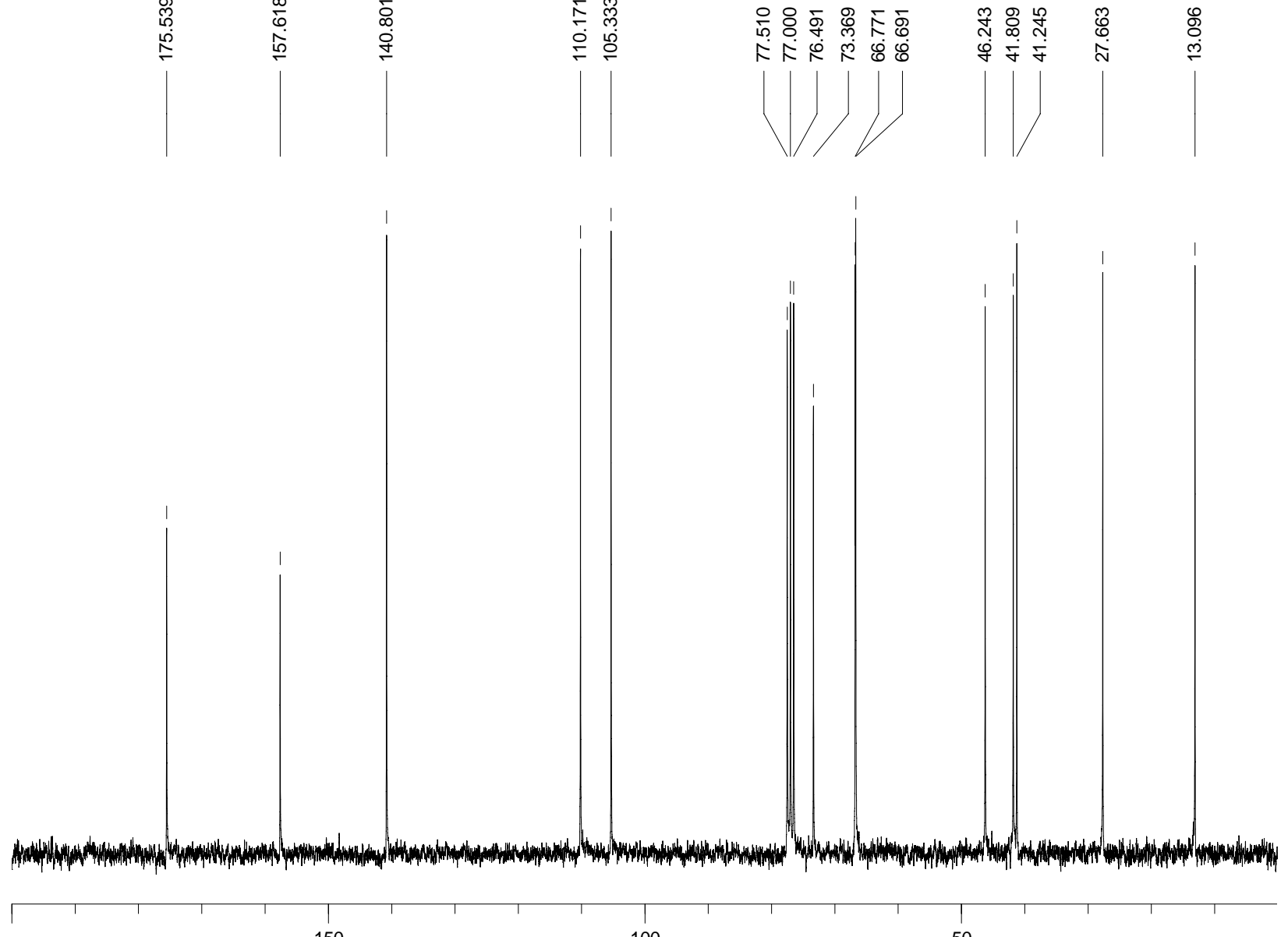

ppm (t1)

150

100

50 

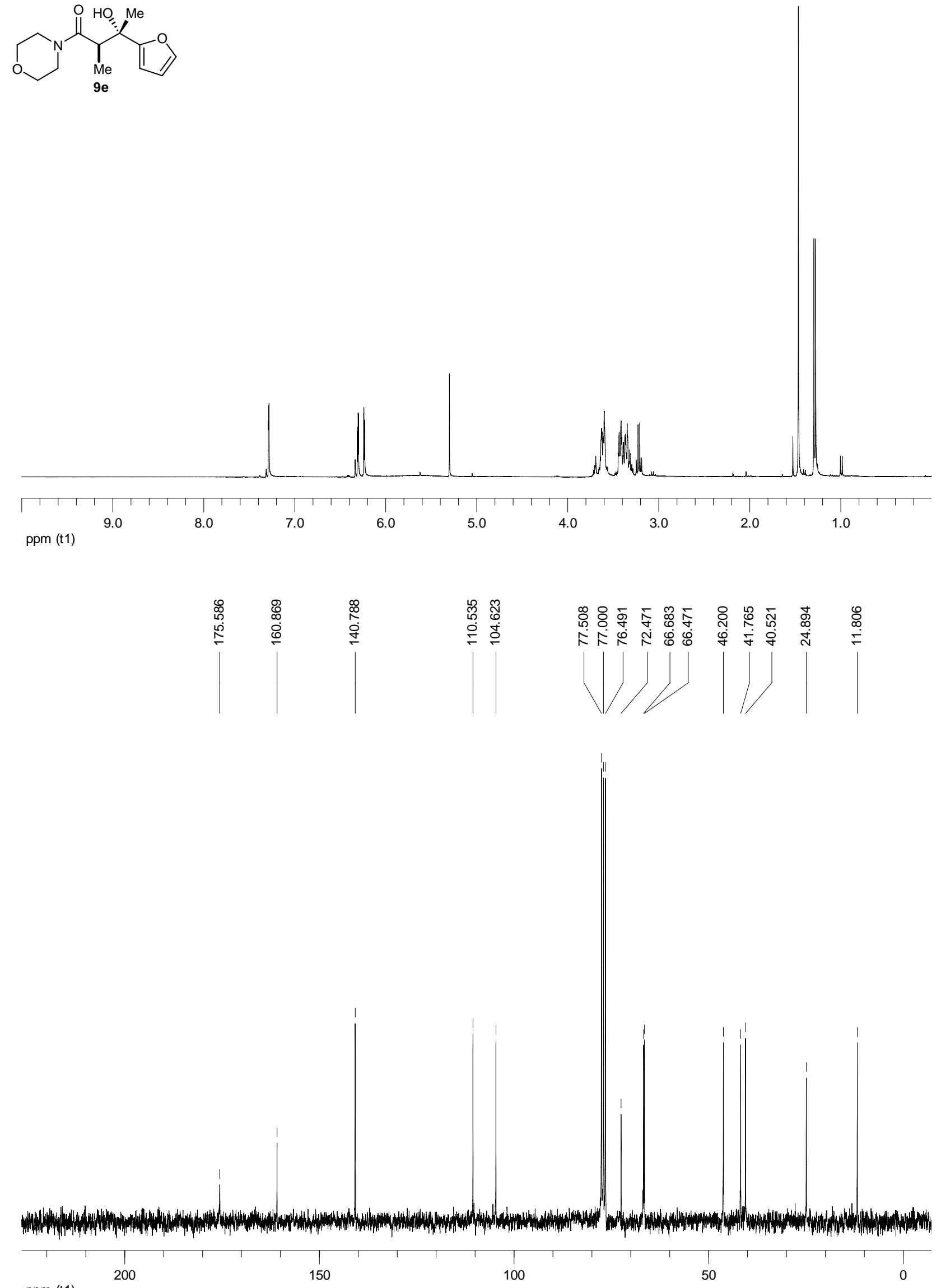

ppm (t1) 
<smiles>CC(C)C(C)(O)C(C)C(=O)N1CCOCC1</smiles>

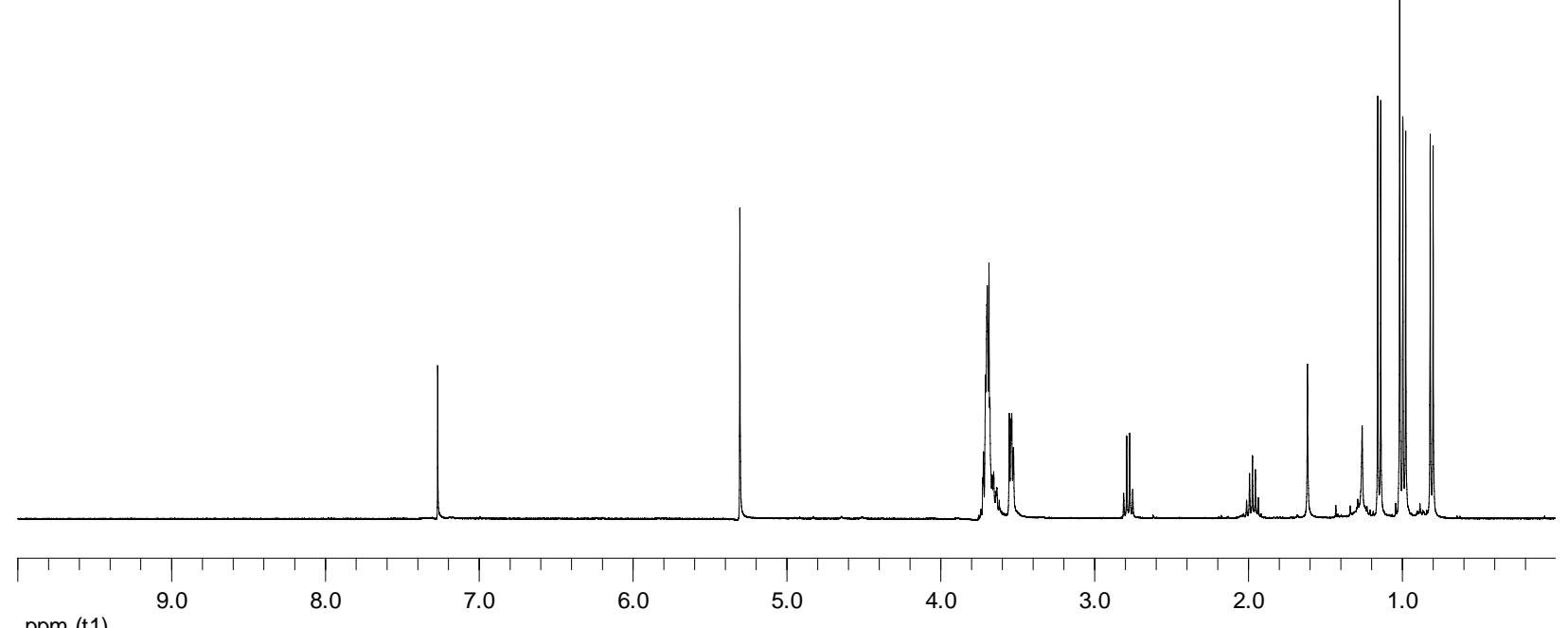
ppm (t1)
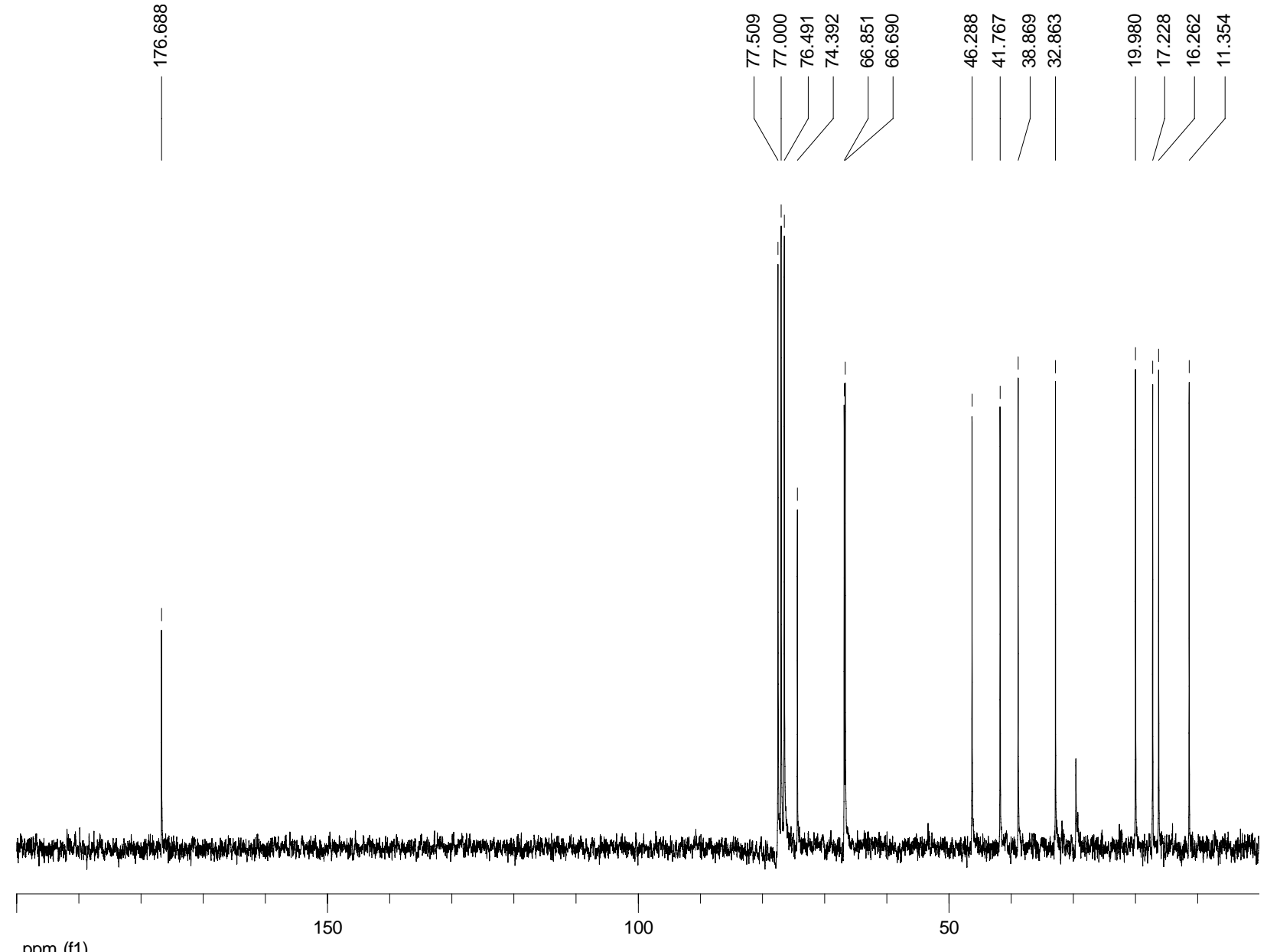

ppm (f1) 
<smiles>CC(C)[C@@](C)(O)C(C)C(=O)N1CCOCC1</smiles>

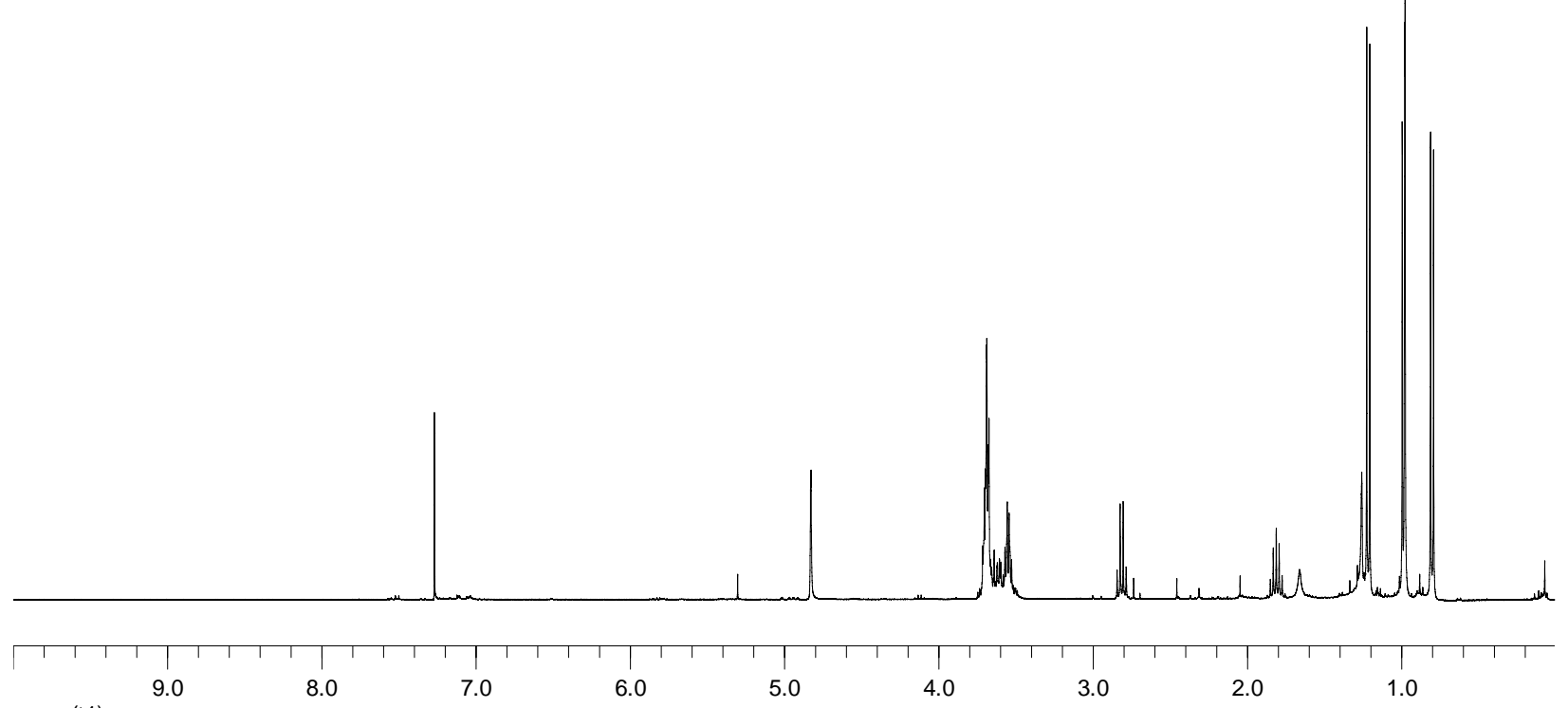

ppm (t1)

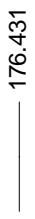

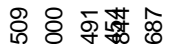

ト ト ம்i

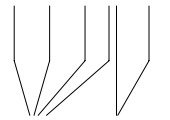

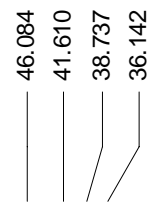

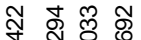

品 సี

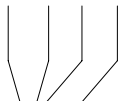

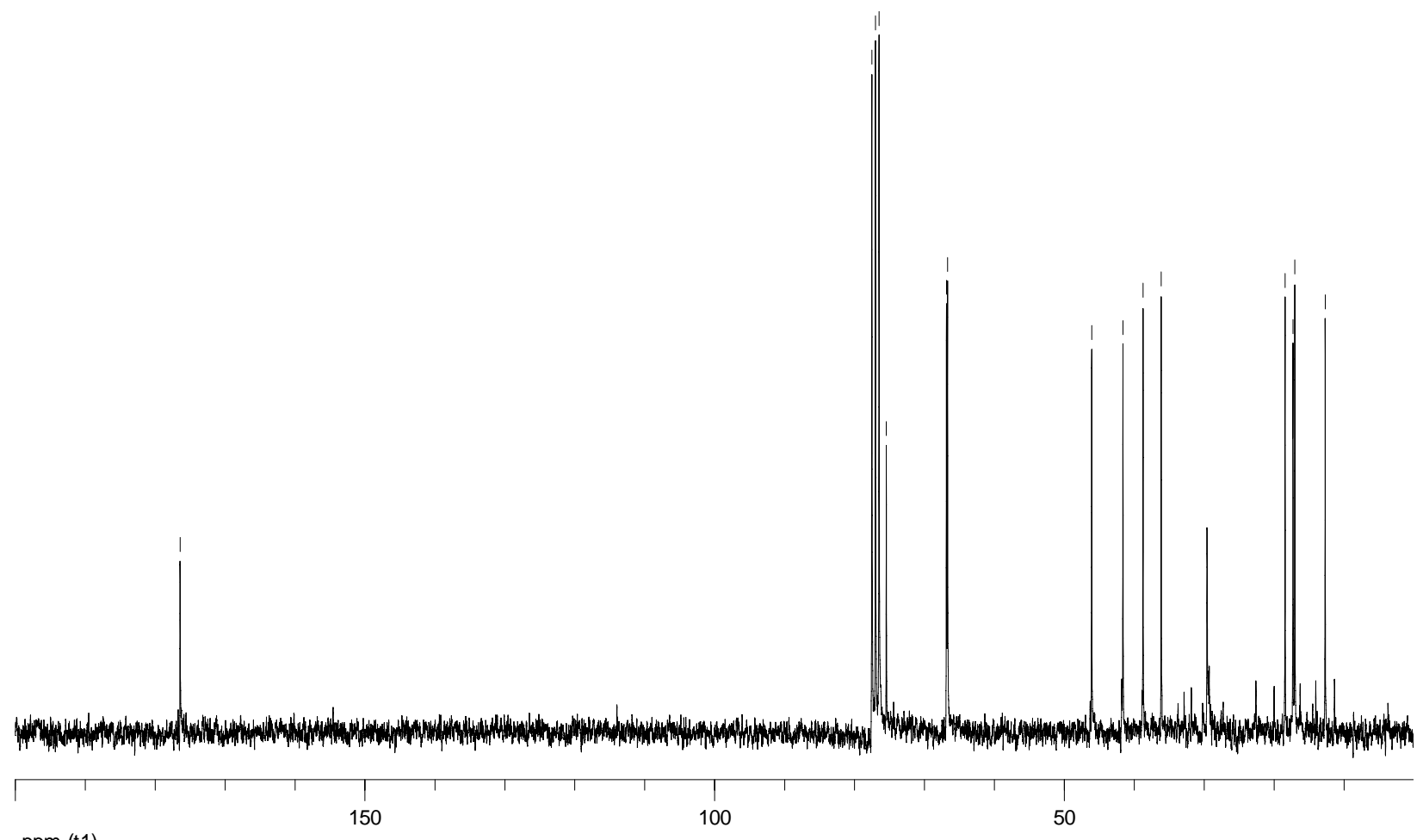

ppm (t1) 
<smiles>CC(C)C[C@](C)(O)C([14CH3])C(=O)N1CCOCC1</smiles>
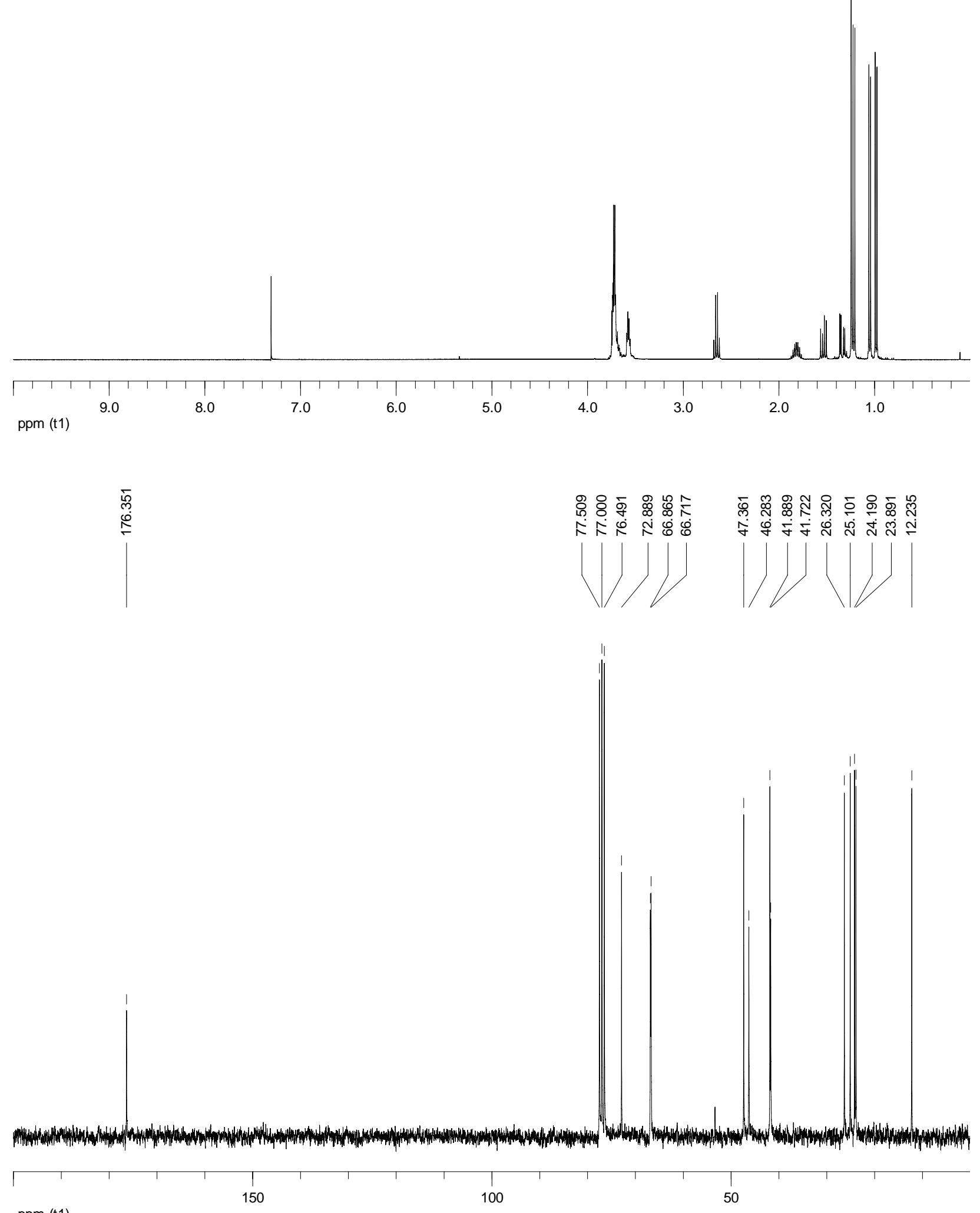

ppm (t1) 

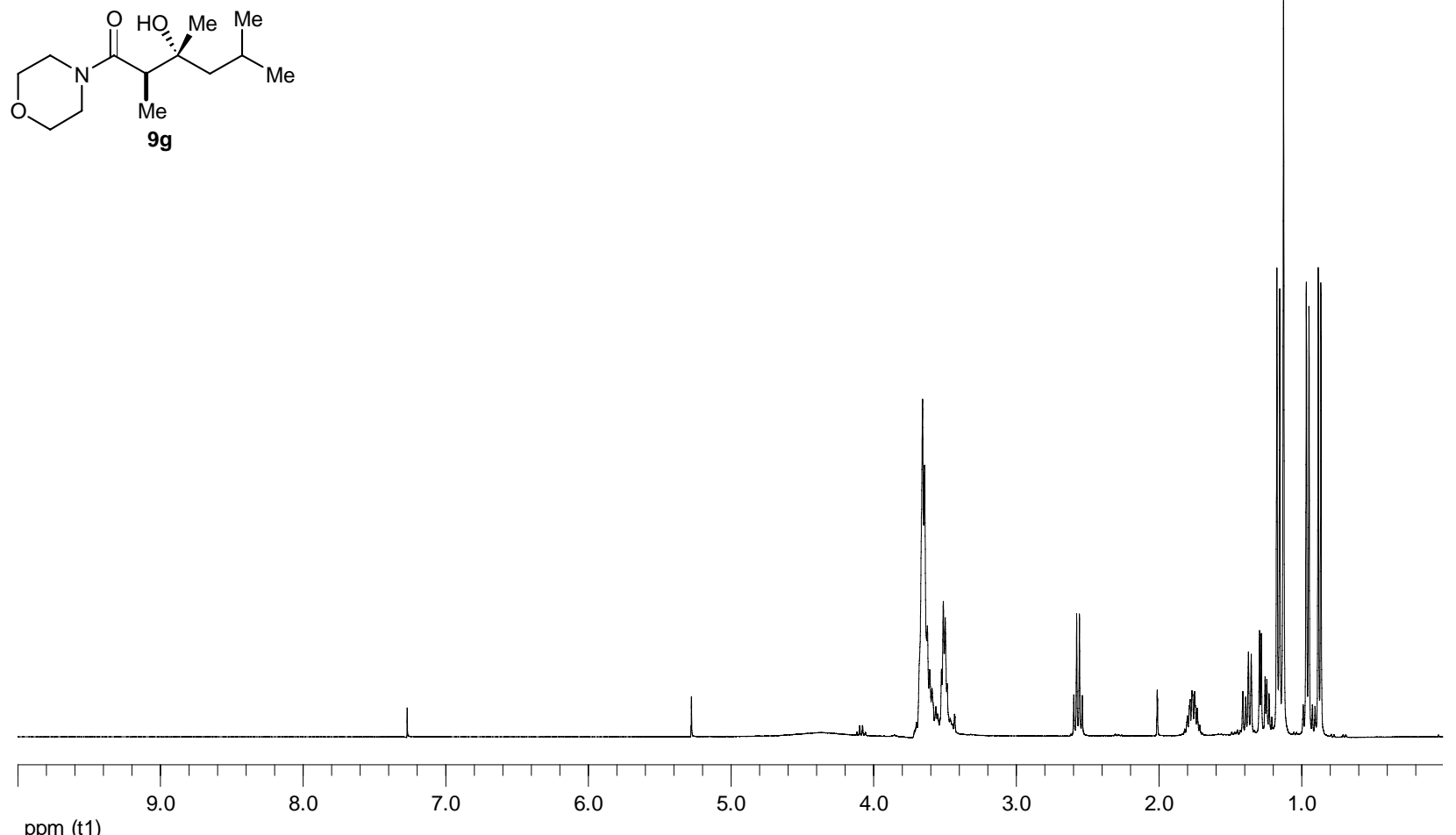

ppm (t1)

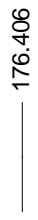

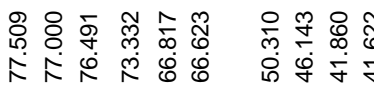

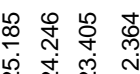
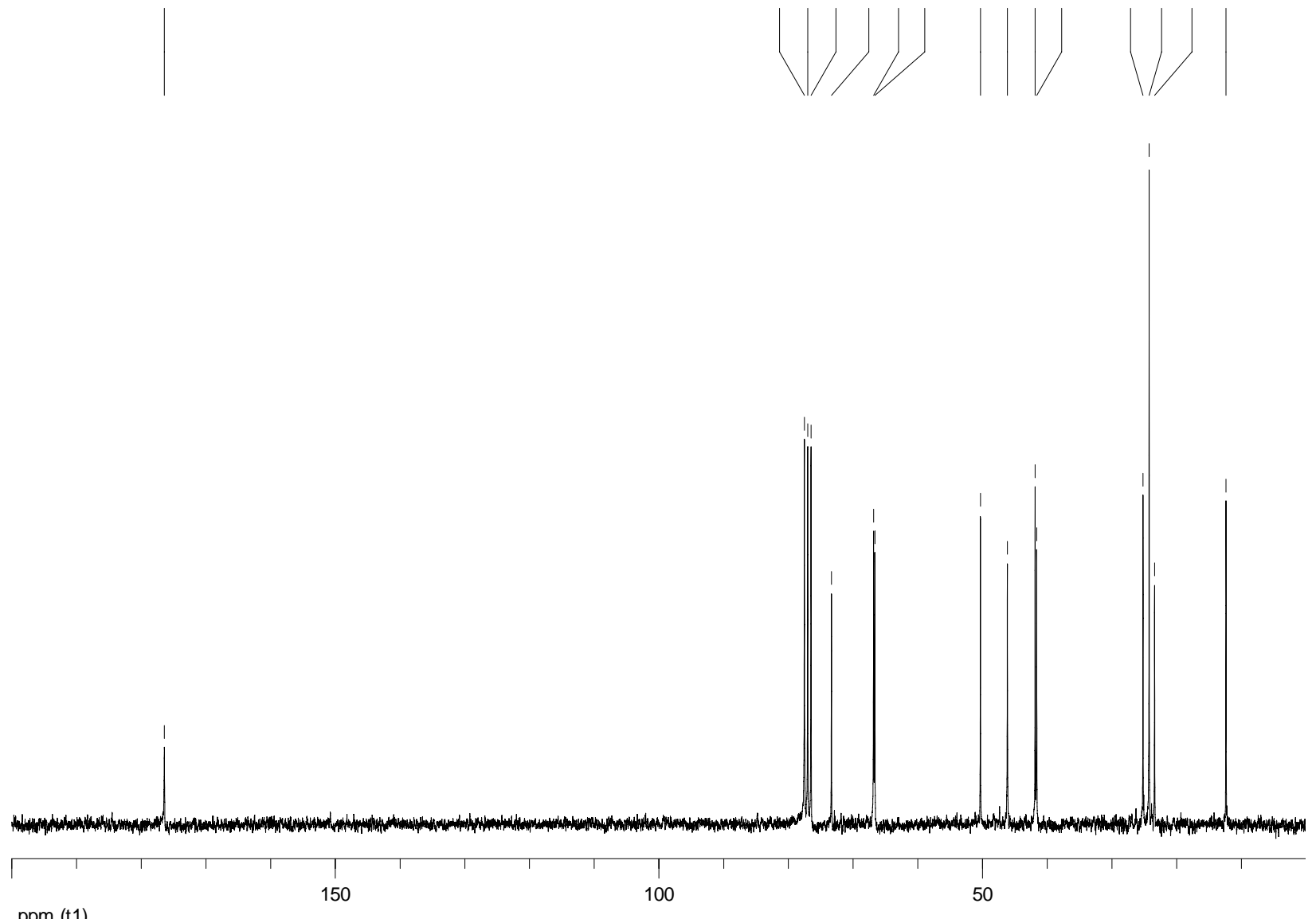

ppm (t1) 


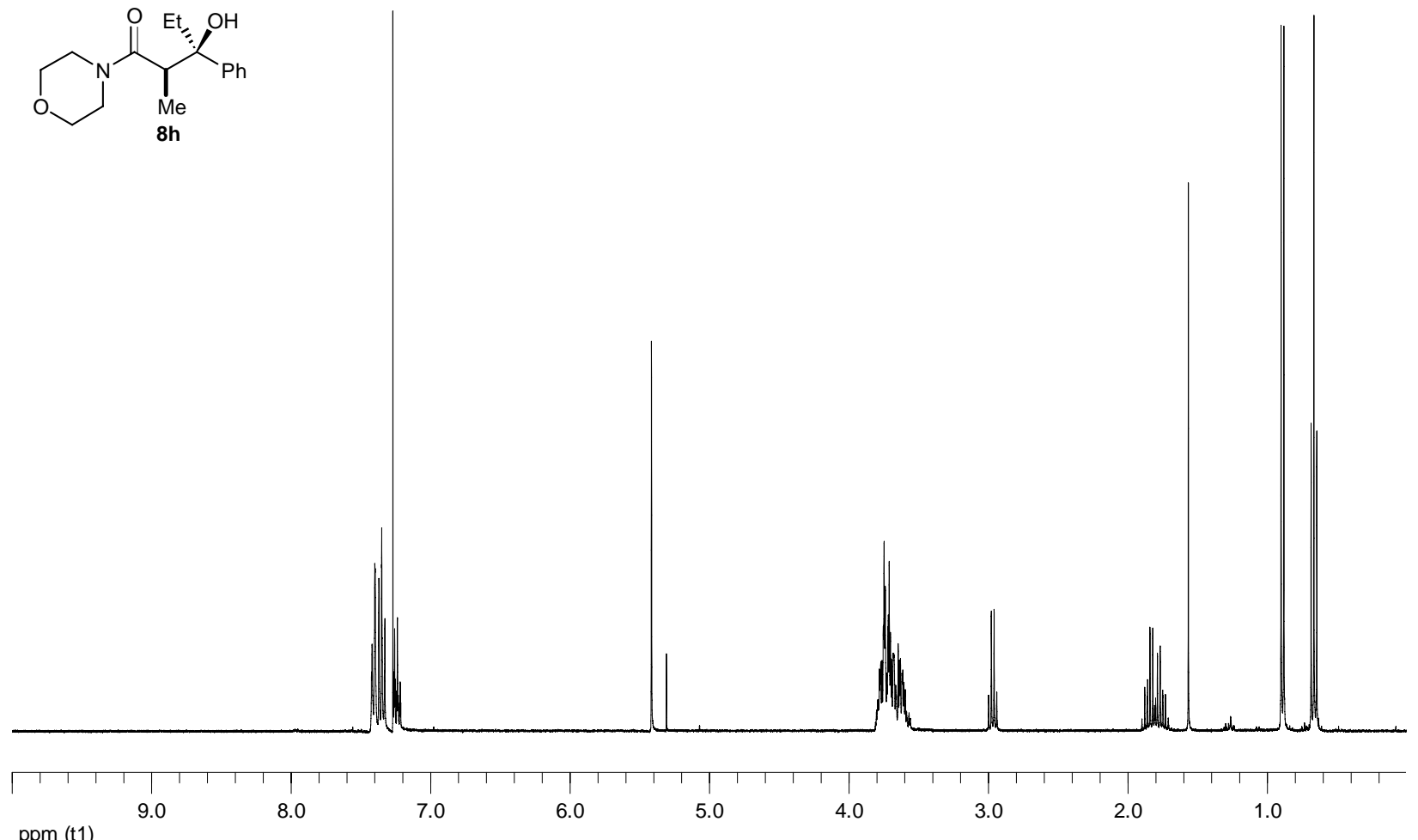

ppm (t1)

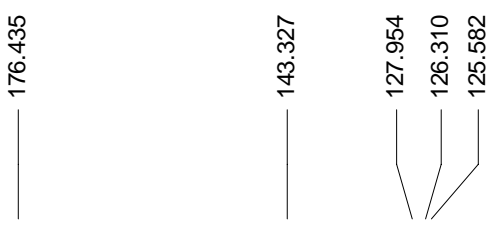
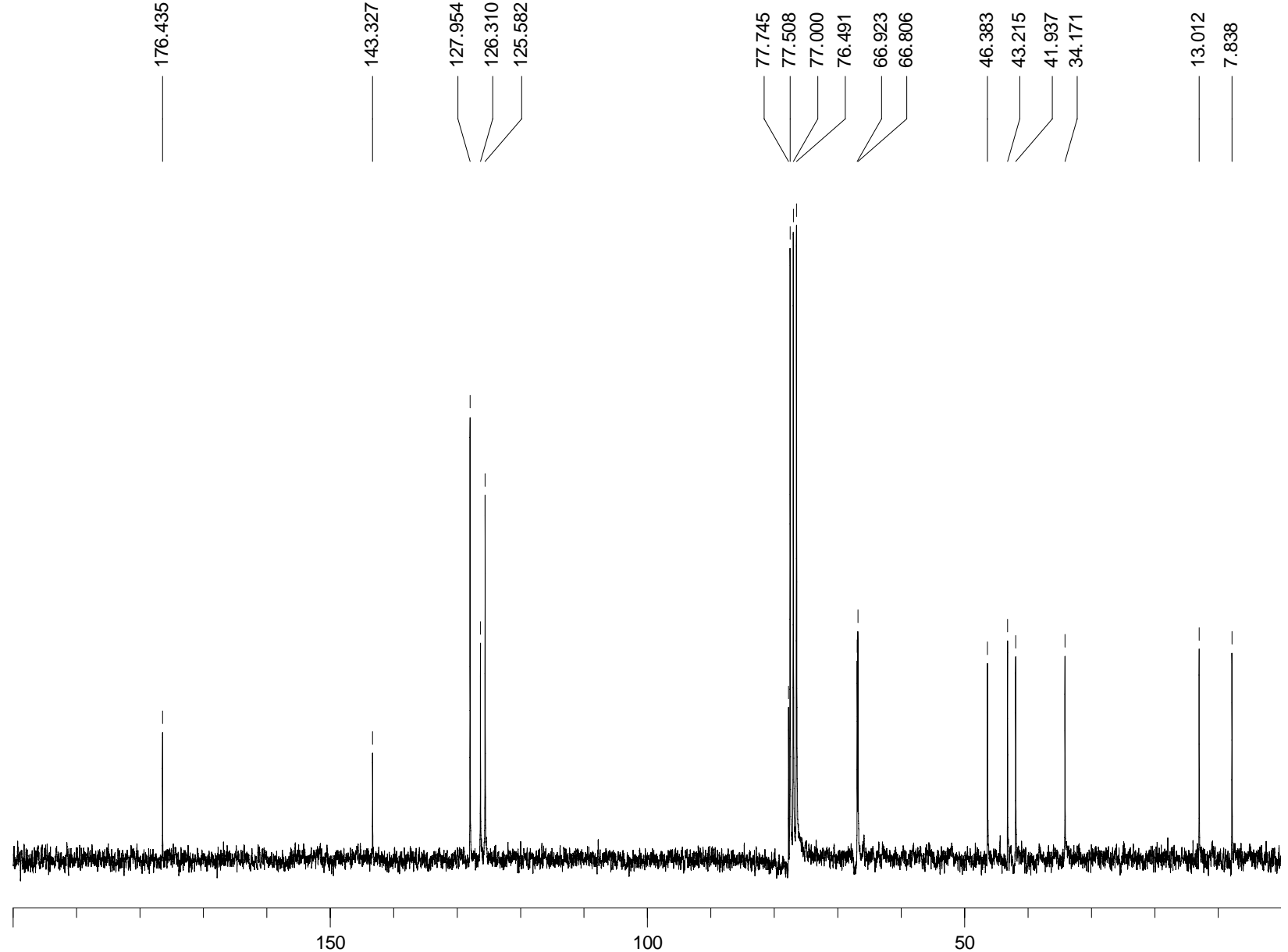

ppm (f1) 

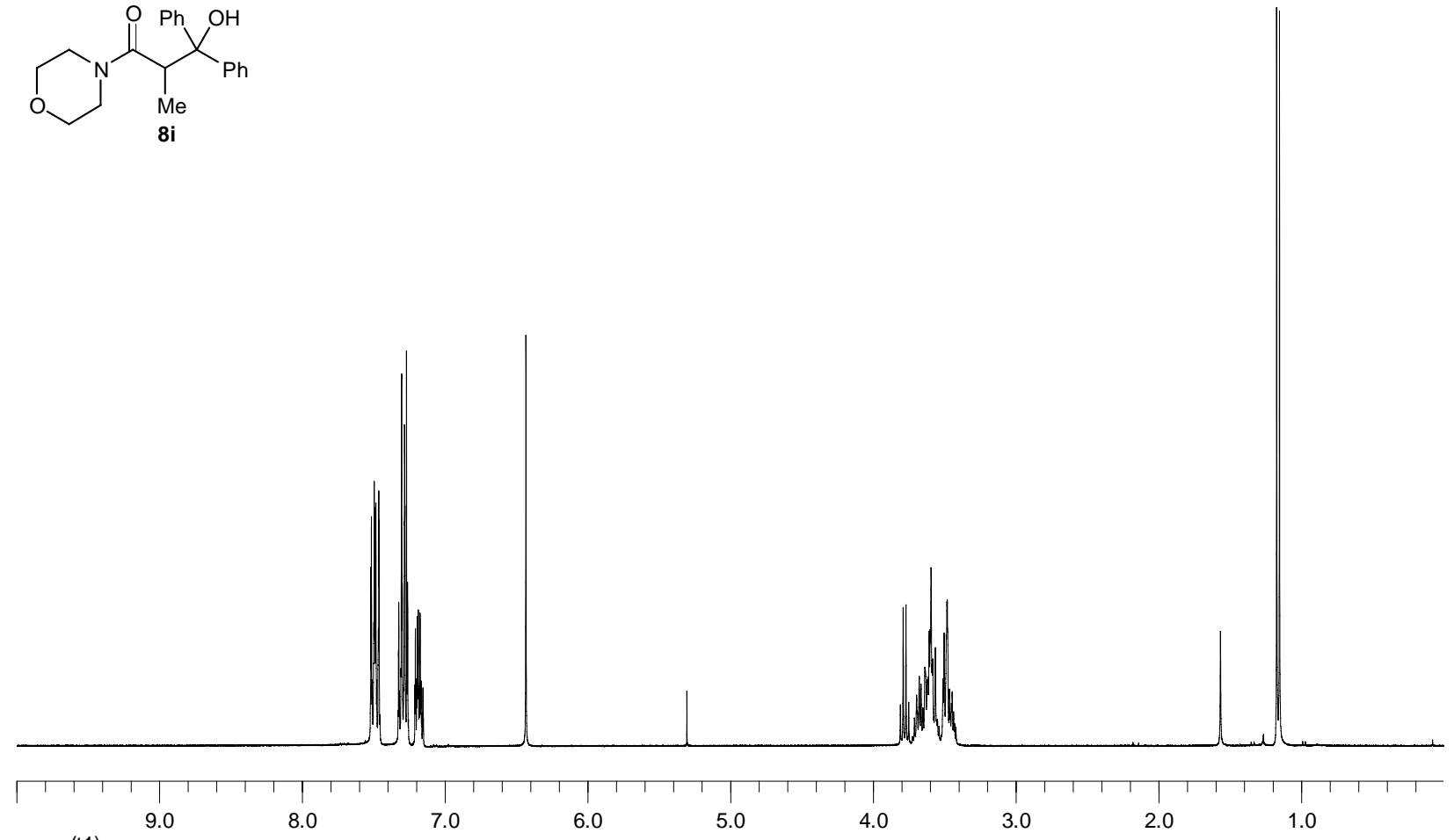

ppm (t1)
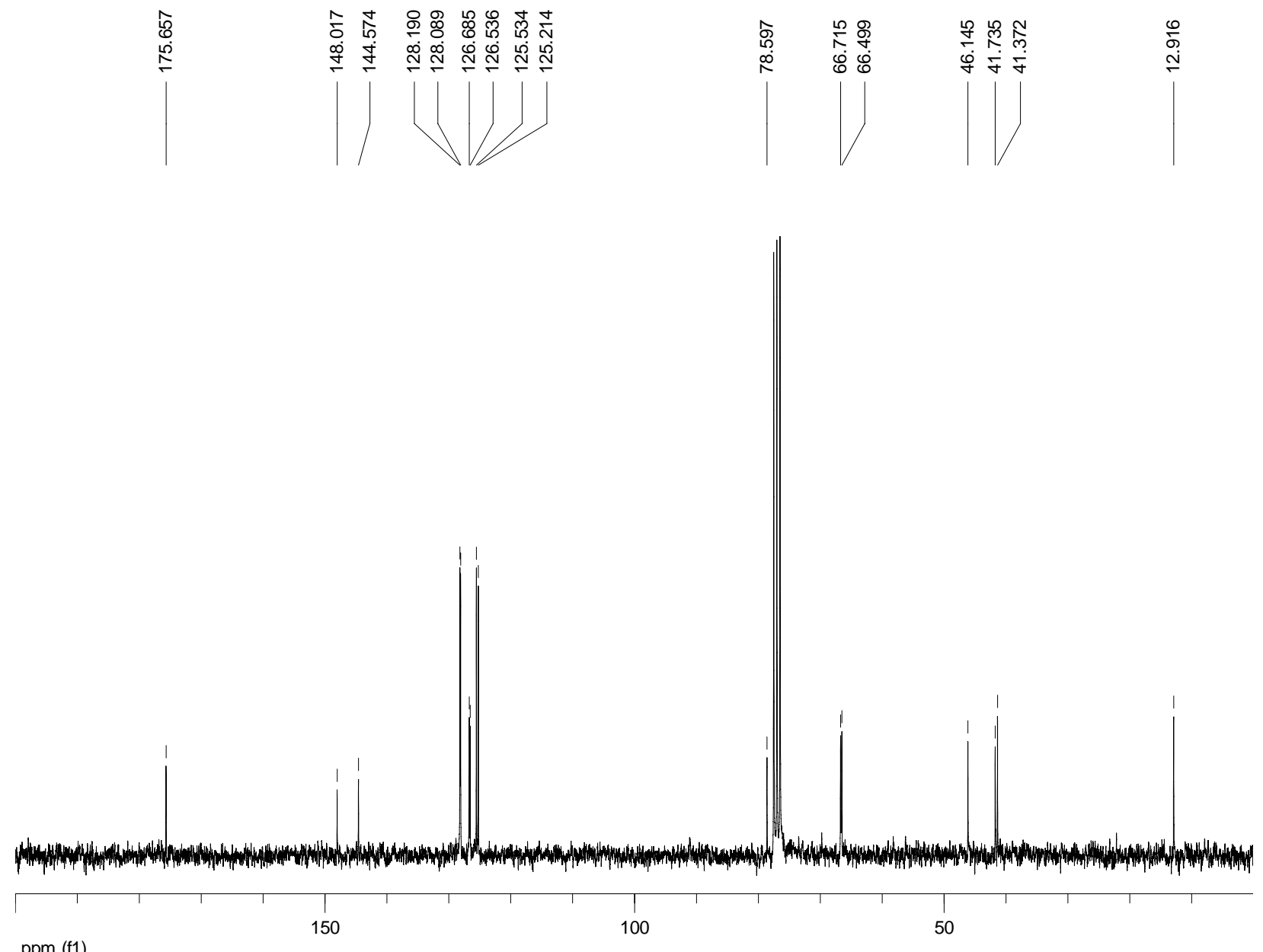

ppm (f1) 
<smiles>CCC(C(=O)N1CCOCC1)C(C)(O)c1ccccc1</smiles>

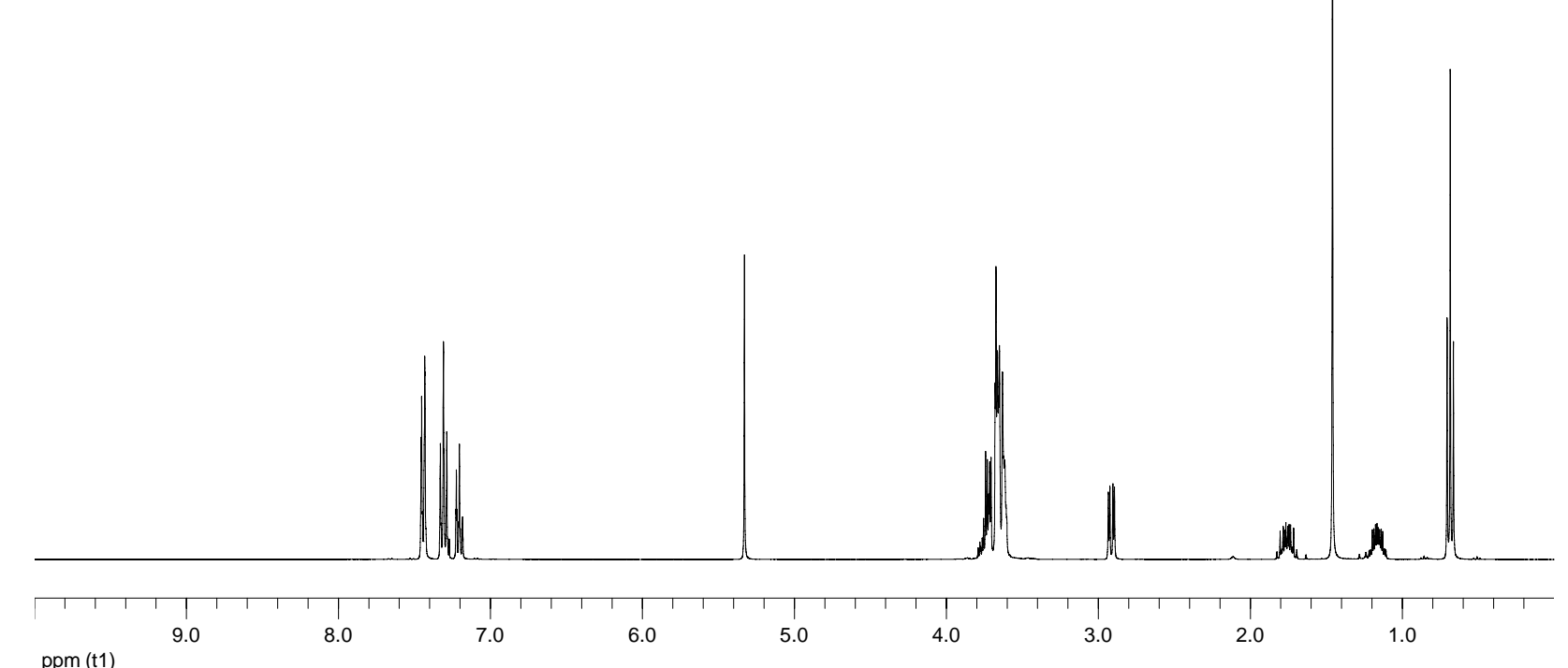

ppm (t1)
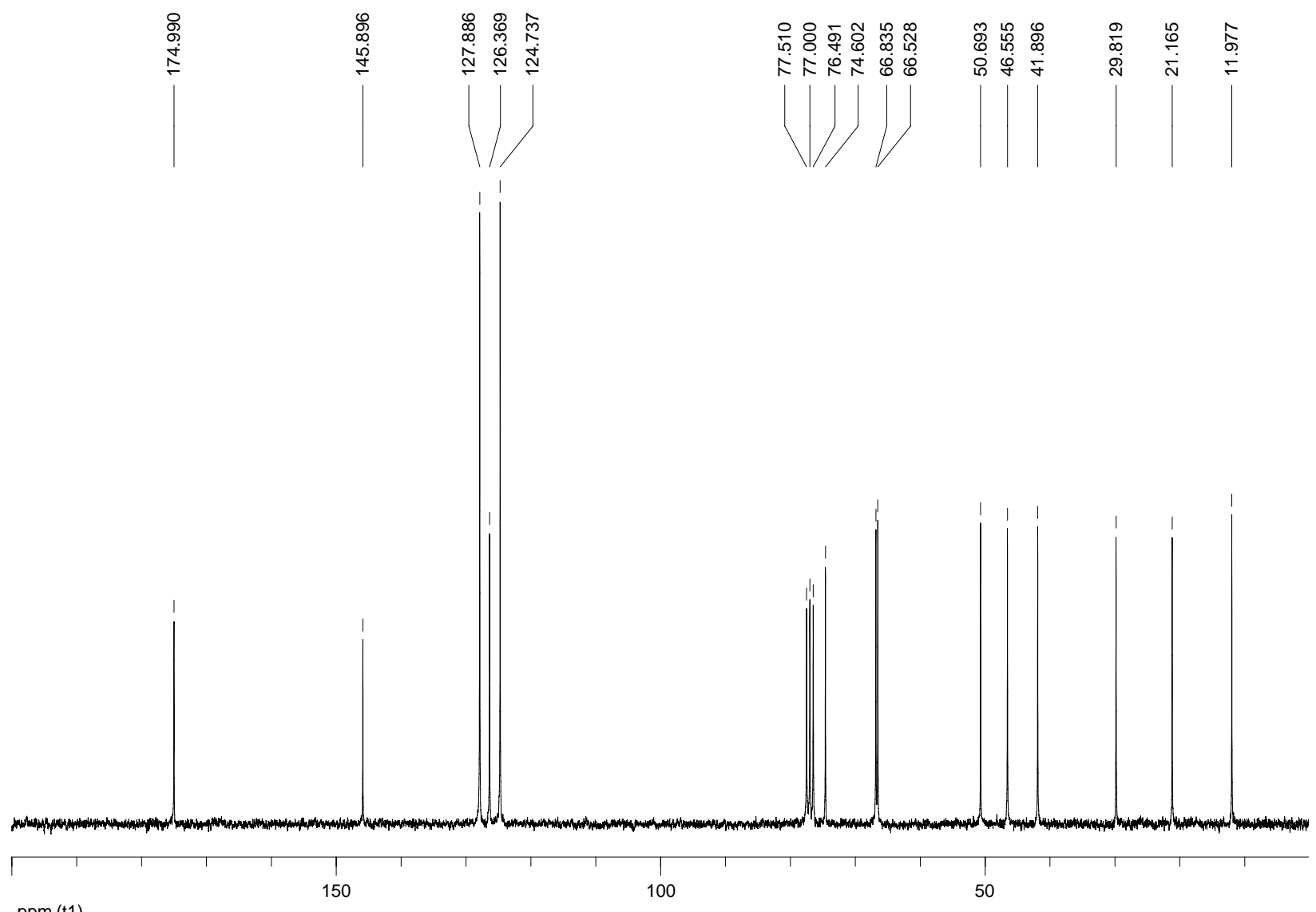

ppm (t1) 
Supporting Information

S46
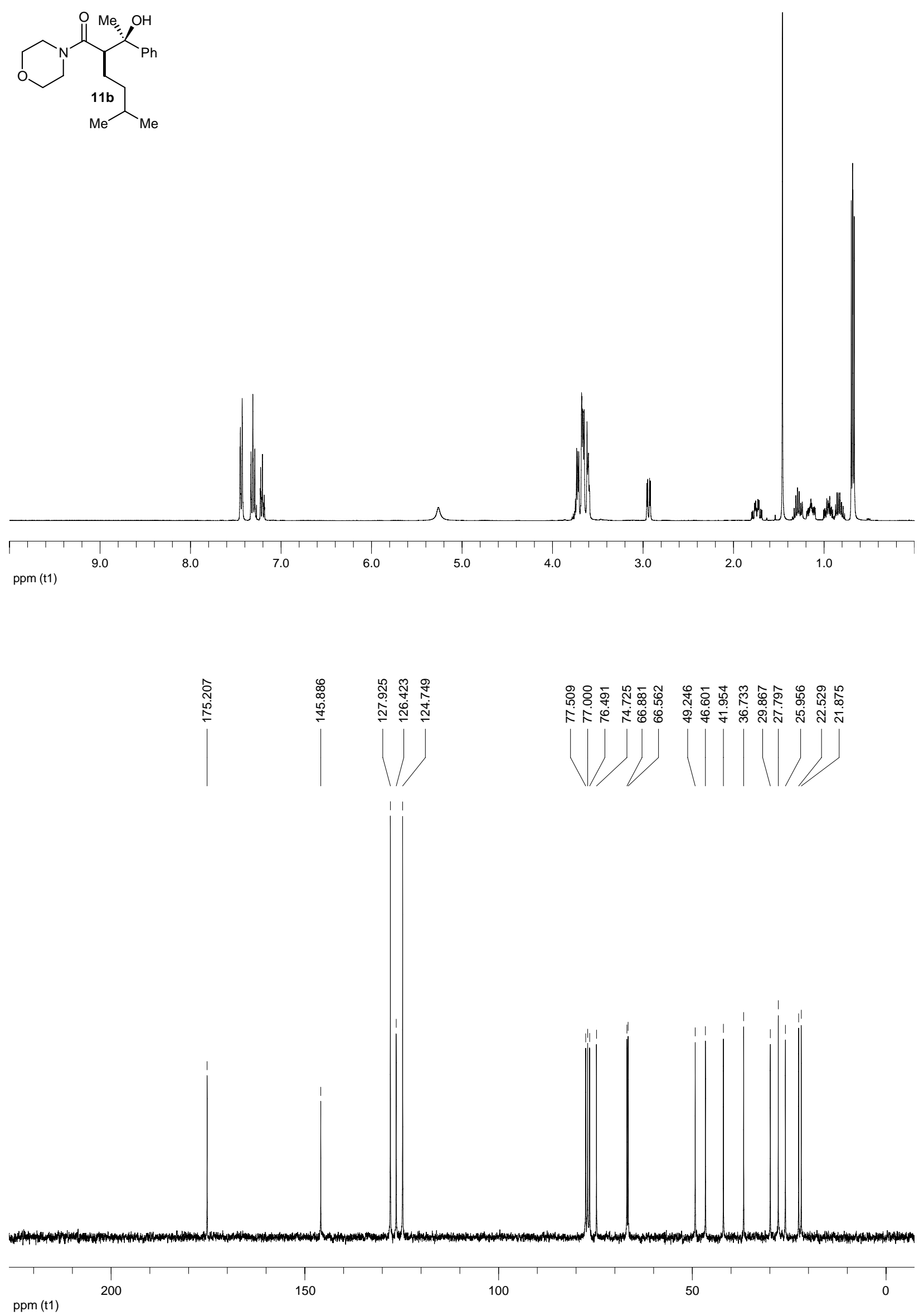
<smiles>CC(CCCc1ccccc1)(c1ccccc1)C(C)(O)C(CCCc1ccccc1)C(=O)N1CCOCC1</smiles>
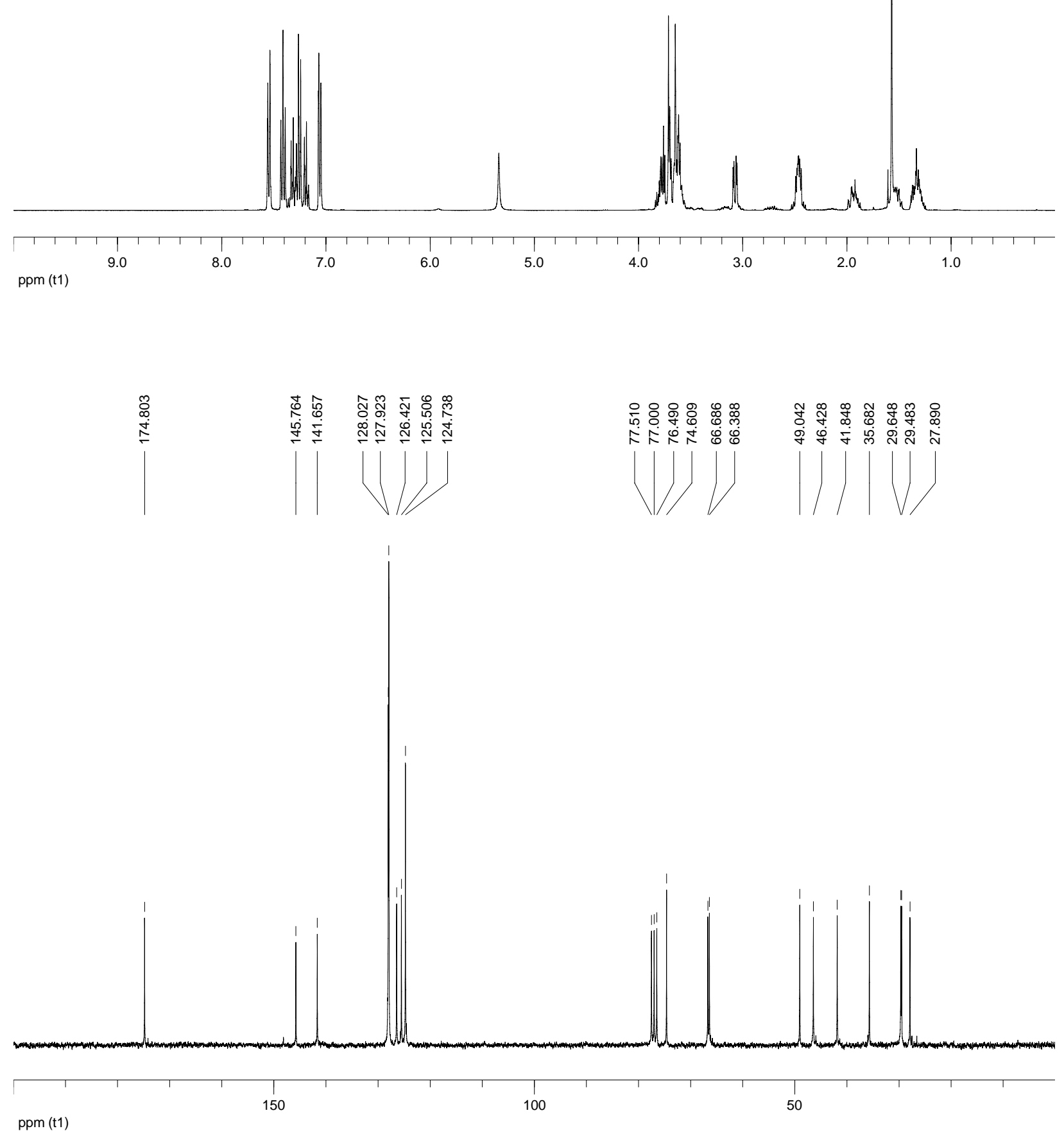

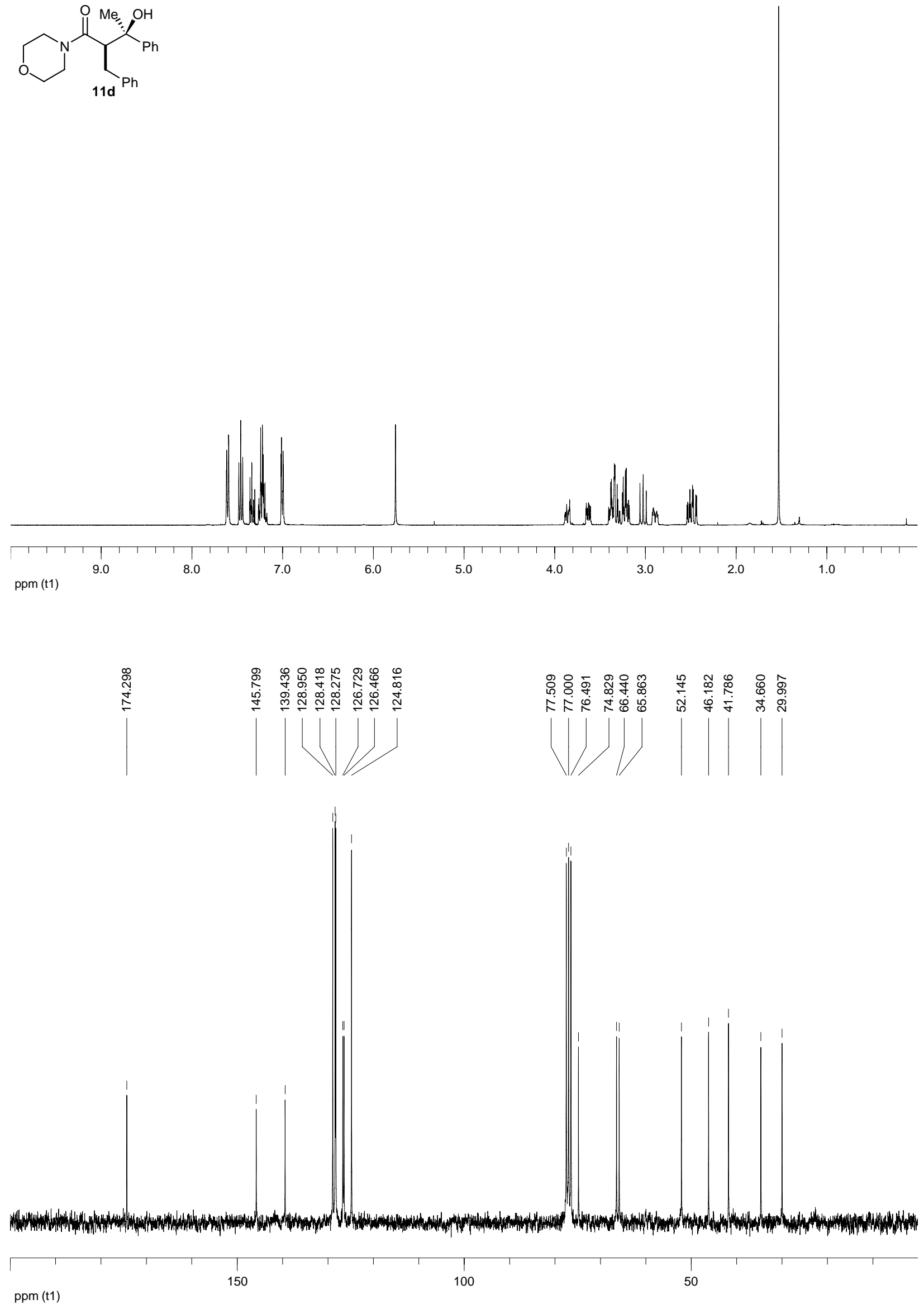
Supporting Information

S49
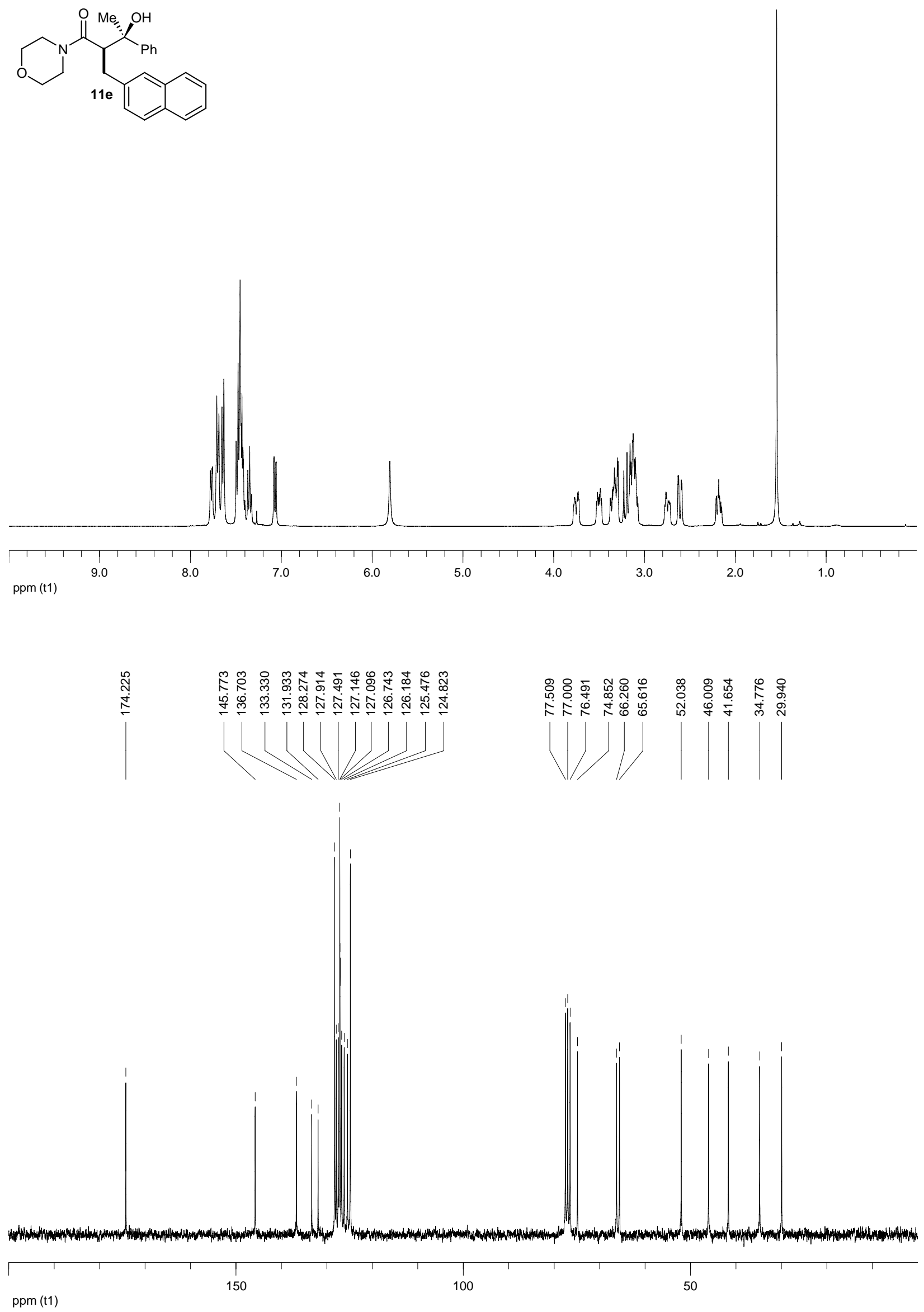

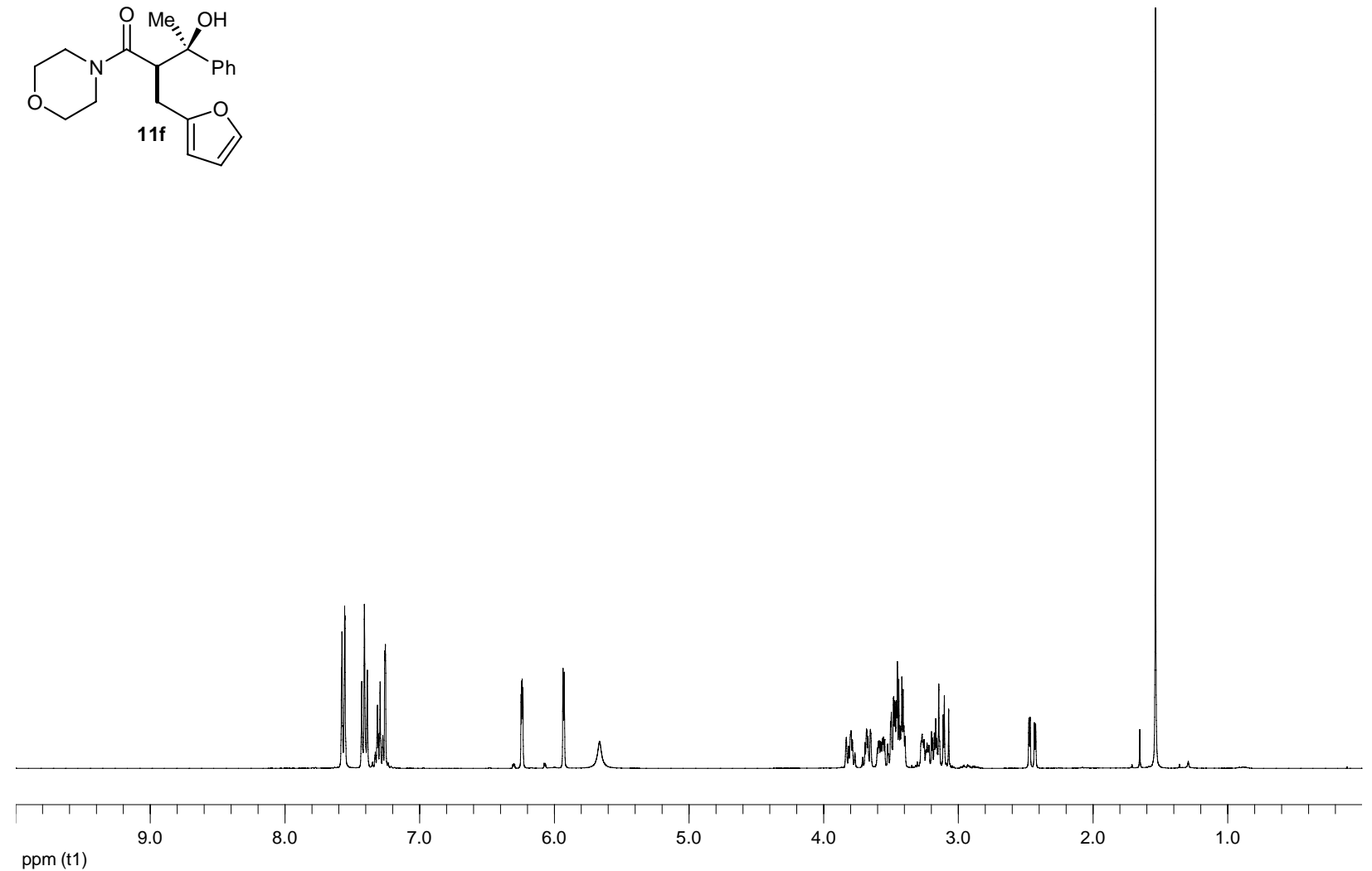

ppm (t1)
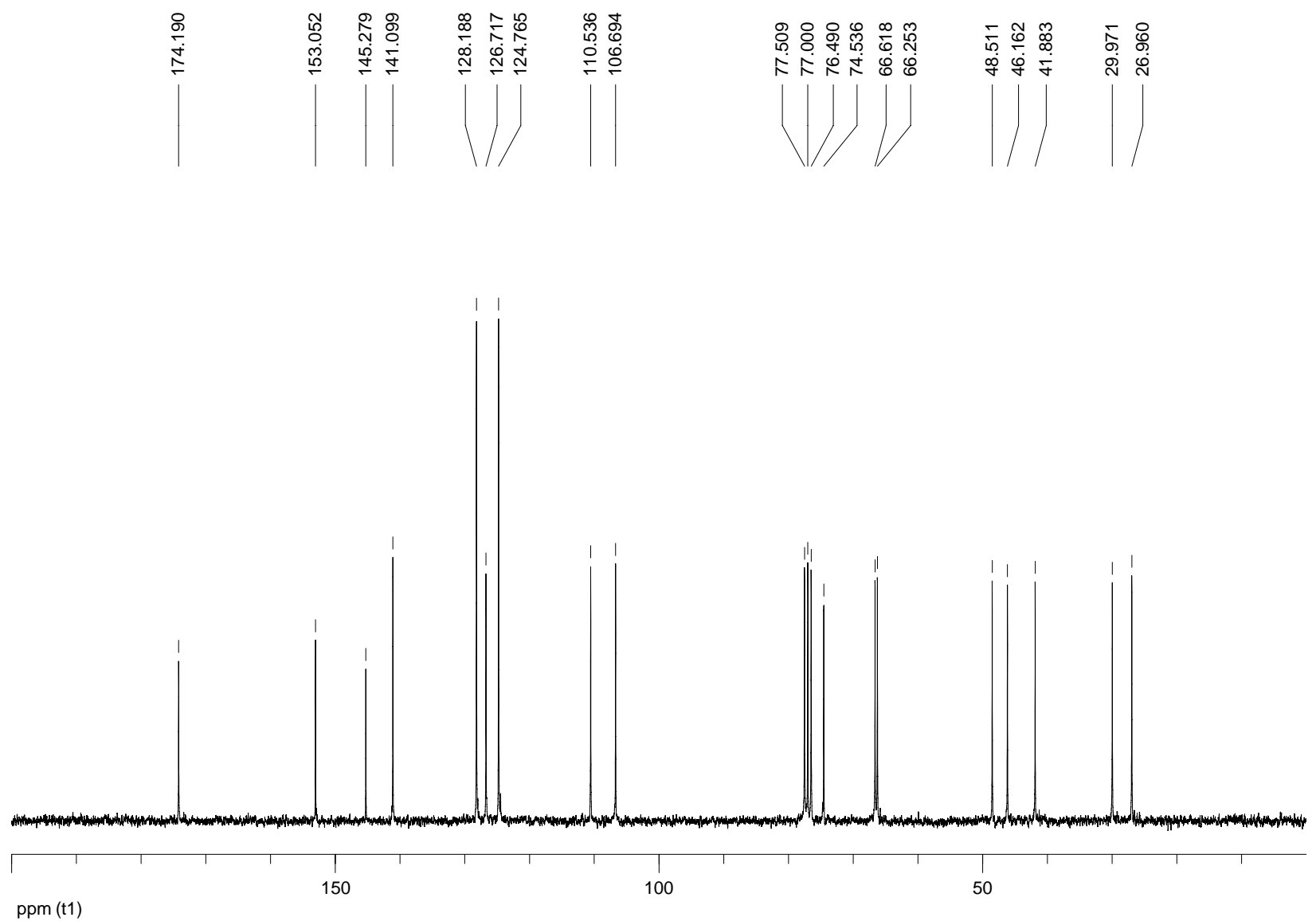
<smiles>[Y16]C1(C(C[14CH3])C(=O)N2CCOCC2)CCC(=O)O1</smiles>
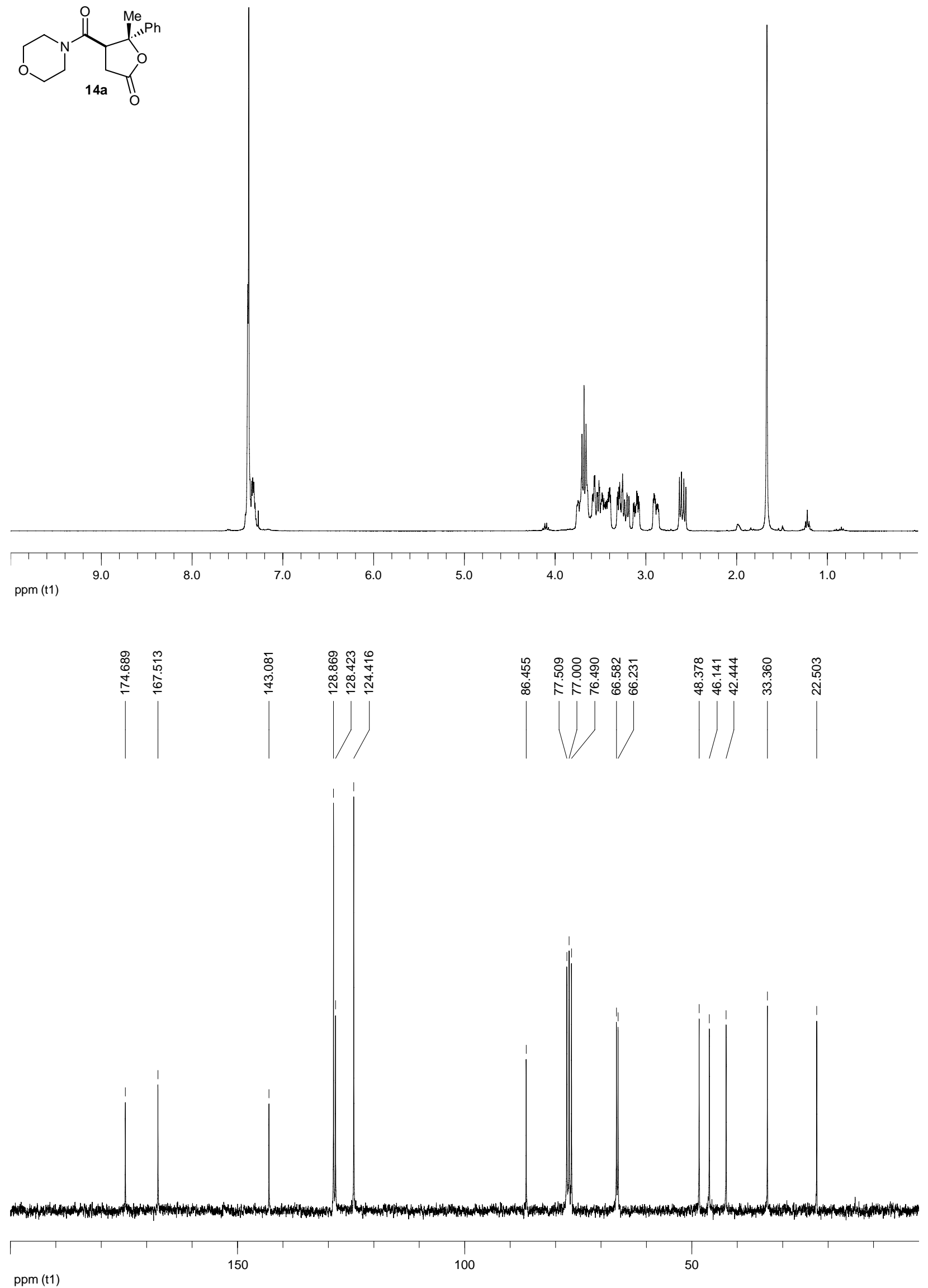
<smiles>COc1ccc([C@@]2(C)OC(=O)CC2C(=O)N2CCOCC2)cc1</smiles>
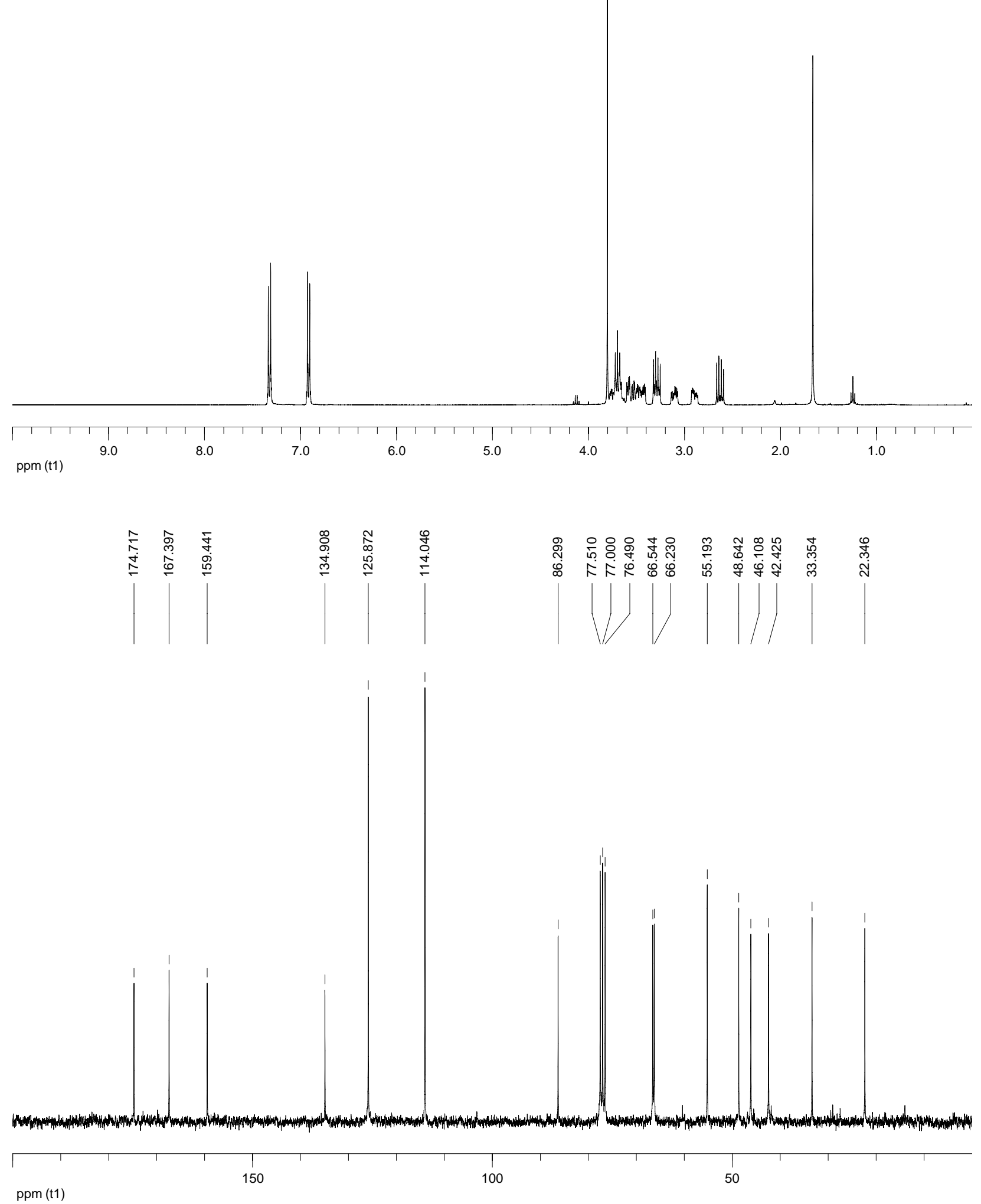
<smiles>C[C@@]1(c2ccc3ccccc3c2)OC(=O)CC1C(=O)N1CCOCC1</smiles>
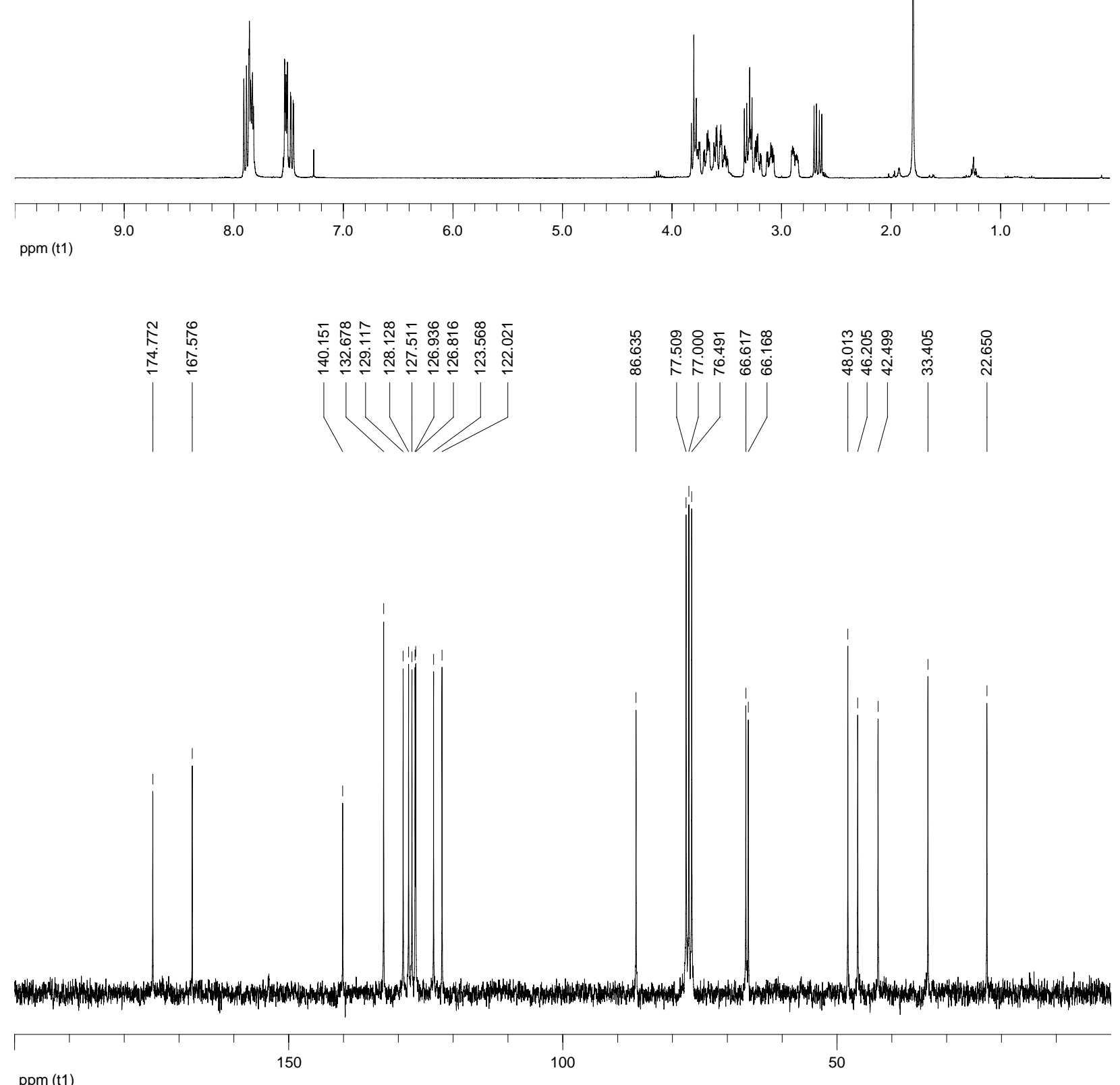

ppm (t1) 